\title{
Synthesis of Exclusively 4-Substituted $\beta$-Lactams through the Kinugasa Reaction Utilizing Calcium Carbide
}

\author{
Abolfazl Hosseini and Peter R. Schreiner* \\ Institute of Organic Chemistry, Justus Liebig University, Heinrich-Buff-Ring 17, 35392 Giessen, \\ Germany
}

\section{Supporting Information}

\section{Table of Contents}

General Information

Table S1

Table S2

Figure S1

Figure S2

General procedure for the preparation of nitrones $1 \mathrm{a}-\mathbf{1 k}, \mathbf{1 n}, \mathbf{1 0}$

Preparation of nitrone 11

Preparation of nitrone 1p

Typical procedure for the preparation of $\beta$-lactam 2a 
General Information: All chemicals were purchased from Aldrich, Alfa Aesar and Acros Organics in reagent grade or better quality and used without further purification. All reactions were carried out under argon or nitrogen atmosphere in a Schlenk tube. All solvents were dried before use. Analytical thin-layer chromatography (TLC) was performed on plastic-backed silica gel 60 coated with a fluorescence indicator. Visualization of TLC plate was performed by UV ( $254 \mathrm{~nm}$ ) or phosphomolybdic acid stain. Flash column chromatography was conducted using Merck silica gel $60(0.040-0.063 \mathrm{~mm}) .{ }^{1} \mathrm{H}$ and ${ }^{13} \mathrm{C}$ spectra were measured with Bruker spectrometer Avance II $400 \mathrm{MHz}$ (AV 400) and Avance III $600 \mathrm{MHz}$ (AV 600), using TMS as the internal standard. Chemical shifts are reported in parts per million (ppm). The progress of reactions was monitored by GC-MS analyses with a Quadrupol-MS HP MSD 5971(EI) and HP 5890A GC equipped with a J \& W Scientific fused silica GC column $(30 \mathrm{~m} \times 0.250 \mathrm{~mm}, 0.25$ micron DB-5MS stationary phase: $5 \%$ phenyl and 95\% methyl silicone) using He (4.6 grade) as carrier gas; T-program standard $60-250{ }^{\circ} \mathrm{C}\left(15{ }^{\circ} \mathrm{C} / \mathrm{min}\right.$ heating rate $)$, injector and transfer line $250{ }^{\circ} \mathrm{C}$. Calcium carbide (Acros Organics, 97\%) was powdered using mortar and pestle. $\mathrm{TBAF} \cdot 3 \mathrm{H}_{2} \mathrm{O}$ was used as $0.5 \mathrm{M}$ stock solution. Nitrones $\mathbf{1} \mathbf{m},{ }^{1} \mathbf{1 q},{ }^{2} \mathbf{1 r}^{3}$ and $\mathbf{1} \mathbf{s}^{4}$ were prepared according to the literature procedures. 
Table S1. Influence of main factor variations on the Kinugasa reaction.

\begin{tabular}{|c|c|c|c|c|c|c|c|c|}
\hline \multirow[b]{2}{*}{ No } & \multirow[b]{2}{*}{ Ligand (equiv) } & \multirow[b]{2}{*}{$\mathrm{CaC}_{2}$ (equiv) } & \multirow[b]{2}{*}{$\mathrm{H}_{2} \mathrm{O}$ (equiv) } & \multirow[b]{2}{*}{$\boldsymbol{T}^{\circ} \mathbf{C}$} & \multirow[b]{2}{*}{ Solvent } & \multirow[b]{2}{*}{$\mathrm{CuCl}$ (equiv) } & \multicolumn{2}{|c|}{ Yield (\%) $)^{a, b}$} \\
\hline & & & & & & & $2 a$ & o) \\
\hline 1 & NMI (6) & 1.3 & 5.6 & 0 & $\mathrm{DME}^{c}$ & 1 & 52 & 32 \\
\hline 2 & NMI (6) & 1.3 & 5.6 & 0 & $\mathrm{DME}^{d}$ & 1 & 49 & 32 \\
\hline $3^{e}$ & NMI (6) & 1.3 & 5.6 & 0 & DME & 1 & 26 & 23 \\
\hline 4 & DMAP (2) & 1.3 & 5.6 & 0 & DME & 1 & 21 & 30 \\
\hline 5 & NMI (2) & 1.3 & 5.6 & 0 & DME & 1 & 33 & 45 \\
\hline 6 & NMI (4) & 1.3 & 5.6 & 0 & DME & 1 & 48 & 45 \\
\hline 7 & NMI (10) & 1.3 & 5.6 & 0 & DME & 1 & 42 & 33 \\
\hline $8^{f}$ & NMI (6) & 1.3 & 5.6 & 0 & DME & 1 & 33 & 41 \\
\hline 9 & NMI (6) & 1.3 & 4.45 & 0 & DME & 1 & 53 & 33 \\
\hline 10 & NMI (6) & 1.3 & 5.6 & 0 & $\mathrm{MeCN}$ & 1 & 42 & 41 \\
\hline 11 & NMI (6) & 1.3 & 5.6 & 0 & $\mathrm{Et}_{2} \mathrm{O}$ & 1 & 41 & 39 \\
\hline 12 & NMI (6) & 1.3 & 5.6 & 0 & EtOAc & 1 & 25 & 21 \\
\hline 13 & NMI (6) & 1.3 & 5.6 & 0 & $\mathrm{CH}_{2} \mathrm{Cl}_{2}$ & 1 & 36 & 38 \\
\hline 14 & NMI (6) & 1.3 & 4.45 & 0 & DME & 0.5 & 32 & 22 \\
\hline $15^{g}$ & NMI (6) & 1.3 & 5 & 0 & DME & 1 & 29 & 20 \\
\hline 16 & NMI (6) & 1.3 & 4.45 & RT & DME & 1 & 36 & 40 \\
\hline 17 & NMI (6) & 1.8 & 5 & -20 & DME & 0.5 & (57) & (36) \\
\hline 18 & NMI (6) & 1.8 & 5 & -30 & DME & 0.5 & (58) & (27) \\
\hline 19 & NMI (6) & 1.3 & 5.6 & 0 & THF & 1 & (51) & (24) \\
\hline $20^{h}$ & NMI (6) & 1.3 & 5.6 & 0 & THF & 1 & 12 & 33 \\
\hline 21 & NMI (6) & 1.8 & 5 & -20 & THF & 0.5 & (64) & (27) \\
\hline
\end{tabular}

${ }^{a}$ NMR yield. ${ }^{b}$ Numbers in parenthesis show isolated yield of product. ${ }^{c}$ Both deoxygenation with $\mathrm{Ar}$ and $\mathrm{N}_{2}$ afforded the same result. ${ }^{d}$ Freeze-pump-thaw technique. ${ }^{e}$ TBABr was used instead of TBAF. ${ }^{f} \mathrm{CuI}$ was used instead of $\mathrm{CuCl} .{ }^{g} 0.5$ equiv of TBAF was used. ${ }^{h}$ Reaction in the absence of TBAF. 
Table S2. Reaction conditions obtained by DoE analysis and the resulting yields.

\begin{tabular}{|c|c|c|c|c|c|c|c|}
\hline Entry & NMI & $\mathrm{H}_{2} \mathrm{O}$ & $\mathrm{CaC}_{2}$ & Temp. $\left({ }^{\circ} \mathrm{C}\right)$ & $\mathrm{CuCl}$ & TBAF & Yield of $2 \mathrm{a}^{a}$ \\
\hline 1 & 6 & 5.05 & 1.7 & 20 & 0.63 & 0.3 & 51 \\
\hline 2 & 9 & 5.05 & 1.7 & 20 & 0.63 & 0.3 & 56 \\
\hline 3 & 9 & 4.5 & 2.4 & 0 & 0.63 & 0.1 & 73 \\
\hline 4 & 9 & 5.05 & 2.4 & 0 & 1 & 0.3 & 75 \\
\hline 5 & 6 & 5.6 & 2.4 & 20 & 1 & 0.1 & 47 \\
\hline 6 & 9 & 5.6 & 1 & -20 & 0.26 & 0.5 & 34 \\
\hline 7 & 6 & 4.5 & 1.7 & 0 & 0.26 & 0.5 & 25 \\
\hline 8 & 3 & 4.5 & 1 & 20 & 0.26 & 0.1 & 22 \\
\hline 9 & 9 & 5.6 & 2.4 & -20 & 0.63 & 0.1 & 59 \\
\hline 10 & 9 & 5.6 & 1 & 20 & 1 & 0.5 & 27 \\
\hline 11 & 9 & 5.6 & 2.4 & 20 & 0.26 & 0.5 & 4 \\
\hline 12 & 6 & 4.5 & 2.4 & -20 & 0.26 & 0.3 & 59 \\
\hline 13 & 6 & 5.6 & 1 & -20 & 1 & 0.3 & 33 \\
\hline 14 & 9 & 4.5 & 1 & 20 & 0.26 & 0.5 & 6 \\
\hline 15 & 6 & 5.05 & 1 & 0 & 0.63 & 0.3 & 43 \\
\hline 16 & 6 & 5.05 & 1.7 & 0 & 0.63 & 0.1 & 58 \\
\hline 17 & 3 & 5.6 & 1 & 20 & 1 & 0.1 & 18 \\
\hline 18 & 9 & 5.6 & 1 & 20 & 0.26 & 0.1 & 37 \\
\hline 19 & 3 & 5.6 & 1.7 & -20 & 1 & 0.5 & 70 \\
\hline 20 & 9 & 4.5 & 1 & -20 & 0.26 & 0.1 & 63 \\
\hline 21 & 6 & 5.05 & 2.4 & -20 & 0.63 & 0.5 & 74 \\
\hline 22 & 3 & 5.6 & 1 & 20 & 0.26 & 0.5 & 5 \\
\hline 23 & 9 & 4.5 & 1.7 & 20 & 1 & 0.1 & 63 \\
\hline 24 & 9 & 5.05 & 1 & 0 & 1 & 0.1 & 48 \\
\hline 25 & 6 & 4.5 & 1 & 20 & 1 & 0.5 & 29 \\
\hline 26 & 3 & 5.6 & 2.4 & 0 & 0.63 & 0.3 & 79 \\
\hline 27 & 3 & 5.05 & 1.7 & 0 & 1 & 0.3 & 74 \\
\hline 28 & 3 & 4.5 & 1 & -20 & 0.63 & 0.5 & 43 \\
\hline 29 & 3 & 4.5 & 2.4 & 20 & 0.63 & 0.5 & 30 \\
\hline 30 & 3 & 5.05 & 2.4 & 20 & 0.26 & 0.1 & 15 \\
\hline 31 & 6 & 5.6 & 1.7 & 0 & 0.63 & 0.3 & 67 \\
\hline 32 & 3 & 5.6 & 1 & -20 & 0.26 & 0.1 & 37 \\
\hline 33 & 9 & 4.5 & 1.7 & -20 & 1 & 0.5 & 72 \\
\hline 34 & 3 & 4.5 & 2.4 & -20 & 1 & 0.1 & 28 \\
\hline 35 & 6 & 5.6 & 2.4 & 0 & 0.63 & 0.3 & 79 \\
\hline 36 & 6 & 5.6 & 2.4 & 0 & 0.86 & 0.3 & 79 \\
\hline 37 & 3 & 5.6 & 2.4 & 0 & 0.63 & 0.3 & 81 \\
\hline 38 & 6 & 5.05 & 1.7 & 0 & 0.63 & 0.3 & 69 \\
\hline 39 & 6 & 5.05 & 2.4 & -20 & 0.63 & 0.5 & 75 \\
\hline 40 & 6 & 5.6 & 1.7 & 0 & 0.63 & 0.3 & 67 \\
\hline 41 & 9 & 4.5 & 1.7 & -20 & 1 & 0.5 & 74 \\
\hline
\end{tabular}

${ }^{a}$ Yields were determined by NMR. 
Figure S1. Actual vs predicted values for the yield.

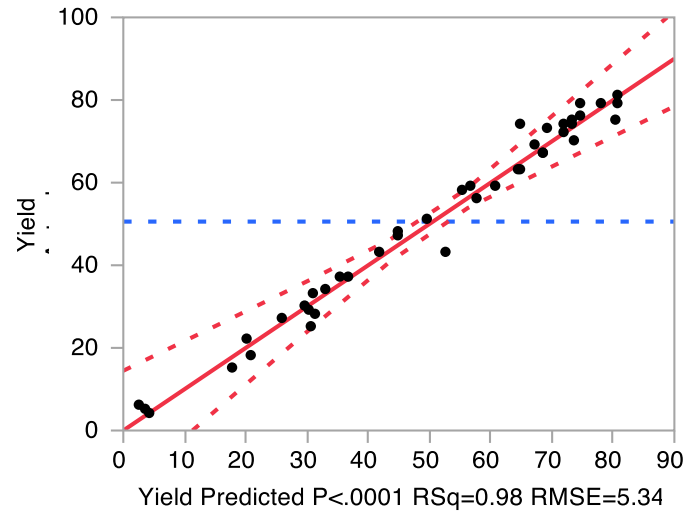

Figure S2. Prediction profiler for the lactam 2a based on the DoE analysis.

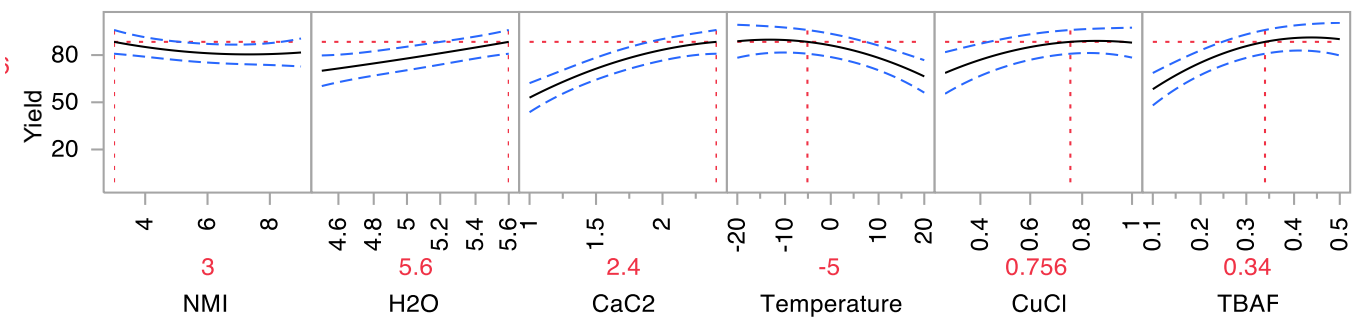




\section{General procedure for the preparation of nitrones $1 \mathrm{a}-1 \mathrm{k}, 1 \mathrm{n}$, and $1 \mathrm{o}$ :}

Nitroarene (47.1 mmol), aldehyde (47.1 mmol), $\mathrm{NH}_{4} \mathrm{Cl}(61.5 \mathrm{mmol}), \mathrm{EtOH}(50 \mathrm{~mL})$, and water $(50 \mathrm{~mL})$ were added to a $250 \mathrm{~mL}$ round-bottom flask. The reaction mixture was cooled to $0{ }^{\circ} \mathrm{C}$, and zinc $(94.5$ mmol) was added slowly over $2-3 \mathrm{~h}$. The suspension was then allowed to reach room temperature and stirred for $12 \mathrm{~h}$. The reaction mixture was filtered through a pad of celite and washed with $\mathrm{CH}_{2} \mathrm{Cl}_{2}$. The filtrate was extracted with $\mathrm{CH}_{2} \mathrm{Cl}_{2}$ and the combined organic layers were dried over $\mathrm{Na}_{2} \mathrm{SO}_{4}$, filtered and concentrated to give the crude nitrones. Pure nitrones were obtained by recrystallization from ethanol or column chromatography.

\section{Preparation of nitrone 11:}

2-Cyano-5-nitrobenzotrifluoride ( $0.5 \mathrm{~g}, 2.32 \mathrm{mmol})$, benzaldehyde $(0.3 \mathrm{~g}, 2.8 \mathrm{mmol}), \mathrm{NH}_{4} \mathrm{Cl}(0.16 \mathrm{~g}, 3$ $\mathrm{mmol})$, and water $(5 \mathrm{~mL})$ were added to a Schlenk tube. The reaction mixture was placed in an ultrasonic bath $(37 \mathrm{kHz})$ and warmed to $50^{\circ} \mathrm{C}$, and zinc $(0.25 \mathrm{~g}, 3.8 \mathrm{mmol})$ was added portionwise over $10 \mathrm{~min}$. The suspension was ultrasonicated for $30 \mathrm{~min}$. The reaction mixture was filtered through a pad of celite and washed with $\mathrm{CH}_{2} \mathrm{Cl}_{2}$. The filtrate was extracted with $\mathrm{CH}_{2} \mathrm{Cl}_{2}$ and the combined organic layers were dried over $\mathrm{Na}_{2} \mathrm{SO}_{4}$, filtered and concentrated to give the crude nitrone. The resultant crude nitrone was purified by flash column chromatography (silica gel, EtOAc/hexanes) to give the corresponding nitrone 11 ( $0.28 \mathrm{~g}, 42 \%$ yield) as a pale yellow solid. ${ }^{1} \mathrm{H}$ NMR $\left(400 \mathrm{MHz}, \mathrm{CDCl}_{3}\right) \delta 8.46-8.38(\mathrm{~m}, 2 \mathrm{H}), 8.32$ $(\mathrm{m}, 1 \mathrm{H}), 8.14(\mathrm{~m}, 1 \mathrm{H}), 8.03(\mathrm{~s}, 1 \mathrm{H}), 7.98(\mathrm{~m}, 1 \mathrm{H}), 7.60-7.47(\mathrm{~m}, 3 \mathrm{H}) .{ }^{13} \mathrm{C} \mathrm{NMR}\left(101 \mathrm{MHz}, \mathrm{CDCl}_{3}\right) \delta$ $151.4,135.9,134.3\left(\mathrm{q}, J_{\mathrm{C}-\mathrm{F}}=33.8 \mathrm{~Hz}\right), 132.3,129.7,129.6,128.9,125.2,121.7\left(\mathrm{q}, J_{\mathrm{C}-\mathrm{F}}=274.4 \mathrm{~Hz}\right)$, $120.8\left(\mathrm{q}, J_{\mathrm{C}-\mathrm{F}}=4.9 \mathrm{~Hz}\right), 114.4,111.3\left(\mathrm{q}, J_{\mathrm{C}-\mathrm{F}}=1.8 \mathrm{~Hz}\right)$. HRMS exact mass calculated for $[\mathrm{M}+\mathrm{Na}]^{+}$ $\left(\mathrm{C}_{15} \mathrm{H}_{9} \mathrm{~F}_{3} \mathrm{~N}_{2} \mathrm{ONa}^{+}\right): m / z$ 313.0558, found $m / z 313.0556$.

\section{Preparation of nitrone 1p:}

An oven-dried Schlenk tube equipped with a magnetic stir bar under argon atmosphere was charged with $N$-phenylhydroxylamine $(0.12 \mathrm{~g}, 1.1 \mathrm{mmol})$ and $\mathrm{Et}_{2} \mathrm{O}(1.5 \mathrm{~mL})$. The reaction mixture was cooled $-20{ }^{\circ} \mathrm{C}$, and freshly distilled acetaldehyde $(0.07 \mathrm{~g}, 1.6 \mathrm{mmol})$ was subsequently added. After $10 \mathrm{~min}$ calcium oxide $(0.2 \mathrm{~g}, 3.6 \mathrm{mmol})$ was added and the reaction mixture was stirred for further $20 \mathrm{~min}$. The reaction mixture was filtered and washed with $\mathrm{Et}_{2} \mathrm{O}(1 \mathrm{~mL})$. The filtrate was placed in an ice bath and the ether was evaporated under vacuum. The resulting crude product was used without further purification. The amounts of nitrone was calculated by NMR using 1,4-dinitrobenzene as internal standard. ${ }^{1} \mathrm{H}$ NMR $\left(400 \mathrm{MHz}, \mathrm{CDCl}_{3}\right) \delta 7.64-7.59(\mathrm{~m}, 2 \mathrm{H}), 7.41-7.37(\mathrm{~m}, 3 \mathrm{H}), 7.31(\mathrm{q}, J=6.0 \mathrm{~Hz}$, $1 \mathrm{H}), 2.19(\mathrm{~d}, J=6.0 \mathrm{~Hz}, 3 \mathrm{H}) .{ }^{13} \mathrm{C}$ NMR $\left(101 \mathrm{MHz}, \mathrm{CDCl}_{3}\right) \delta 147.5,135.6,129.9,128.8,121.5,13.5 .{ }^{5}$

\section{Typical procedure for the preparation of $\beta$-lactam 2a:}

An oven-dried Schlenk tube equipped with a magnetic stir bar under nitrogen atmosphere was charged with THF $(6 \mathrm{~mL}), N$-methylimidazole $(240 \mu \mathrm{L}, 3 \mathrm{mmol})$, TBAF stock solution $(0.34 \mathrm{mmol}, 680 \mu \mathrm{L})$ and water $(100 \mu \mathrm{L}, 5.6 \mathrm{mmol})$. The solution was deoxygenated by bubbling with nitrogen under stirring at room temperature for $10 \mathrm{~min}$. Copper chloride $(0.074 \mathrm{~g}, 0.76 \mathrm{mmol})$ was added and the mixture was stirred for further $15 \mathrm{~min}$ at the same temperature and then cooled to $-5^{\circ} \mathrm{C}$. The calcium carbide $(0.16$ g, $2.4 \mathrm{mmol})$ and $N$-benzylideneaniline oxide $(0.2 \mathrm{~g}, 1 \mathrm{mmol})$ were sequentially added to the reaction mixture. The reaction progress was followed by GC-MS. Upon completion, the resulting mixture was diluted by EtOAc $(20 \mathrm{~mL})$ and quenched with $\mathrm{H}_{2} \mathrm{O}(2 \mathrm{~mL})$. The mixture was then filtered through a pad of celite. The filterate was washed with brine $(60 \mathrm{~mL})$ and dried over $\mathrm{Na}_{2} \mathrm{SO}_{4}$. The solvent was removed in vacuo and the residual purified by flash column chromatography (silica gel, $5 \% \rightarrow 20 \%$ ethyl acetate $/ n$-hexane) to give $\mathbf{2 a}(0.176 \mathrm{~g}, 79 \%)$. 
$N$-Benzylideneaniline oxide (1a) ${ }^{2}$<smiles>[O-]N(Cc1ccccc1)c1ccccc1</smiles>

${ }^{1} \mathrm{H}$ NMR $\left(400 \mathrm{MHz}, \mathrm{CDCl}_{3}\right) \delta 8.43-8.38(\mathrm{~m}, 2 \mathrm{H}), 7.92(\mathrm{~s}, 1 \mathrm{H}), 7.81-7.75(\mathrm{~m}, 2 \mathrm{H}), 7.51-7.44(\mathrm{~m}$, $6 \mathrm{H}) .{ }^{13} \mathrm{C}$ NMR $\left(100 \mathrm{MHz}, \mathrm{CDCl}_{3}\right) \delta 149.2,134.6,131.0,130.7,130.0,129.2,129.1,128.7,121.8$.

$N$-(4-Carbomethoxyphenyl)- $\alpha$-phenylnitrone (1b) ${ }^{6}$

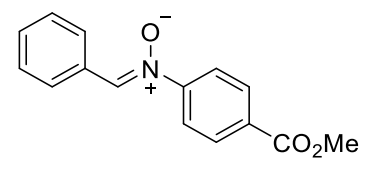

${ }^{1} \mathrm{H}$ NMR $\left(400 \mathrm{MHz}, \mathrm{CDCl}_{3}\right) \delta 8.43-8.40(\mathrm{~m}, 2 \mathrm{H}), 8.16(\mathrm{~d}, J=8.7 \mathrm{~Hz}, 2 \mathrm{H}), 7.98(\mathrm{~s}, 1 \mathrm{H}), 7.86(\mathrm{~d}, J=$ $8.7 \mathrm{~Hz}, 2 \mathrm{H}), 7.50-7.47(\mathrm{~m}, 3 \mathrm{H}), 3.96(\mathrm{~s}, 3 \mathrm{H}) .{ }^{13} \mathrm{C} \mathrm{NMR}\left(100 \mathrm{MHz}, \mathrm{CDCl}_{3}\right) \delta 165.8,152.0,135.1$, $131.5,131.3,130.7,130.4,129.2,128.7,121.8,52.4$.

$\mathrm{N}$-(4-Chlorophenyl)- $\alpha$-phenylnitrone (1c) ${ }^{7}$

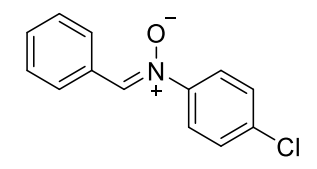

${ }^{1} \mathrm{H}$ NMR $\left(400 \mathrm{MHz}, \mathrm{CDCl}_{3}\right) \delta 8.41-8.36(\mathrm{~m}, 2 \mathrm{H}), 7.90(\mathrm{~s}, 1 \mathrm{H}), 7.75(\mathrm{~d}, J=8.9 \mathrm{~Hz}, 2 \mathrm{H}), 7.51-7.47$ $(\mathrm{m}, 3 \mathrm{H}), 7.45(\mathrm{~d}, J=8.9 \mathrm{~Hz}, 2 \mathrm{H}) .{ }^{13} \mathrm{C}$ NMR $\left(100 \mathrm{MHz}, \mathrm{CDCl}_{3}\right) \delta 147.5,135.8,134.5,131.2,130.5$, $129.3,129.1,128.7,123.1$.

\section{$N$-(4-Methoxyphenyl)- $\alpha$-phenylnitrone (1d) ${ }^{8}$}

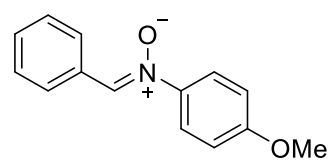

${ }^{1} \mathrm{H}$ NMR $\left(400 \mathrm{MHz}, \mathrm{CDCl}_{3}\right) \delta 8.43-8.34(\mathrm{~m}, 2 \mathrm{H}), 7.87(\mathrm{~s}, 1 \mathrm{H}), 7.72(\mathrm{~d}, J=9.1 \mathrm{~Hz}, 2 \mathrm{H}), 7.50-7.41$ $(\mathrm{m}, 3 \mathrm{H}), 6.96(\mathrm{~d}, J=9.1 \mathrm{~Hz}, 2 \mathrm{H}), 3.86(\mathrm{~s}, 3 \mathrm{H}) .{ }^{13} \mathrm{C} \mathrm{NMR}\left(101 \mathrm{MHz}, \mathrm{CDCl}_{3}\right) \delta 160.6,142.5,133.7$, 130.9, 130.7, 128.9, 128.6, 123.0, 114.1, 55.7.

$\mathrm{N}$-(4-Methoxyphenyl)- $\alpha$-(4-trifluoromethylphenyl)nitrone (1e) ${ }^{9}$

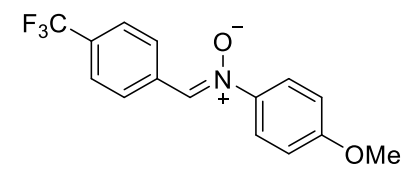

${ }^{1} \mathrm{H} \mathrm{NMR}\left(400 \mathrm{MHz}, \mathrm{CDCl}_{3}\right) \delta 8.48(\mathrm{~d}, J=8.4 \mathrm{~Hz}, 2 \mathrm{H}), 7.94(\mathrm{~s}, 1 \mathrm{H}), 7.74-7.68(\mathrm{~m}, 4 \mathrm{H}), 6.96(\mathrm{~d}, J=$ $9.1 \mathrm{~Hz}, 1 \mathrm{H}), 3.86(\mathrm{~s}, 3 \mathrm{H}) .{ }^{13} \mathrm{C} \mathrm{NMR}\left(101 \mathrm{MHz}, \mathrm{CDCl}_{3}\right) \delta 161.0,142.3,134.0,132.0,131.6\left(\mathrm{q}, J_{\mathrm{C}-\mathrm{F}}=\right.$ $32.5), 128.8,125.5\left(\mathrm{q}, J_{\mathrm{C}-\mathrm{F}}=3.9\right), 123.8\left(\mathrm{q}, J_{\mathrm{C}-\mathrm{F}}=272.8\right), 123.0,114.2,55.7$.

$\mathrm{N}$-(4-Carbomethoxyphenyl)- $\alpha$-(4-methylphenyl)nitrone (1f)

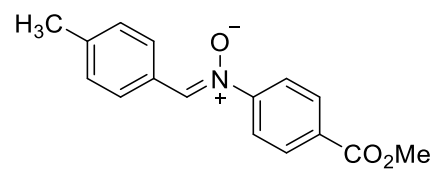

${ }^{1} \mathrm{H}$ NMR $\left(400 \mathrm{MHz}, \mathrm{CDCl}_{3}\right) \delta 8.32(\mathrm{~d}, J=8.3 \mathrm{~Hz}, 2 \mathrm{H}), 8.18-8.14(\mathrm{~m}, 2 \mathrm{H}), 7.94(\mathrm{~s}, 1 \mathrm{H}), 7.89-7.85$ $(\mathrm{m}, 2 \mathrm{H}), 7.31(\mathrm{~d}, J=8.1 \mathrm{~Hz}, 2 \mathrm{H}), 3.96(\mathrm{~s}, 3 \mathrm{H}), 2.43(\mathrm{~s}, 3 \mathrm{H}) .{ }^{13} \mathrm{C} \mathrm{NMR}\left(100 \mathrm{MHz}, \mathrm{CDCl}_{3}\right) \delta 165.9$, $152.0,142.2,135.2,131.4,130.7,129.5,129.4,127.8,121.8,52.5,21.9$. HRMS exact mass calculated for $[\mathrm{M}+\mathrm{Na}]^{+}\left(\mathrm{C}_{16} \mathrm{H}_{15} \mathrm{NO}_{3} \mathrm{Na}^{+}\right): m / z 292.0944$, found $m / z 292.0943$. 


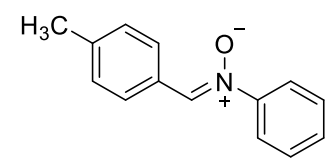

${ }^{1} \mathrm{H}$ NMR $\left(400 \mathrm{MHz}, \mathrm{CDCl}_{3}\right) \delta 8.30(\mathrm{~d}, J=8.3 \mathrm{~Hz}, 2 \mathrm{H}), 7.89(\mathrm{~s}, 1 \mathrm{H}), 7.77(\mathrm{dd}, J=8.1,1.7 \mathrm{~Hz}, 2 \mathrm{H}), 7.52$ $-7.44(\mathrm{~m}, 3 \mathrm{H}), 7.29(\mathrm{~d}, J=8.1 \mathrm{~Hz}, 2 \mathrm{H}), 2.42(\mathrm{~s}, 3 \mathrm{H}) .{ }^{13} \mathrm{C} \mathrm{NMR}\left(101 \mathrm{MHz}, \mathrm{CDCl}_{3}\right) \delta 149.1,141.6$, 134.6, 129.8, 129.4, 129.1, 128.1, 121.8, 21.8.

$N$-Phenyl- $\alpha$-(4-fluorophenyl)nitrone (1h) ${ }^{8}$<smiles>[O-][N+](=Cc1ccc(F)cc1)c1ccccc1</smiles>

${ }^{1} \mathrm{H}$ NMR $\left(400 \mathrm{MHz}, \mathrm{CDCl}_{3}\right) \delta 8.45(\mathrm{dd}, J=8.9,5.6 \mathrm{~Hz}, 2 \mathrm{H}), 7.91(\mathrm{~s}, 1 \mathrm{H}), 7.80-7.73(\mathrm{~m}, 2 \mathrm{H}), 7.53-$ $7.44(\mathrm{~m}, 3 \mathrm{H}), 7.16(\mathrm{t}, J=8.7 \mathrm{~Hz}, 2 \mathrm{H}) .{ }^{13} \mathrm{C} \mathrm{NMR}\left(101 \mathrm{MHz}, \mathrm{CDCl}_{3}\right) \delta 163.7\left(\mathrm{~d}, J_{\mathrm{C}-\mathrm{F}}=253.8 \mathrm{~Hz}\right), 149.0$, $131.3,131.3\left(\mathrm{~d}, J_{\mathrm{C}-\mathrm{F}}=8.3 \mathrm{~Hz}\right), 130.0,129.2,127.2\left(\mathrm{~d}, J_{\mathrm{C}-\mathrm{F}}=3.4 \mathrm{~Hz}\right), 121.7,115.8\left(\mathrm{~d}, J_{\mathrm{C}-\mathrm{F}}=21.7 \mathrm{~Hz}\right)$.

$\mathrm{N}$-Phenyl-a-(4-trifluoromethylphenyl)nitrone (1i) ${ }^{2}$<smiles>[O-]N(Cc1ccc(C(F)(F)F)cc1)c1ccccc1</smiles>

${ }^{1} \mathrm{H}$ NMR $\left(400 \mathrm{MHz}, \mathrm{CDCl}_{3}\right) \delta 8.51(\mathrm{~d}, J=8.3 \mathrm{~Hz}, 2 \mathrm{H}), 8.00(\mathrm{~s}, 1 \mathrm{H}), 7.81-7.76(\mathrm{~m}, 2 \mathrm{H}), 7.73(\mathrm{~d}, J=$ $8.3 \mathrm{~Hz}, 2 \mathrm{H}), 7.55-7.47(\mathrm{~m}, 3 \mathrm{H}) .{ }^{13} \mathrm{C} \mathrm{NMR}\left(101 \mathrm{MHz}, \mathrm{CDCl}_{3}\right) \delta 149.0,133.7,133.1,131.9\left(\mathrm{q}, J_{\mathrm{C}-\mathrm{F}}=\right.$ $32.4 \mathrm{~Hz}), 130.4,129.3,128.9,125.6\left(\mathrm{q}, J_{\mathrm{C}-\mathrm{F}}=3.8 \mathrm{~Hz}\right), 123.8\left(\mathrm{q}, J_{\mathrm{C}-\mathrm{F}}=272.5 \mathrm{~Hz}\right), 121.8$

$\mathrm{N}$-(2-Methoxyphenyl)- $\alpha$-phenylnitrone $(\mathbf{1 j})^{\mathbf{1 0}}$<smiles>COc1ccccc1/C=N/c1ccccc1</smiles>

${ }^{1} \mathrm{H}$ NMR $\left(400 \mathrm{MHz}, \mathrm{CDCl}_{3}\right) \delta 8.40-8.32(\mathrm{~m}, 2 \mathrm{H}), 7.63(\mathrm{~s}, 1 \mathrm{H}), 7.60(\mathrm{dd}, J=8.2,1.7 \mathrm{~Hz}, 1 \mathrm{H}), 7.46-$ $7.41(\mathrm{~m}, 3 \mathrm{H}), 7.39-7.33(\mathrm{~m}, 1 \mathrm{H}), 7.03-6.99(\mathrm{~m}, 2 \mathrm{H}), 3.85(\mathrm{~s}, 3 \mathrm{H}) .{ }^{13} \mathrm{C} \mathrm{NMR}\left(101 \mathrm{MHz}, \mathrm{CDCl}_{3}\right) \delta$ $151.4,139.1,138.6,130.8,130.6,130.6,129.1,128.6,125.3,120.9,112.5,56.2$.

$\mathrm{N}$-(2-Chlorophenyl)- $\alpha$-phenylnitrone (1k) ${ }^{11}$<smiles>O=[N+]([O-])c1ccccc1Cl</smiles>

${ }^{1} \mathrm{H}$ NMR $\left(400 \mathrm{MHz}, \mathrm{CDCl}_{3}\right) \delta 8.39-8.34(\mathrm{~m}, 2 \mathrm{H}), 7.64-7.60(\mathrm{~m}, 1 \mathrm{H}), 7.58(\mathrm{~s}, 1 \mathrm{H}), 7.54-7.46(\mathrm{~m}$, $4 \mathrm{H}), 7.41-7.37(\mathrm{~m}, 2 \mathrm{H}) .{ }^{13} \mathrm{C} \mathrm{NMR}\left(101 \mathrm{MHz}, \mathrm{CDCl}_{3}\right) \delta 146.7,139.0,131.3,130.7,130.5,130.1$, $129.1,128.7,127.7,127.4,125.7$.

$\mathrm{N}$-(4-Fluorophenyl)-a-(4-benzyloxyphenyl)nitrone (1m) ${ }^{1}$<smiles>O=C(OCc1ccccc1)Oc1ccc(C=Nc2ccc(F)cc2)cc1</smiles>

${ }^{1} \mathrm{H}$ NMR (400 MHz, DMSO- $\left.d_{6}\right) \delta 8.49-8.45(\mathrm{~m}, 2 \mathrm{H}), 8.42(\mathrm{~s}, 1 \mathrm{H}), 8.00-7.94(\mathrm{~m}, 2 \mathrm{H}), 7.51-7.45$ $(\mathrm{m}, 2 \mathrm{H}), 7.45-7.31(\mathrm{~m}, 5 \mathrm{H}), 7.18-7.12(\mathrm{~m}, 2 \mathrm{H}), 5.20(\mathrm{~s}, 2 \mathrm{H}) .{ }^{13} \mathrm{C}$ NMR $\left(101 \mathrm{MHz}, \mathrm{DMSO}-d_{6}\right) \delta$ $162.1\left(\mathrm{~d}, J_{\mathrm{C}-\mathrm{F}}=247.6 \mathrm{~Hz}\right), 159.9,144.8\left(\mathrm{~d}, J_{\mathrm{C}-\mathrm{F}}=2.5 \mathrm{~Hz}\right), 136.6,133.0,130.9,128.5,128.0,127.8$, $124.1,123.6\left(\mathrm{~d}, J_{\mathrm{C}-\mathrm{F}}=8.9 \mathrm{~Hz}\right), 115.7\left(\mathrm{~d}, J_{\mathrm{C}-\mathrm{F}}=23.2 \mathrm{~Hz}\right), 114.7,69.4$. 


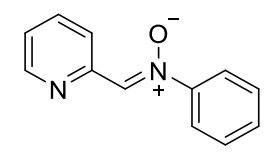

${ }^{1} \mathrm{H}$ NMR $\left(400 \mathrm{MHz}, \mathrm{CDCl}_{3}\right) \delta 9.33(\mathrm{~d}, J=8.1 \mathrm{~Hz}, 1 \mathrm{H}), 8.69-8.66(\mathrm{~m}, 1 \mathrm{H}), 8.27(\mathrm{~s}, 1 \mathrm{H}), 7.87-7.79$ $(\mathrm{m}, 3 \mathrm{H}), 7.50-7.46(\mathrm{~m}, 3 \mathrm{H}), 7.34-7.29(\mathrm{~m}, 1 \mathrm{H}) .{ }^{13} \mathrm{C} \mathrm{NMR}\left(101 \mathrm{MHz}, \mathrm{CDCl}_{3}\right) \delta 149.9,149.8,148.8$, $137.0,135.5,130.4,129.3,124.6,124.0,121.7$.

$\mathrm{N}$-Phenyl- $\alpha-3$-pyridylnitrone (10) ${ }^{13}$

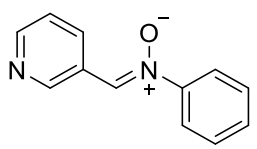

${ }^{1} \mathrm{H}$ NMR $\left(400 \mathrm{MHz}, \mathrm{CDCl}_{3}\right) \delta 9.20-9.15(\mathrm{~m}, 1 \mathrm{H}), 9.08(\mathrm{~d}, J=2.2 \mathrm{~Hz}, 1 \mathrm{H}), 8.63(\mathrm{dd}, J=4.9,1.7 \mathrm{~Hz}$, $1 \mathrm{H}), 7.97(\mathrm{~s}, 1 \mathrm{H}), 7.79-7.73(\mathrm{~m}, 2 \mathrm{H}), 7.53-7.44(\mathrm{~m}, 3 \mathrm{H}), 7.40(\mathrm{dd}, J=8.1,4.8 \mathrm{~Hz}, 1 \mathrm{H}) .{ }^{13} \mathrm{C}$ NMR $\left(101 \mathrm{MHz}, \mathrm{CDCl}_{3}\right) \delta 150.9,150.4,148.8,134.8,131.5,130.4,129.31,127.3,123.7,121.7$.

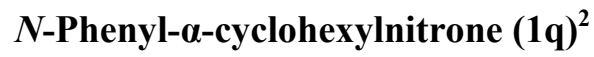

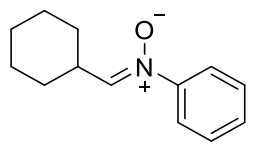

${ }^{1} \mathrm{H}$ NMR $\left(400 \mathrm{MHz}, \mathrm{CDCl}_{3}\right) \delta 7.66-7.63(\mathrm{~m}, 2 \mathrm{H}), 7.44-7.38(\mathrm{~m}, 3 \mathrm{H}), 7.03(\mathrm{~d}, J=7.5 \mathrm{~Hz}, 1 \mathrm{H}), 3.24$ $-3.15(\mathrm{~m}, 1 \mathrm{H}), 2.06-1.94(\mathrm{~m}, 2 \mathrm{H}), 1.79-1.67(\mathrm{~m}, 3 \mathrm{H}), 1.51-1.38(\mathrm{~m}, 2 \mathrm{H}), 1.34-1.22(\mathrm{~m}, 3 \mathrm{H}) .{ }^{13} \mathrm{C}$ $\operatorname{NMR}\left(101 \mathrm{MHz}, \mathrm{CDCl}_{3}\right) \delta 147.9,143.5,129.8,129.0,121.7,35.7,28.9,26.0,25.3$.

$\mathrm{N}$-Cyclohexyl- $\alpha$-phenylnitrone $(1 \mathrm{r})^{14}$

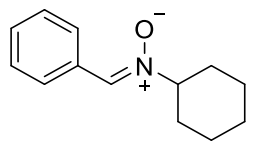

${ }^{1} \mathrm{H}$ NMR $\left(400 \mathrm{MHz}, \mathrm{CDCl}_{3}\right) \delta 8.27-8.23(\mathrm{~m}, 2 \mathrm{H}), 7.45-7.35(\mathrm{~m}, 4 \mathrm{H}), 3.85(\mathrm{tt}, J=11.5,3.9 \mathrm{~Hz}, 1 \mathrm{H})$, $2.12-1.86(\mathrm{~m}, 6 \mathrm{H}), 1.74-1.67(\mathrm{~m}, 1 \mathrm{H}), 1.43-1.19(\mathrm{~m}, 3 \mathrm{H}) .{ }^{13} \mathrm{C} \mathrm{NMR}\left(101 \mathrm{MHz}, \mathrm{CDCl}_{3}\right) \delta 132.2$, $130.8,130.1,128.6,128.5,75.7,31.2,25.1,25.1$.

$\mathrm{N}$-Methyl-a-phenylnitrone (1s) ${ }^{4}$

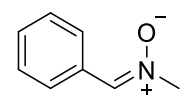

${ }^{1} \mathrm{H}$ NMR $\left(400 \mathrm{MHz}, \mathrm{CDCl}_{3}\right) \delta 8.22-8.18(\mathrm{~m}, 2 \mathrm{H}), 7.44-7.38(\mathrm{~m}, 3 \mathrm{H}), 7.36(\mathrm{~s}, 1 \mathrm{H}), 3.86(\mathrm{~s}, 3 \mathrm{H}) .{ }^{13} \mathrm{C}$ NMR $\left(101 \mathrm{MHz}, \mathrm{CDCl}_{3}\right) \delta 135.2,130.5,130.4,128.5,128.4,54.4$. 


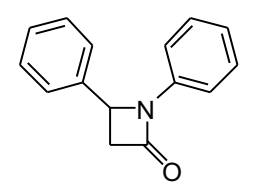

White solid $(0.176 \mathrm{~g}, 79 \%)$, purified by silica gel chromatography $(5 \% \rightarrow 20 \%$ ethyl acetate $/ n$-hexane). ${ }^{1} \mathrm{H}$ NMR $\left(400 \mathrm{MHz}, \mathrm{CDCl}_{3}\right) \delta 7.40-7.25(\mathrm{~m}, 7 \mathrm{H}), 7.25-7.18(\mathrm{~m}, 2 \mathrm{H}), 7.01(\mathrm{t}, J=7.3 \mathrm{~Hz}, 1 \mathrm{H}), 4.98$ $(\mathrm{dd}, J=5.7,2.6 \mathrm{~Hz}, 1 \mathrm{H}), 3.53(\mathrm{dd}, J=15.1,5.7 \mathrm{~Hz}, 1 \mathrm{H}), 2.92(\mathrm{dd}, J=15.1,2.7 \mathrm{~Hz}, 1 \mathrm{H}) .{ }^{13} \mathrm{C}$ NMR $\left(101 \mathrm{MHz}, \mathrm{CDCl}_{3}\right) \delta 164.6,138.3,137.8,129.2,129.1,128.6,125.9,123.9,116.8,54.0,47.1$. HRMS exact mass calculated for $[\mathrm{M}+\mathrm{Na}]^{+}\left(\mathrm{C}_{15} \mathrm{H}_{13} \mathrm{NONa}^{+}\right): m / z 246.0889$, found $m / z 246.0891$.

\section{1-(4-Carbomethoxyphenyl)-4-phenyl-2-azetidinone (2b)}

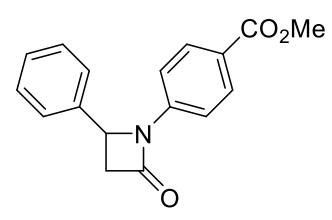

Light yellow solid $(0.24 \mathrm{~g}, 85 \%)$, purified by silica gel chromatography $(5 \% \rightarrow 20 \%$ ethyl acetate $/ n$ hexane). ${ }^{1} \mathrm{H}$ NMR $\left(400 \mathrm{MHz}, \mathrm{CDCl}_{3}\right) \delta 7.95-7.89(\mathrm{~m}, 2 \mathrm{H}), 7.43-7.29(\mathrm{~m}, 7 \mathrm{H}), 5.06(\mathrm{dd}, J=5.8,2.7$ $\mathrm{Hz}, 1 \mathrm{H}), 3.86(\mathrm{~s}, 3 \mathrm{H}), 3.61(\mathrm{dd}, J=15.4,5.8 \mathrm{~Hz}, 1 \mathrm{H}), 3.00(\mathrm{dd}, J=15.4,2.8 \mathrm{~Hz}, 1 \mathrm{H}) .{ }^{13} \mathrm{C} \mathrm{NMR}(101$ $\left.\mathrm{MHz}, \mathrm{CDCl}_{3}\right) \delta 166.5,164.9,141.4,137.7,130.9,129.3,128.8,125.9,125.3,116.3,54.4,52.0,47.4$. HRMS exact mass calculated for $[\mathrm{M}+\mathrm{Na}]^{+}\left(\mathrm{C}_{17} \mathrm{H}_{15} \mathrm{NO}_{3} \mathrm{Na}^{+}\right): \mathrm{m} / z$ 304.0944, found $\mathrm{m} / z$ 304.0942.

\section{1-(4-Chlorophenyl)-4-phenyl-2-azetidinone (2c) ${ }^{16}$}

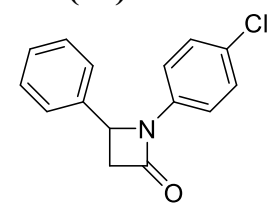

Light yellow solid $(0.2 \mathrm{~g}, 78 \%)$, purified by silica gel chromatography $(5 \% \rightarrow 20 \%$ ethyl acetate $/ n$ hexane). ${ }^{1} \mathrm{H}$ NMR (400 MHz, $\left.\mathrm{CDCl}_{3}\right) \delta 7.42-7.31(\mathrm{~m}, 5 \mathrm{H}), 7.21(\mathrm{q}, J=9.0 \mathrm{~Hz}, 4 \mathrm{H}), 4.99$ (dd, $J=5.7$, $2.7 \mathrm{~Hz}, 2 \mathrm{H}), 3.57(\mathrm{dd}, J=15.2,5.7 \mathrm{~Hz}, 2 \mathrm{H}), 2.96(\mathrm{dd}, J=15.2,2.7 \mathrm{~Hz}, 2 \mathrm{H}) .{ }^{13} \mathrm{C}$ NMR $(101 \mathrm{MHz}$, $\left.\mathrm{CDCl}_{3}\right) \delta 164.5,137.8,136.3,129.3,129.2,128.9,128.7,125.9,118.0,54.2,47.3$. HRMS exact mass calculated for $[\mathrm{M}+\mathrm{Na}]^{+}\left(\mathrm{C}_{15} \mathrm{H}_{12} \mathrm{ClNONa}^{+}\right): \mathrm{m} / z$ 280.0500, found $\mathrm{m} / \mathrm{z} 280.0501$.

\section{1-(4-Methoxyphenyl)-4-phenyl-2-azetidinone (2d) $)^{17}$}

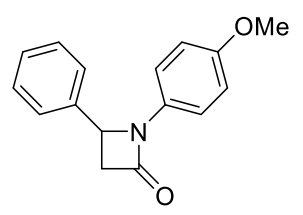

White solid $(0.11 \mathrm{~g}, 44 \%)$, purified by silica gel chromatography $(5 \% \rightarrow 20 \%$ ethyl acetate $/ n$-hexane). ${ }^{1} \mathrm{H}$ NMR $\left(400 \mathrm{MHz}, \mathrm{CDCl}_{3}\right) \delta 7.42-7.26(\mathrm{~m}, 5 \mathrm{H}), 7.23(\mathrm{~d}, J=9.0 \mathrm{~Hz}, 2 \mathrm{H}), 6.77(\mathrm{~d}, J=9.0 \mathrm{~Hz}, 2 \mathrm{H})$, $4.96(\mathrm{dd}, J=5.6,2.6 \mathrm{~Hz}, 1 \mathrm{H}), 3.73(\mathrm{~s}, 3 \mathrm{H}), 3.53(\mathrm{dd}, J=15.0,5.6 \mathrm{~Hz}, 1 \mathrm{H}), 2.92(\mathrm{dd}, J=15.0,2.6 \mathrm{~Hz}$, $1 \mathrm{H}) .{ }^{13} \mathrm{C}$ NMR $\left(101 \mathrm{MHz}, \mathrm{CDCl}_{3}\right) \delta 164.0,156.0,138.4,131.5,129.2,128.5,126.0,118.1,114.3,55.4$, 54.1, 47.0. HRMS exact mass calculated for $[\mathrm{M}+\mathrm{Na}]^{+}\left(\mathrm{C}_{16} \mathrm{H}_{15} \mathrm{NO}_{2} \mathrm{Na}^{+}\right): m / z 276.0995$, found $\mathrm{m} / \mathrm{z}$ 276.0998 . 


\section{1-(4-Methoxyphenyl)-4-[4-(trifluoromethyl)phenyl]-2-azetidinone (2e) ${ }^{18}$}

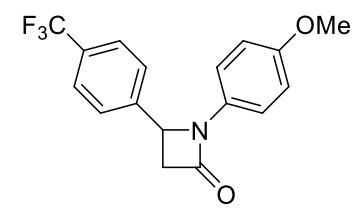

Yellow solid ( $0.18 \mathrm{~g}, 56 \%)$, purified by silica gel chromatography $(5 \% \rightarrow 20 \%$ ethyl acetate $/ n$-hexane). ${ }^{1} \mathrm{H}$ NMR $\left(400 \mathrm{MHz}, \mathrm{CDCl}_{3}\right) \delta 7.64(\mathrm{~d}, J=8.1 \mathrm{~Hz}, 2 \mathrm{H}), 7.49(\mathrm{~d}, J=8.1 \mathrm{~Hz}, 2 \mathrm{H}), 7.20(\mathrm{~d}, J=9.0 \mathrm{~Hz}$, $2 \mathrm{H}), 6.80(\mathrm{~d}, J=9.1 \mathrm{~Hz}, 2 \mathrm{H}), 5.04(\mathrm{dd}, J=5.6,2.5 \mathrm{~Hz}, 1 \mathrm{H}), 3.74(\mathrm{~s}, 3 \mathrm{H}), 3.59(\mathrm{dd}, J=15.0,5.6 \mathrm{~Hz}$, $1 \mathrm{H}), 2.91(\mathrm{dd}, J=15.0,2.6 \mathrm{~Hz}, 1 \mathrm{H}) .{ }^{13} \mathrm{C}$ NMR $\left(101 \mathrm{MHz}, \mathrm{CDCl}_{3}\right) \delta 163.4,156.2,142.5,131.1,130.8$ $\left(\mathrm{q}, J_{\mathrm{C}-\mathrm{F}}=32.8 \mathrm{~Hz}\right), 126.3,126.2\left(\mathrm{q}, J_{\mathrm{C}-\mathrm{F}}=3.8 \mathrm{~Hz}\right), 125.2,118.1,114.4,55.5,53.5,47.0$. HRMS exact mass calculated for $[\mathrm{M}+\mathrm{Na}]^{+}\left(\mathrm{C}_{17} \mathrm{H}_{14} \mathrm{~F}_{3} \mathrm{NO}_{2} \mathrm{Na}^{+}\right): m / z 344.0869$, found $m / z$ 344.0866.

\section{1-(4-Carbomethoxyphenyl)-4-[4-methylphenyl]-2-azetidinone (2f)}

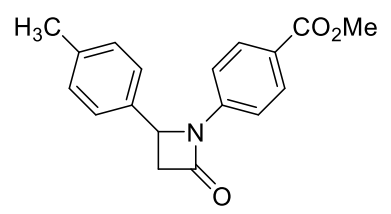

White solid $(0.27 \mathrm{~g}, 90 \%)$, purified by silica gel chromatography $(5 \% \rightarrow 20 \%$ ethyl acetate $/ n$-hexane). ${ }^{1} \mathrm{H}$ NMR $\left(400 \mathrm{MHz}, \mathrm{CDCl}_{3}\right) \delta 7.92(\mathrm{~d}, J=8.8 \mathrm{~Hz}, 2 \mathrm{H}), 7.32(\mathrm{~d}, J=8.8 \mathrm{~Hz}, 2 \mathrm{H}), 7.29-7.23(\mathrm{~m}, 2 \mathrm{H})$, $7.21-7.16(\mathrm{~m}, 2 \mathrm{H}), 5.02(\mathrm{dd}, J=5.8,2.8 \mathrm{~Hz}, 1 \mathrm{H}), 3.86(\mathrm{~s}, 3 \mathrm{H}), 3.58(\mathrm{dd}, J=15.4,5.8 \mathrm{~Hz}, 1 \mathrm{H}), 2.97$ $(\mathrm{dd}, J=15.4,2.8 \mathrm{~Hz}, 1 \mathrm{H}), 2.35(\mathrm{~s}, 3 \mathrm{H}) .{ }^{13} \mathrm{C} \mathrm{NMR}\left(101 \mathrm{MHz}, \mathrm{CDCl}_{3}\right) \delta 166.5,165.0,141.4,138.7$, 134.6, 130.9, 130.0, 125.8, 125.2, 116.3, 54.2, 52.0, 47.4, 21.2. HRMS exact mass calculated for $[\mathrm{M}+\mathrm{Na}]^{+}\left(\mathrm{C}_{18} \mathrm{H}_{17} \mathrm{NO}_{3} \mathrm{Na}^{+}\right): m / z$ 318.1100, found $m / z$ 318.1102.

\section{1-Phenyl-4-[4-methylphenyl]-2-azetidinone (2g) $)^{19}$}

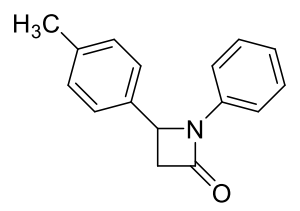

White solid $(0.133 \mathrm{~g}, 56 \%)$, purified by silica gel chromatography ( $5 \% \rightarrow 20 \%$ ethyl acetate $/ n$-hexane). ${ }^{1} \mathrm{H}$ NMR $\left(400 \mathrm{MHz}, \mathrm{CDCl}_{3}\right) \delta 7.31-7.19(\mathrm{~m}, 6 \mathrm{H}), 7.19-7.15(\mathrm{~m}, 2 \mathrm{H}), 7.05-6.99(\mathrm{~m}, 1 \mathrm{H}), 4.97$ (dd, $J=5.7,2.6 \mathrm{~Hz}, 1 \mathrm{H}), 3.52(\mathrm{dd}, J=15.1,5.7 \mathrm{~Hz}, 1 \mathrm{H}), 2.92(\mathrm{dd}, J=15.1,2.6 \mathrm{~Hz}, 1 \mathrm{H}), 2.34(\mathrm{~s}, 3 \mathrm{H}) .{ }^{13} \mathrm{C}$ NMR (101 MHz, $\left.\mathrm{CDCl}_{3}\right) \delta 164.7,138.4,137.9,135.2,129.9,129.0,125.9,123.8,116.8,53.9,47.1$, 21.2. HRMS exact mass calculated for $[\mathrm{M}+\mathrm{Na}]^{+}\left(\mathrm{C}_{16} \mathrm{H}_{15} \mathrm{NONa}^{+}\right): \mathrm{m} / z 260.1046$, found $\mathrm{m} / z 260.1046$.

\section{1-Phenyl-4-[4-fluorophenyl]-2-azetidinone (2h) $)^{20}$}

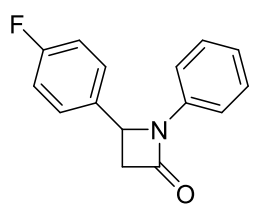

White solid ( $0.16 \mathrm{~g}, 66 \%)$, purified by silica gel chromatography $(5 \% \rightarrow 20 \%$ ethyl acetate $/ n$-hexane). ${ }^{1} \mathrm{H}$ NMR $\left(400 \mathrm{MHz}, \mathrm{CDCl}_{3}\right) \delta 7.38-7.33(\mathrm{~m}, 2 \mathrm{H}), 7.30-7.21(\mathrm{~m}, 4 \mathrm{H}), 7.11-7.00(\mathrm{~m}, 3 \mathrm{H}), 5.00(\mathrm{dd}$, $J=5.7,2.6 \mathrm{~Hz}, 1 \mathrm{H}), 3.56(\mathrm{dd}, J=15.2,5.7 \mathrm{~Hz}, 1 \mathrm{H}), 2.92(\mathrm{dd}, J=15.2,2.7 \mathrm{~Hz}, 1 \mathrm{H}) .{ }^{13} \mathrm{C}$ NMR $(101$ $\left.\mathrm{MHz}, \mathrm{CDCl}_{3}\right) \delta 164.4,162.72\left(\mathrm{~d}, J_{\mathrm{C}-\mathrm{F}}=247.5 \mathrm{~Hz}\right), 137.6,134.0\left(\mathrm{~d}, J_{\mathrm{C}-\mathrm{F}}=3.2 \mathrm{~Hz}\right), 129.1,127.65\left(\mathrm{~d}, J_{\mathrm{C}-}\right.$ $\mathrm{F}=8.3 \mathrm{~Hz}), 124.0,116.8,116.2\left(\mathrm{~d}, J_{\mathrm{C}-\mathrm{F}}=21.8 \mathrm{~Hz}\right), 53.4,47.1$. HRMS exact mass calculated for $[\mathrm{M}+\mathrm{Na}]^{+}\left(\mathrm{C}_{15} \mathrm{H}_{12} \mathrm{FNONa}^{+}\right): \mathrm{m} / z$ 264.0795, found $\mathrm{m} / \mathrm{z} 264.0792$. 


\section{1-Phenyl-4-[4-(trifluoromethyl)phenyl]-2-azetidinone (2i)}

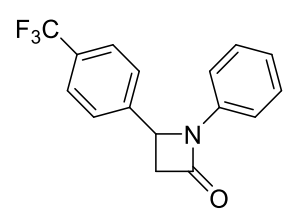

Light yellow solid $(0.175 \mathrm{~g}, 60 \%)$, purified by silica gel chromatography $(5 \% \rightarrow 20 \%$ ethyl acetate $/ n$ hexane). ${ }^{1} \mathrm{H}$ NMR $\left(400 \mathrm{MHz}, \mathrm{CDCl}_{3}\right) \delta 7.64(\mathrm{~d}, J=8.0 \mathrm{~Hz}, 2 \mathrm{H}), 7.50(\mathrm{~d}, J=8.1 \mathrm{~Hz}, 2 \mathrm{H}), 7.27-7.24$ $(\mathrm{m}, 4 \mathrm{H}), 7.11-7.01(\mathrm{~m}, 1 \mathrm{H}), 5.08(\mathrm{dd}, J=5.8,2.6 \mathrm{~Hz}, 1 \mathrm{H}), 3.61(\mathrm{dd}, J=15.2,5.8 \mathrm{~Hz}, 1 \mathrm{H}), 2.93(\mathrm{dd}$, $J=15.2,2.7 \mathrm{~Hz}, 1 \mathrm{H}) .{ }^{13} \mathrm{C} \mathrm{NMR}\left(101 \mathrm{MHz}, \mathrm{CDCl}_{3}\right) \delta 164.0,142.4,137.5,130.8\left(\mathrm{q}, J_{\mathrm{C}-\mathrm{F}}=32.6 \mathrm{~Hz}\right)$, $129.2,126.3\left(\mathrm{q}, J_{\mathrm{C}-\mathrm{F}}=3.8 \mathrm{~Hz}\right), 126.3,124.2,123.9\left(\mathrm{q}, J_{\mathrm{C}-\mathrm{F}}=273.1\right), 116.7,53.4,47.0$. HRMS exact mass calculated for $[\mathrm{M}+\mathrm{Na}]^{+}\left(\mathrm{C}_{16} \mathrm{H}_{12} \mathrm{~F}_{3} \mathrm{NONa}^{+}\right): m / z 314.0763$, found $m / z$ 314.0764.

\section{1-(2-Methoxyphenyl)-4-phenyl-2-azetidinone (2j)}

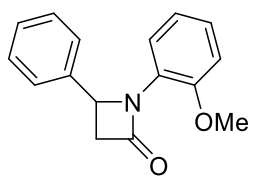

Yellow oil ( $0.117 \mathrm{~g}, 46 \%)$, purified by silica gel chromatography ( $5 \% \rightarrow 20 \%$ ethyl acetate $/ n$-hexane). ${ }^{1} \mathrm{H}$ NMR $\left(400 \mathrm{MHz}, \mathrm{CDCl}_{3}\right) \delta 7.85(\mathrm{dd}, J=7.9,1.7 \mathrm{~Hz}, 1 \mathrm{H}), 7.32-7.19(\mathrm{~m}, 5 \mathrm{H}), 7.06-7.01(\mathrm{~m}, 1 \mathrm{H})$, $6.90(\mathrm{td}, J=7.7,1.3 \mathrm{~Hz}, 1 \mathrm{H}), 6.76(\mathrm{dd}, J=8.2,1.3 \mathrm{~Hz}, 1 \mathrm{H}), 5.39$ (dd, $J=5.6,2.6 \mathrm{~Hz}, 1 \mathrm{H}), 3.59(\mathrm{~s}, 3 \mathrm{H})$, $3.53(\mathrm{dd}, J=15.0,5.6 \mathrm{~Hz}, 1 \mathrm{H}), 2.94(\mathrm{dd}, J=15.0,2.6 \mathrm{~Hz}, 1 \mathrm{H}) .{ }^{13} \mathrm{C} \mathrm{NMR}\left(101 \mathrm{MHz}, \mathrm{CDCl}_{3}\right) \delta 165.9$, $150.8,140.0,128.7,127.9,125.9,125.8,125.7,123.4,121.0,112.0,57.6,55.4,47.6$. HRMS exact mass calculated for $[\mathrm{M}+\mathrm{Na}]^{+}\left(\mathrm{C}_{16} \mathrm{H}_{15} \mathrm{NO}_{2} \mathrm{Na}^{+}\right): \mathrm{m} / z 276.0995$, found $\mathrm{m} / z 276.0993$.

\section{1-(2-Chlorophenyl)-4-phenyl-2-azetidinone (2k)}

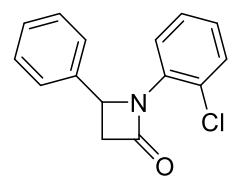

Yellow solid $(0.165 \mathrm{~g}, 64 \%)$, purified by silica gel chromatography $(5 \% \rightarrow 20 \%$ ethyl acetate $/ n$-hexane). ${ }^{1} \mathrm{H}$ NMR $\left(400 \mathrm{MHz}, \mathrm{CDCl}_{3}\right) \delta 7.68(\mathrm{dd}, J=8.0,1.6 \mathrm{~Hz}, 1 \mathrm{H}), 7.36-7.21(\mathrm{~m}, 6 \mathrm{H}), 7.18(\mathrm{td}, J=7.7,1.5$ $\mathrm{Hz}, 1 \mathrm{H}), 7.03(\mathrm{td}, J=7.7,1.6 \mathrm{~Hz}, 1 \mathrm{H}), 5.53(\mathrm{dd}, J=5.6,2.6 \mathrm{~Hz}, 1 \mathrm{H}), 3.57(\mathrm{dd}, J=15.2,5.6 \mathrm{~Hz}, 1 \mathrm{H})$, $3.01(\mathrm{dd}, J=15.2,2.6 \mathrm{~Hz}, 1 \mathrm{H}) .{ }^{13} \mathrm{C} \mathrm{NMR}\left(101 \mathrm{MHz}, \mathrm{CDCl}_{3}\right) \delta 165.8,138.6,133.4,130.7,129.0,128.5$, $127.5,127.1,126.5,126.3,125.6,57.4,47.2$. HRMS exact mass calculated for $[\mathrm{M}+\mathrm{Na}]^{+}$ $\left(\mathrm{C}_{15} \mathrm{H}_{12} \mathrm{ClNONa}^{+}\right): \mathrm{m} / z 280.0499$, found $\mathrm{m} / z 280.0500$.

1-(4-Cyano-3-trifluoromethylphenyl)-4-phenyl-2-azetidinone (2l) ${ }^{21}$<smiles>N#Cc1ccc(N2C(=O)CC2c2ccccc2)cc1C(F)(F)F</smiles>

Light yellow solid $(0.272 \mathrm{~g}, 86 \%)$, purified by silica gel chromatography $(5 \% \rightarrow 25 \%$ ethyl acetate $/ n$ hexane). ${ }^{1} \mathrm{H}$ NMR $\left(400 \mathrm{MHz}, \mathrm{CDCl}_{3}\right) \delta 7.81(\mathrm{~d}, J=2.1 \mathrm{~Hz}, 1 \mathrm{H}), 7.66(\mathrm{~d}, J=8.4 \mathrm{~Hz}, 1 \mathrm{H}), 7.46-7.33$ (m, 6H), $5.11(\mathrm{dd}, J=6.0,2.9 \mathrm{~Hz}, 1 \mathrm{H}), 3.69(\mathrm{dd}, J=15.8,5.9 \mathrm{~Hz}, 1 \mathrm{H}), 3.10(\mathrm{dd}, J=15.8,2.9 \mathrm{~Hz}, 1 \mathrm{H})$. ${ }^{13} \mathrm{C}$ NMR $\left(101 \mathrm{MHz}, \mathrm{CDCl}_{3}\right) \delta 165.1,141.1,136.4,135.9,134.2\left(\mathrm{q}, J_{\mathrm{C}-\mathrm{F}}=32.8 \mathrm{~Hz}\right), 129.6,129.4,125.9$, $121.9\left(\mathrm{q}, J_{\mathrm{C}-\mathrm{F}}=274.2 \mathrm{~Hz}\right), 118.9,115.5,115.0\left(\mathrm{q}, J_{\mathrm{C}-\mathrm{F}}=5.0 \mathrm{~Hz}\right), 103.91\left(\mathrm{q}, J_{\mathrm{C}-\mathrm{F}}=2.2 \mathrm{~Hz}\right), 54.9,47.8$. HRMS exact mass calculated for [M+Na] $]^{+}\left(\mathrm{C}_{17} \mathrm{H}_{11} \mathrm{~F}_{3} \mathrm{~N}_{2} \mathrm{ONa}^{+}\right): \mathrm{m} / z$ 339.0716, found $m / z 339.0714$. 


\section{1-(4-Fluorophenyl)-4-[4-(benzyloxy)phenyl]-2-azetidinone (2m) ${ }^{20}$}

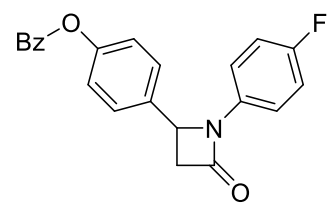

White solid $(0.2 \mathrm{~g}, 58 \%)$, purified by silica gel chromatography $\left(5 \% \rightarrow 30 \%\right.$ ethyl acetate $/ n$-hexane). ${ }^{1} \mathrm{H}$ $\operatorname{NMR}\left(400 \mathrm{MHz}, \mathrm{CDCl}_{3}\right) \delta 7.43-7.22(\mathrm{~m}, 9 \mathrm{H}), 6.99-6.88(\mathrm{~m}, 4 \mathrm{H}), 5.03(\mathrm{~s}, 2 \mathrm{H}), 4.91(\mathrm{dd}, J=5.6,2.6$ $\mathrm{Hz}, 1 \mathrm{H}), 3.50(\mathrm{dd}, J=15.2,5.6 \mathrm{~Hz}, 1 \mathrm{H}), 2.91(\mathrm{dd}, J=15.2,2.6 \mathrm{~Hz}, 1 \mathrm{H}) .{ }^{13} \mathrm{C} \mathrm{NMR}\left(101 \mathrm{MHz}, \mathrm{CDCl}_{3}\right)$ $\delta 164.5,160.2,159.1,157.8,136.7,134.2,134.1,130.1,128.7,128.1,127.5,127.3,118.3,118.2,116.0$, 115.7, 115.6, 70.1, 53.9, 47.3. HRMS exact mass calculated for $[\mathrm{M}+\mathrm{Na}]^{+}\left(\mathrm{C}_{22} \mathrm{H}_{18} \mathrm{FNO}_{2} \mathrm{Na}^{+}\right): \mathrm{m} / z$ 370.1214 , found $\mathrm{m} / \mathrm{z} 370.1213$.

\section{1-Phenyl-4-[2-pyridyl]-2-azetidinone (2n)}

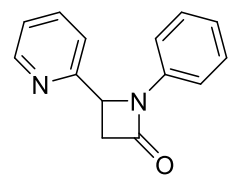

Light yellow solid $(0.125 \mathrm{~g}, 56 \%)$, purified by silica gel chromatography $(20 \% \rightarrow 80 \%$ ethyl acetate $/ n$ hexane). ${ }^{1} \mathrm{H}$ NMR $\left(400 \mathrm{MHz}, \mathrm{CDCl}_{3}\right) \delta 8.66-8.59(\mathrm{~m}, 1 \mathrm{H}), 7.65-8.71(\mathrm{~m}, 1 \mathrm{H}), 7.35(\mathrm{~d}, J=7.9 \mathrm{~Hz}$, $1 \mathrm{H}), 7.32-7.21(\mathrm{~m}, 5 \mathrm{H}), 7.06-7.01(\mathrm{~m}, 1 \mathrm{H}), 5.15(\mathrm{dd}, J=5.8,2.6 \mathrm{~Hz}, 1 \mathrm{H}), 3.58(\mathrm{dd}, J=15.1,5.8 \mathrm{~Hz}$, $1 \mathrm{H}), 3.05(\mathrm{dd}, J=15.1,2.6 \mathrm{~Hz}, 1 \mathrm{H}) .{ }^{13} \mathrm{C} \mathrm{NMR}\left(101 \mathrm{MHz}, \mathrm{CDCl}_{3}\right) \delta 164.4,157.9,150.0,137.7,137.3$, $129.1,123.9,123.4,120.3,116.7,55.0,45.5$. HRMS exact mass calculated for $[\mathrm{M}+\mathrm{Na}]^{+}$ $\left(\mathrm{C}_{14} \mathrm{H}_{12} \mathrm{~N}_{2} \mathrm{ONa}^{+}\right): m / z 247.0842$, found $m / z 247.0845$.

\section{1-Phenyl-4-[3-pyridyl]-2-azetidinone (2o) ${ }^{22}$}<smiles>O=C1CC(c2cccnc2)N(c2ccccc2)C1</smiles>

Light yellow solid $(0.134 \mathrm{~g}, 60 \%)$, purified by silica gel chromatography $(20 \% \rightarrow 80 \%$ ethyl acetate $/ n$ hexane). ${ }^{1} \mathrm{H}$ NMR $\left(400 \mathrm{MHz}, \mathrm{CDCl}_{3}\right) \delta 8.69(\mathrm{~d}, J=2.3 \mathrm{~Hz}, 1 \mathrm{H}), 8.61(\mathrm{dd}, J=4.8,1.7 \mathrm{~Hz}, 1 \mathrm{H}), 7.69$ $(\mathrm{dt}, J=7.9,2.0 \mathrm{~Hz}, 1 \mathrm{H}), 7.35-7.21(\mathrm{~m}, 5 \mathrm{H}), 7.11-7.01(\mathrm{~m}, 1 \mathrm{H}), 5.08(\mathrm{dd}, J=5.8,2.6 \mathrm{~Hz}, 1 \mathrm{H}), 3.62$ (dd, $J=15.2,5.8 \mathrm{~Hz}, 1 \mathrm{H}), 2.97(\mathrm{dd}, J=15.2,2.7 \mathrm{~Hz}, 1 \mathrm{H}) .{ }^{13} \mathrm{C} \mathrm{NMR}\left(101 \mathrm{MHz}, \mathrm{CDCl}_{3}\right) \delta 163.9,150.2$, 148.2, 137.4, 133.9, 133.2, 129.2, 124.2, 124.1, 116.7, 51.7, 46.9. HRMS exact mass calculated for $[\mathrm{M}+\mathrm{H}]^{+}\left(\mathrm{C}_{14} \mathrm{H}_{13} \mathrm{~N}_{2} \mathrm{O}^{+}\right): m / z 225.1023$, found $m / z 225.1024$.

\section{1-Phenyl-4-methyl-2-azetidinone (2p) ${ }^{23}$}

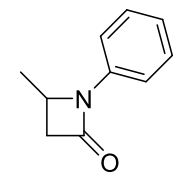

Yellow oil $(0.1 \mathrm{~g}, 62 \%)$, purified by silica gel chromatography $\left(5 \% \rightarrow 20 \%\right.$ ethyl acetate $/ n$-hexane). ${ }^{1} \mathrm{H}$ NMR (400 MHz, $\left.\mathrm{CDCl}_{3}\right) \delta 7.41-7.29(\mathrm{~m}, 4 \mathrm{H}), 7.11-7.05(\mathrm{~m}, 1 \mathrm{H}), 4.22-4.12(\mathrm{~m}, 1 \mathrm{H}), 3.24$ (dd, $J=$ 15.0, $5.4 \mathrm{~Hz}, 1 \mathrm{H}), 2.68(\mathrm{dd}, J=15.0,2.5 \mathrm{~Hz}, 1 \mathrm{H}), 1.52(\mathrm{~d}, J=6.1 \mathrm{~Hz}, 3 \mathrm{H}) .{ }^{13} \mathrm{C} \mathrm{NMR}\left(101 \mathrm{MHz}, \mathrm{CDCl}_{3}\right)$ $\delta 164.3,137.7,129.2,123.7,116.8,47.1,44.0,18.7$. HRMS exact mass calculated for $[\mathrm{M}+\mathrm{Na}]^{+}$ $\left(\mathrm{C}_{10} \mathrm{H}_{11} \mathrm{NONa}^{+}\right): \mathrm{m} / z$ 184.0733, found $\mathrm{m} / z$ 184.0731. 


\section{1-Phenyl-4-cyclohexyl-2-azetidinone (2q)}

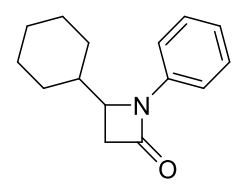

Light yellow solid $(0.165 \mathrm{~g}, 72 \%)$, purified by silica gel chromatography ( $5 \% \rightarrow 20 \%$ ethyl acetate $/ n$ hexane). ${ }^{1} \mathrm{H}$ NMR $\left(400 \mathrm{MHz}, \mathrm{CDCl}_{3}\right) \delta 7.42-7.27(\mathrm{~m}, 4 \mathrm{H}), 7.10-7.04(\mathrm{~m}, 1 \mathrm{H}), 4.04(\mathrm{td}, J=5.3,2.7$ $\mathrm{Hz}, 1 \mathrm{H}), 2.98$ (dd, $J=15.2,5.6 \mathrm{~Hz}, 1 \mathrm{H}), 2.83(\mathrm{dd}, J=15.2,2.7 \mathrm{~Hz}, 1 \mathrm{H}), 2.07-1.92(\mathrm{~m}, 1 \mathrm{H}), 1.81-$ $1.55(\mathrm{~m}, 5 \mathrm{H}), 1.33-0.97(\mathrm{~m}, 5 \mathrm{H}) .{ }^{13} \mathrm{C}$ NMR $\left(101 \mathrm{MHz}, \mathrm{CDCl}_{3}\right) \delta 165.0,138.1,129.1,123.8,117.4$, $55.4,38.4,38.2,29.6,29.6,26.3,26.1,25.6,25.2$. HRMS exact mass calculated for $[\mathrm{M}+\mathrm{Na}]^{+}$ $\left(\mathrm{C}_{15} \mathrm{H}_{19} \mathrm{NONa}^{+}\right): m / z 252.1359$, found $\mathrm{m} / z 252.1359$.

Cis-1,3,4-triphenyl-2-azetidinone (cis-2t) ${ }^{24}$<smiles>O=C1c2ccccc2C(c2ccccc2)C(=O)N1c1ccccc1</smiles>

White solid ( $0.175 \mathrm{~g}, 58.5 \%)$, purified by silica gel chromatography ( $5 \% \rightarrow 20 \%$ ethyl acetate $/ n$-hexane). ${ }^{1} \mathrm{H}$ NMR $\left(400 \mathrm{MHz}, \mathrm{CDCl}_{3}\right) \delta 7.48-7.42(\mathrm{~m}, 2 \mathrm{H}), 7.33-7.27(\mathrm{~m}, 2 \mathrm{H}), 7.15-7.03(\mathrm{~m}, 11 \mathrm{H}), 5.48(\mathrm{~d}$, $J=6.1 \mathrm{~Hz}, 1 \mathrm{H}), 5.02(\mathrm{~d}, J=6.1 \mathrm{~Hz}, 1 \mathrm{H}) .{ }^{13} \mathrm{C} \mathrm{NMR}\left(101 \mathrm{MHz}, \mathrm{CDCl}_{3}\right) \delta 165.7,137.8,134.4,132.2$, $129.2,129.0,128.3,128.2,127.9,127.2,127.2,124.1,117.3,60.4,60.3$. HRMS exact mass calculated for $[\mathrm{M}+\mathrm{Na}]^{+}\left(\mathrm{C}_{21} \mathrm{H}_{17} \mathrm{NONa}^{+}\right): m / z 322.1202$, found $m / z 322.1202$.

Trans-1,3,4-triphenyl-2-azetidinone (trans-2t) ${ }^{24}$<smiles>O=C1c2ccccc2C(c2ccccc2)C(=O)N1c1ccccc1</smiles>

White solid $(0.073 \mathrm{~g}, 24.5 \%)$, purified by silica gel chromatography $(5 \% \rightarrow 20 \%$ ethyl acetate $/ n$ hexane). ${ }^{1} \mathrm{H}$ NMR $\left(400 \mathrm{MHz}, \mathrm{CDCl}_{3}\right) \delta 7.42-7.22(\mathrm{~m}, 14 \mathrm{H}), 7.09-7.03(\mathrm{~m}, 1 \mathrm{H}), 4.95(\mathrm{~d}, J=2.6 \mathrm{~Hz}$, $1 \mathrm{H}), 4.28(\mathrm{~d}, J=2.6 \mathrm{~Hz}, 1 \mathrm{H}) .{ }^{13} \mathrm{C} \mathrm{NMR}\left(101 \mathrm{MHz}, \mathrm{CDCl}_{3}\right) \delta 165.7,137.6,137.5,134.8,129.4,129.2$, $129.1,128.7,128.0,127.5,125.9,124.1,117.3,65.2,63.7$. HRMS exact mass calculated for $[\mathrm{M}+\mathrm{Na}]^{+}$ $\left(\mathrm{C}_{21} \mathrm{H}_{17} \mathrm{NONa}^{+}\right): \mathrm{m} / \mathrm{z} 322.1202$, found $\mathrm{m} / \mathrm{z} 322.1199$. 


\section{${ }^{1} \mathrm{H}$ and ${ }^{13} \mathrm{C}$ Spectra}

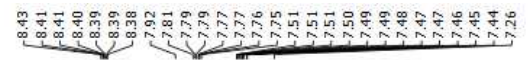

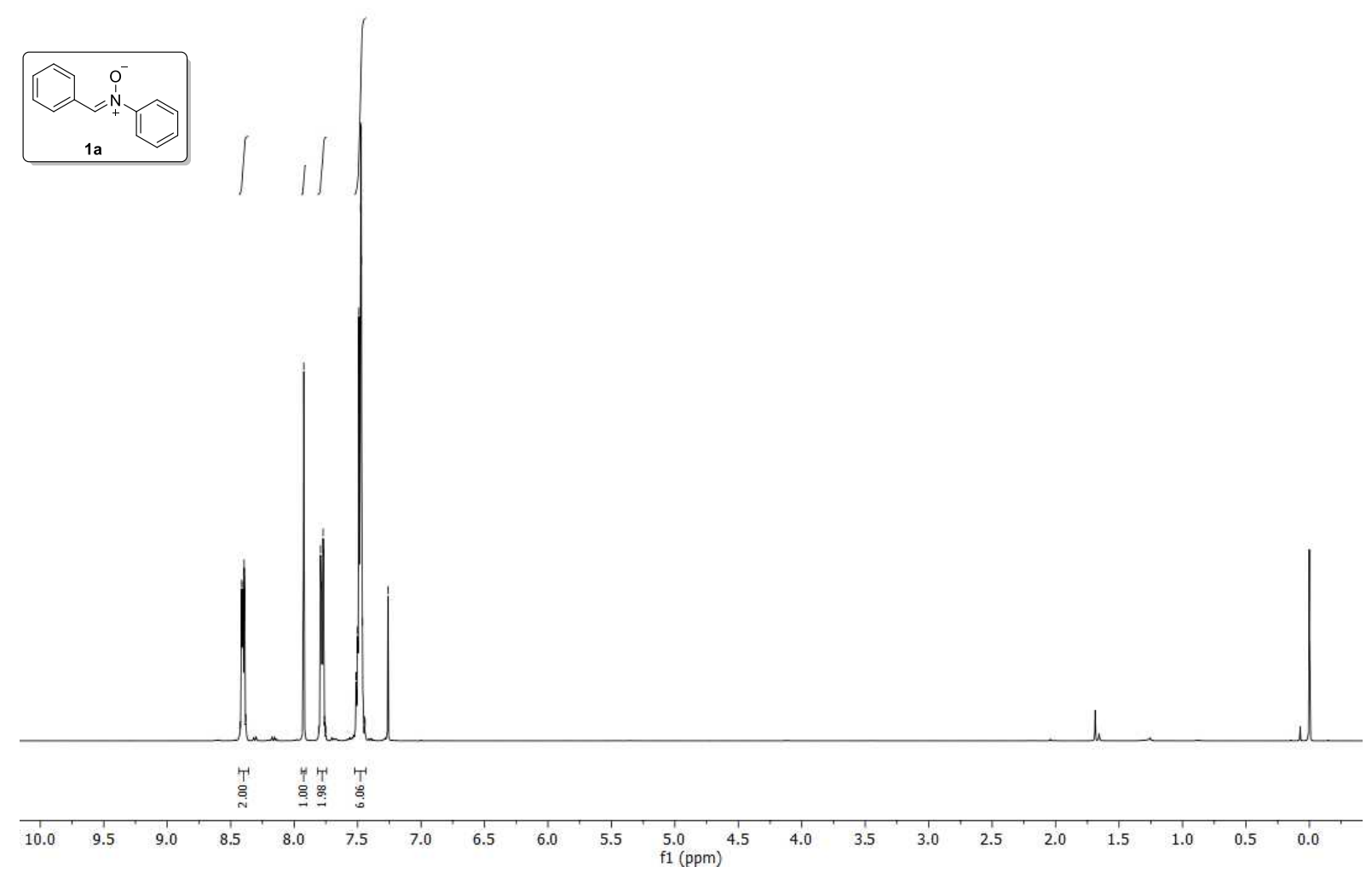

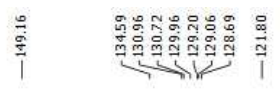

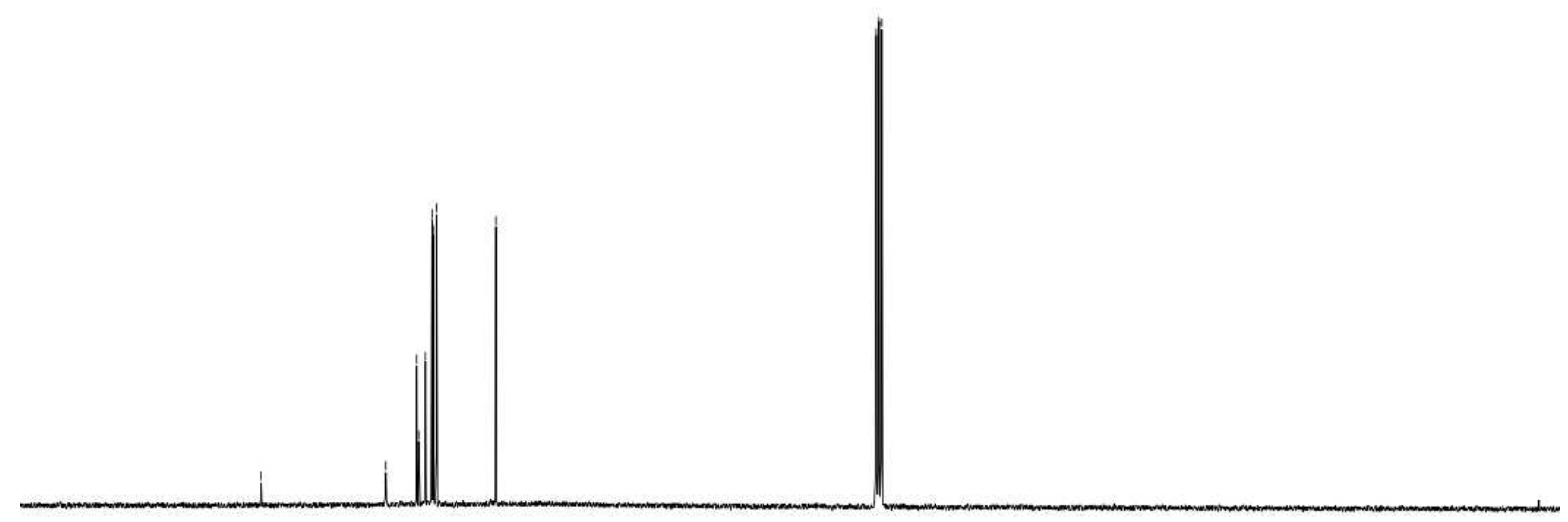

\begin{tabular}{|c|c|c|c|c|c|c|c|c|c|c|c|c|c|c|c|c|}
\hline 170 & 160 & 150 & 140 & 130 & 120 & 110 & 100 & $\begin{array}{l}90 \\
\mathrm{f} 1(\mathrm{ppm})\end{array}$ & 80 & 70 & 60 & 50 & 40 & 30 & 20 & 10 \\
\hline
\end{tabular}



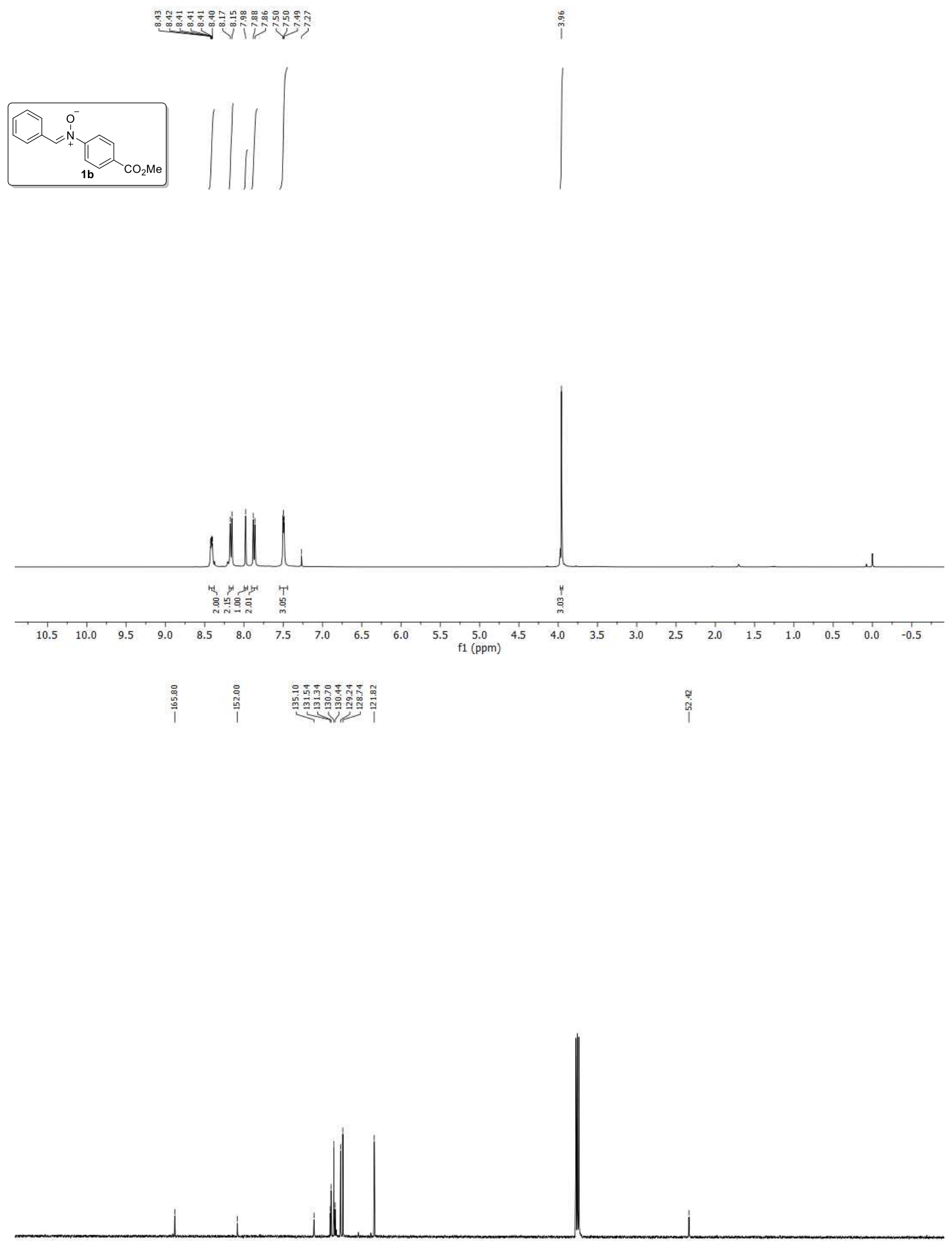

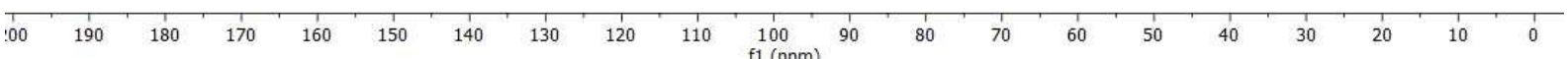




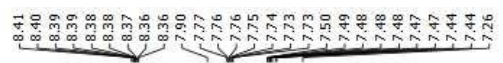

(1)

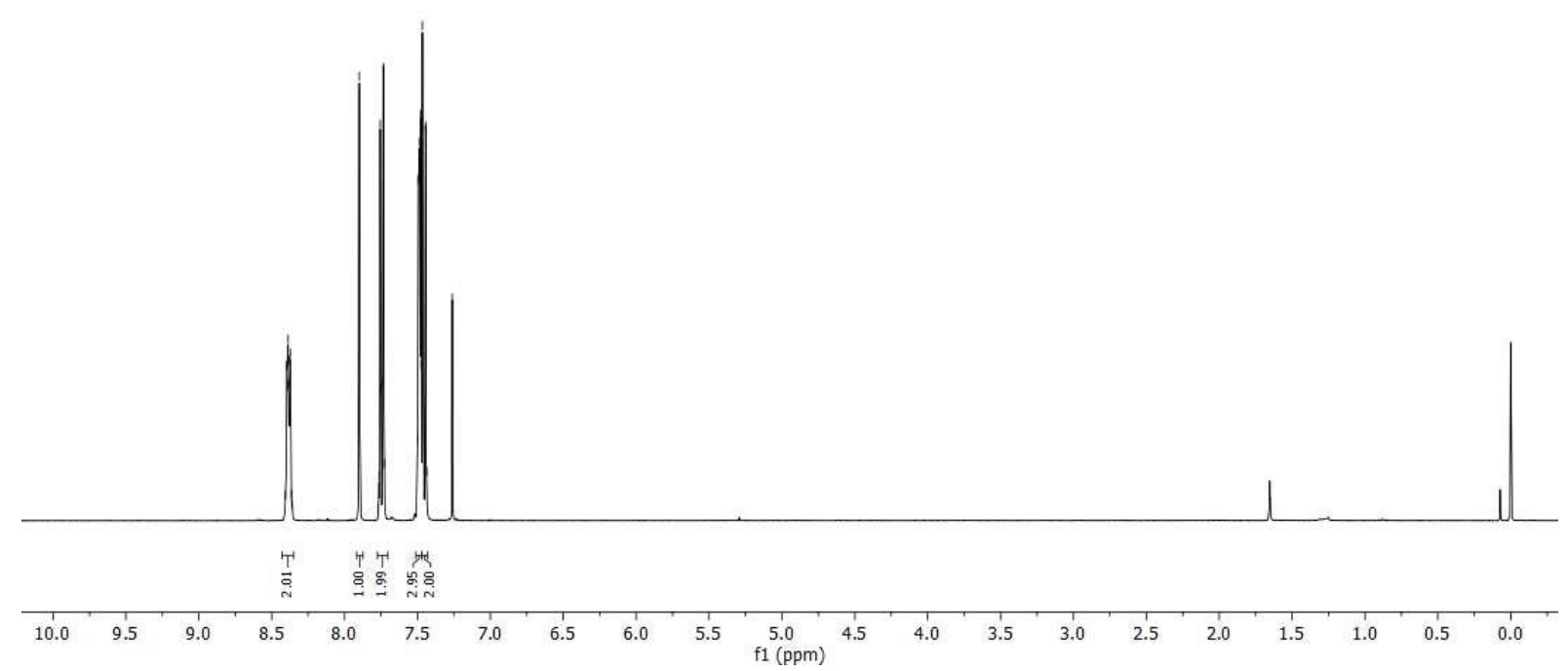

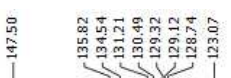

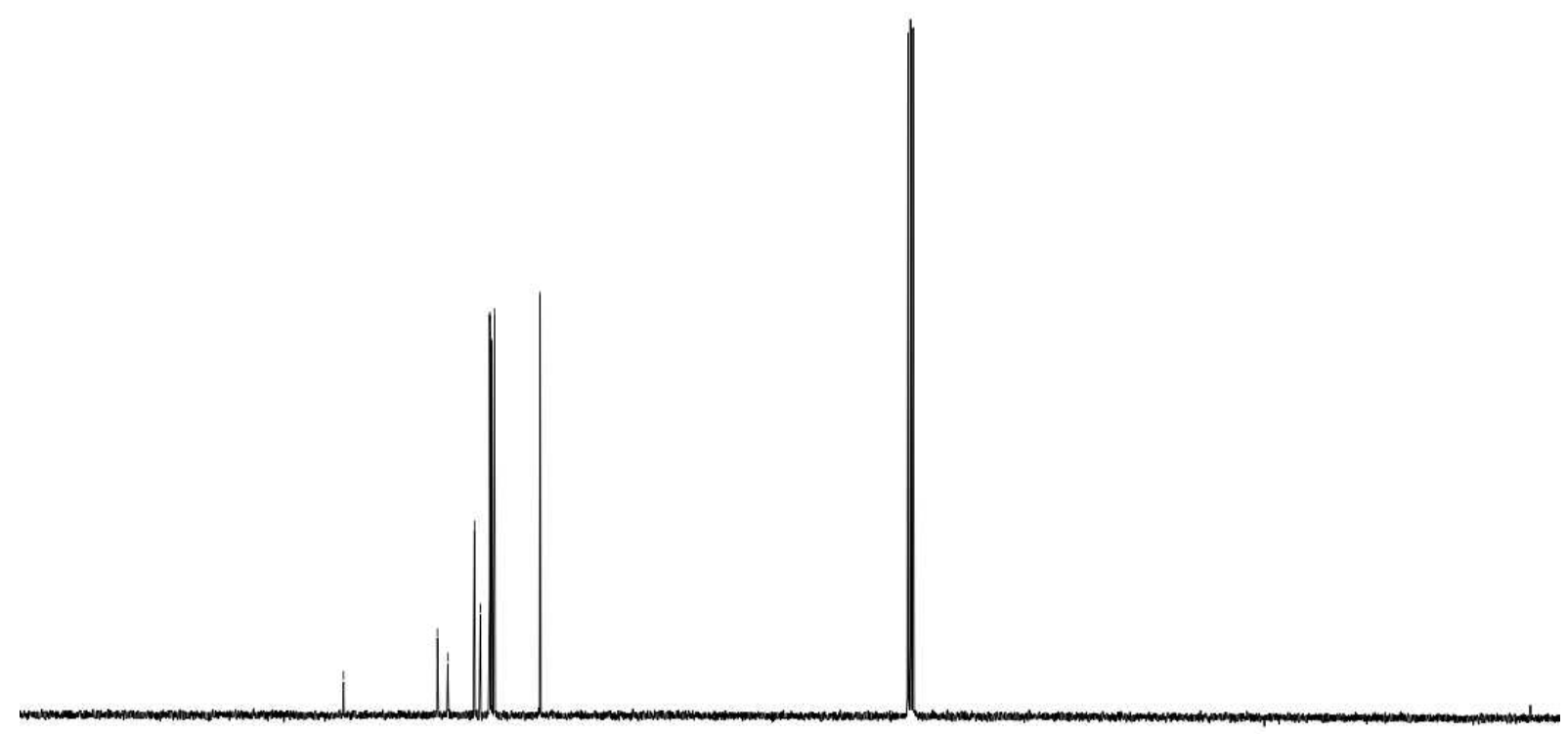

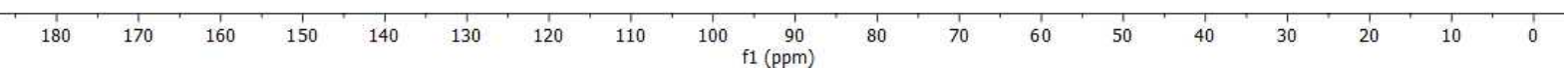




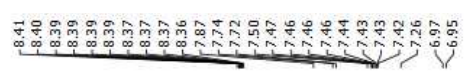
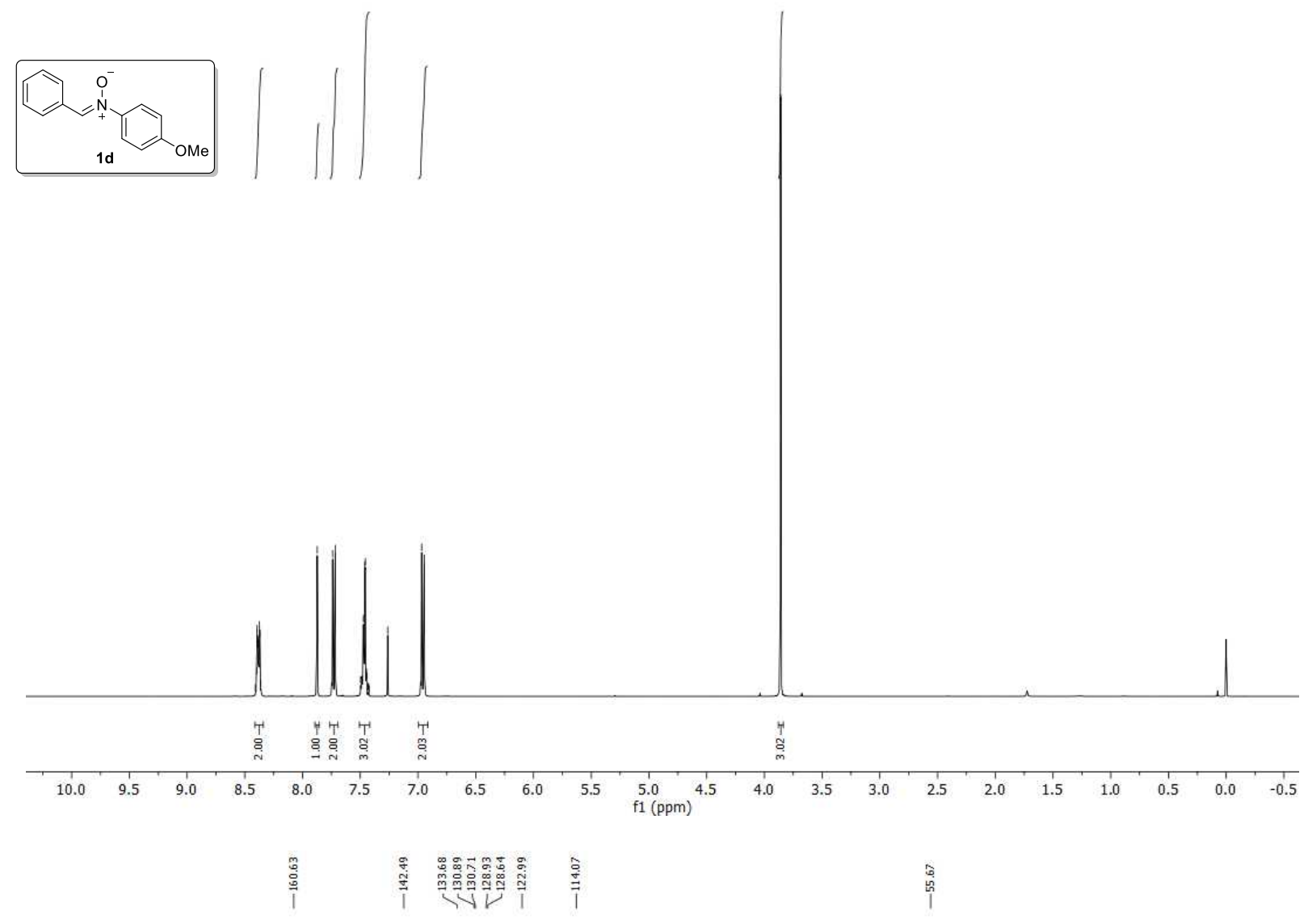
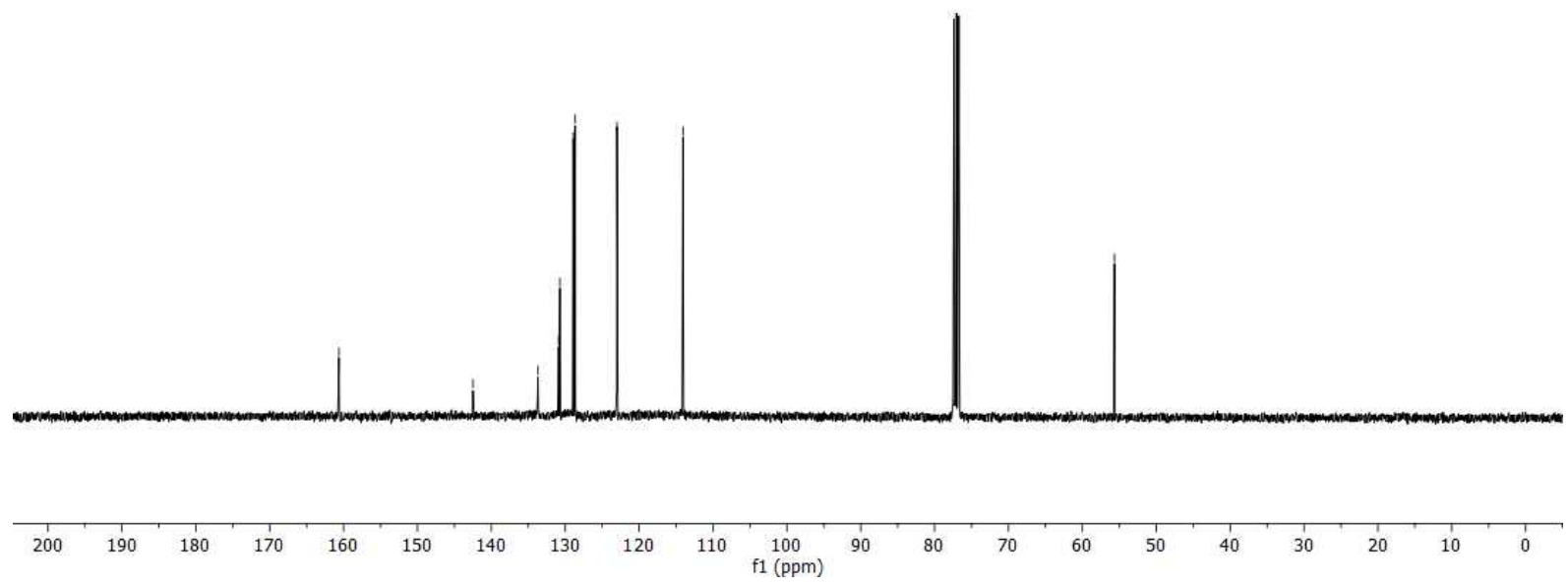

S18 


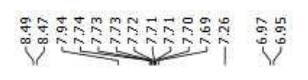

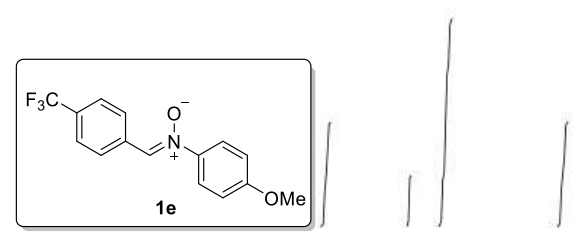

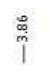

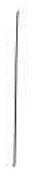

.

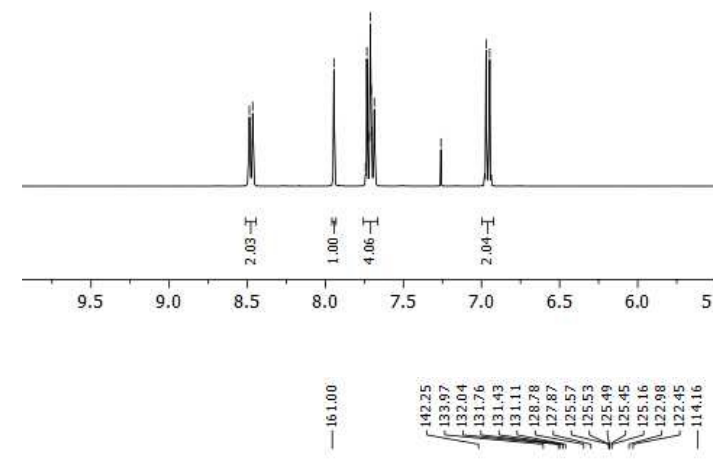

i)
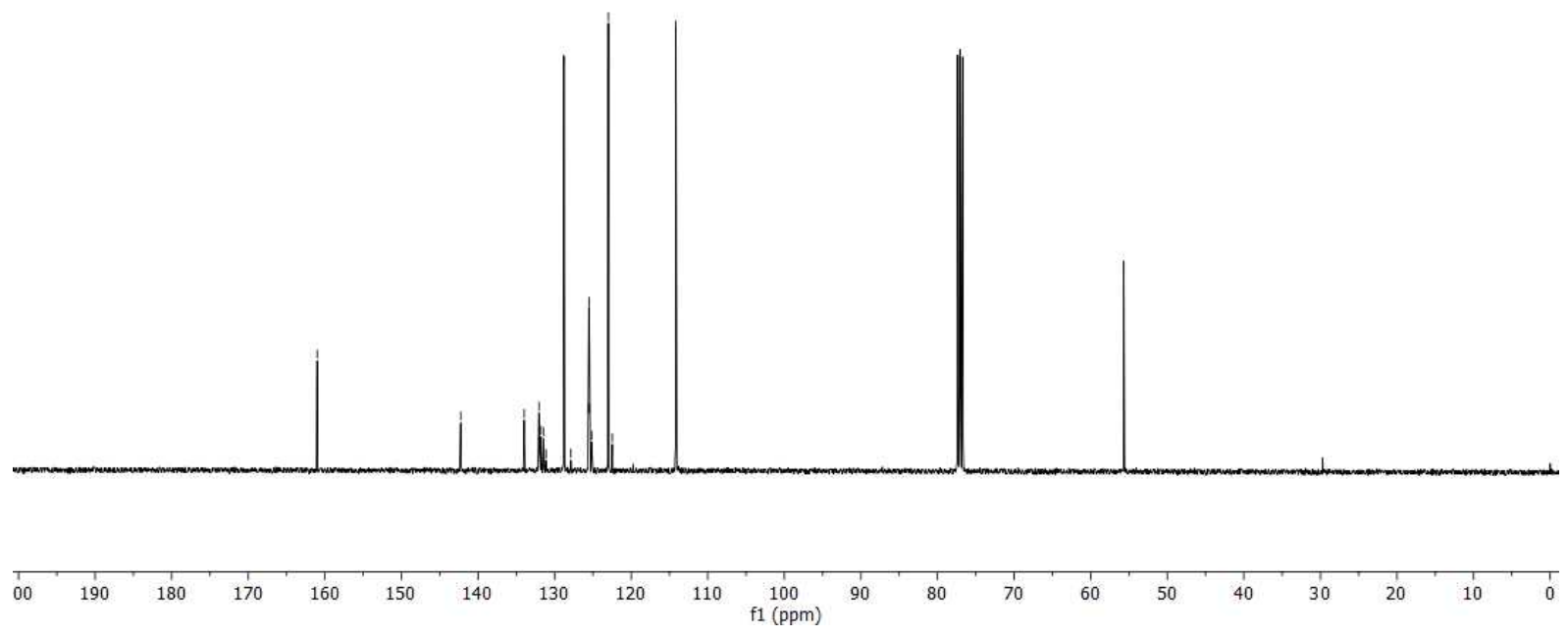

S19 


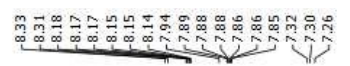
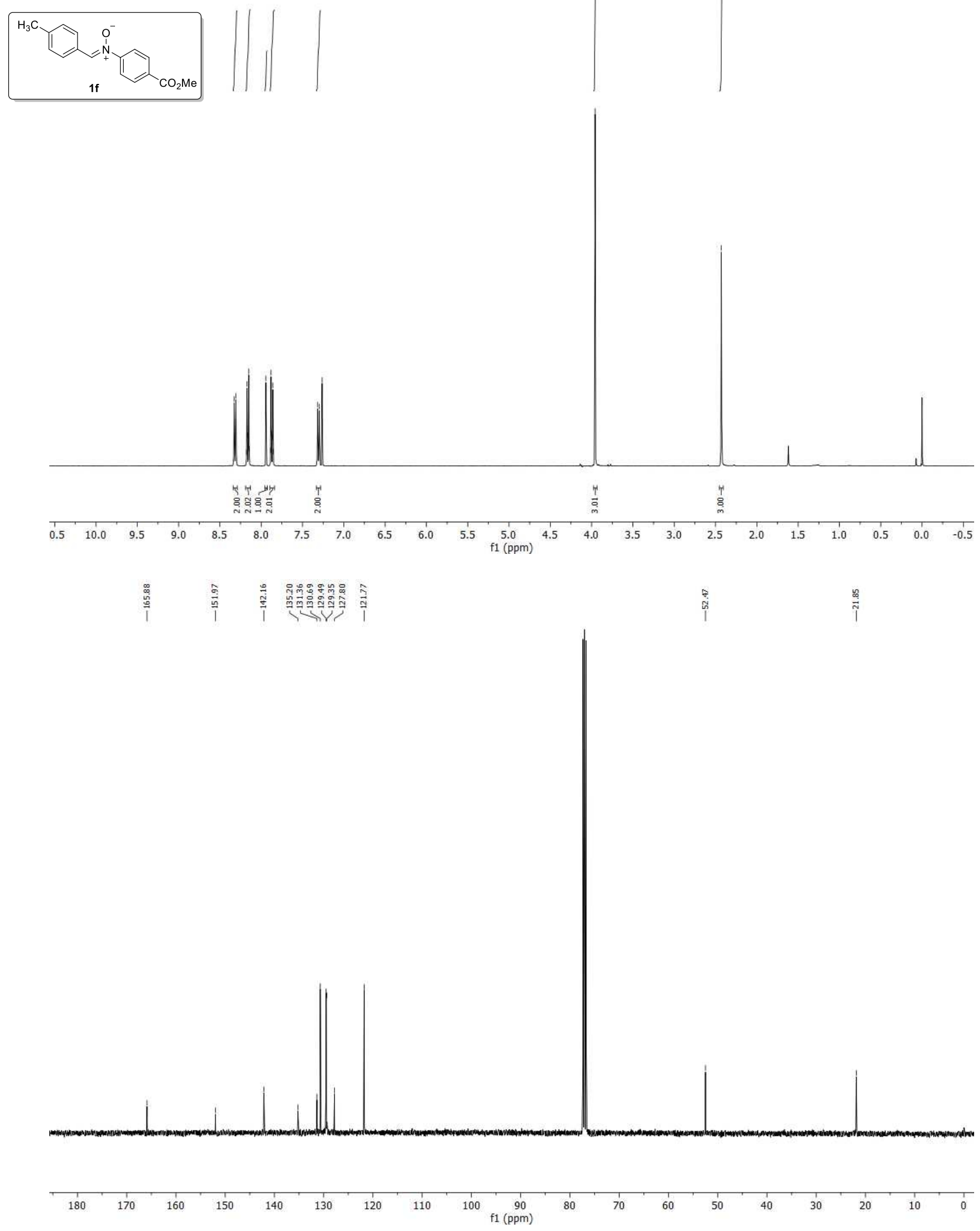

S20 

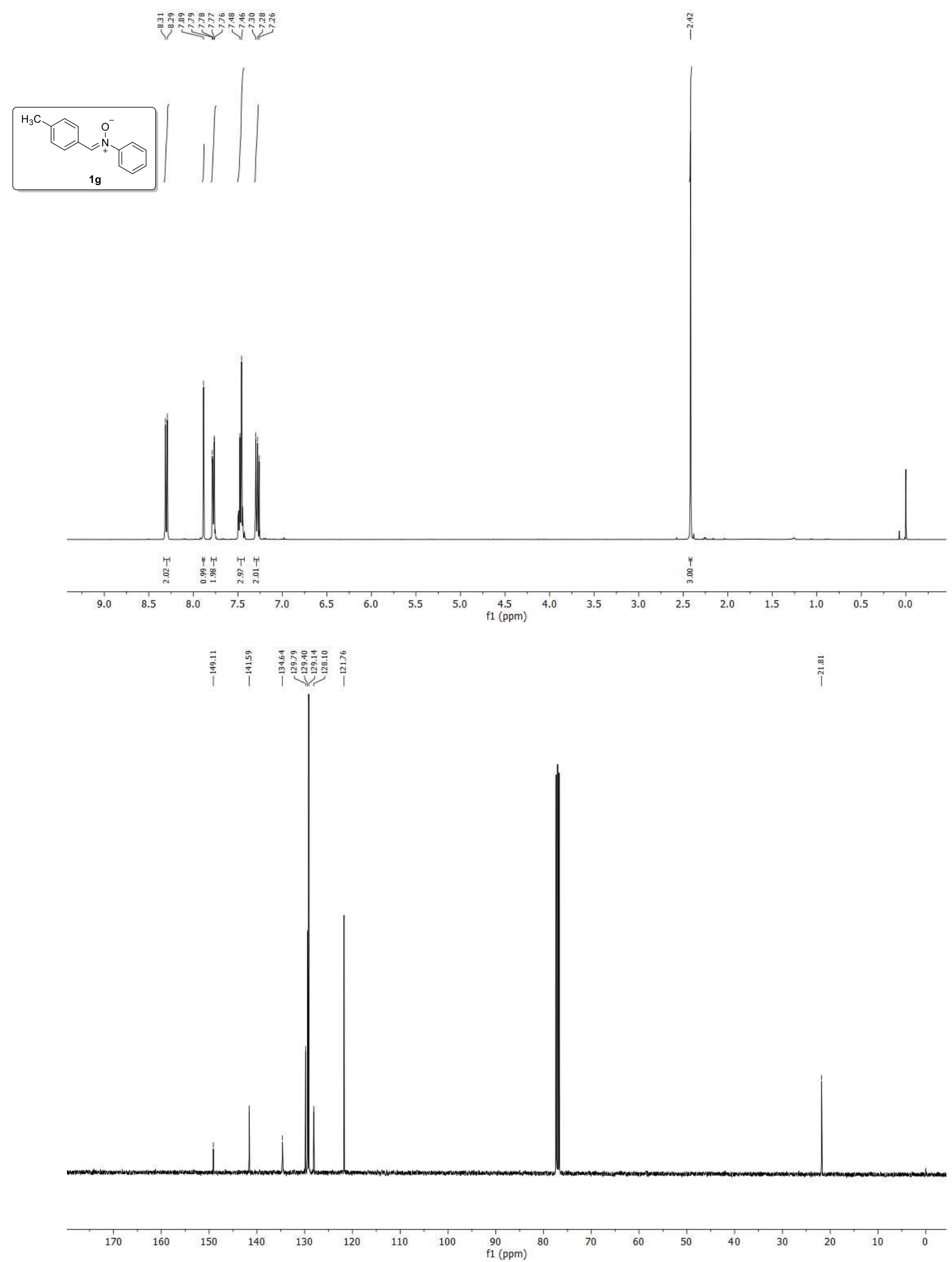


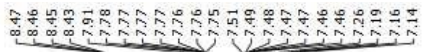
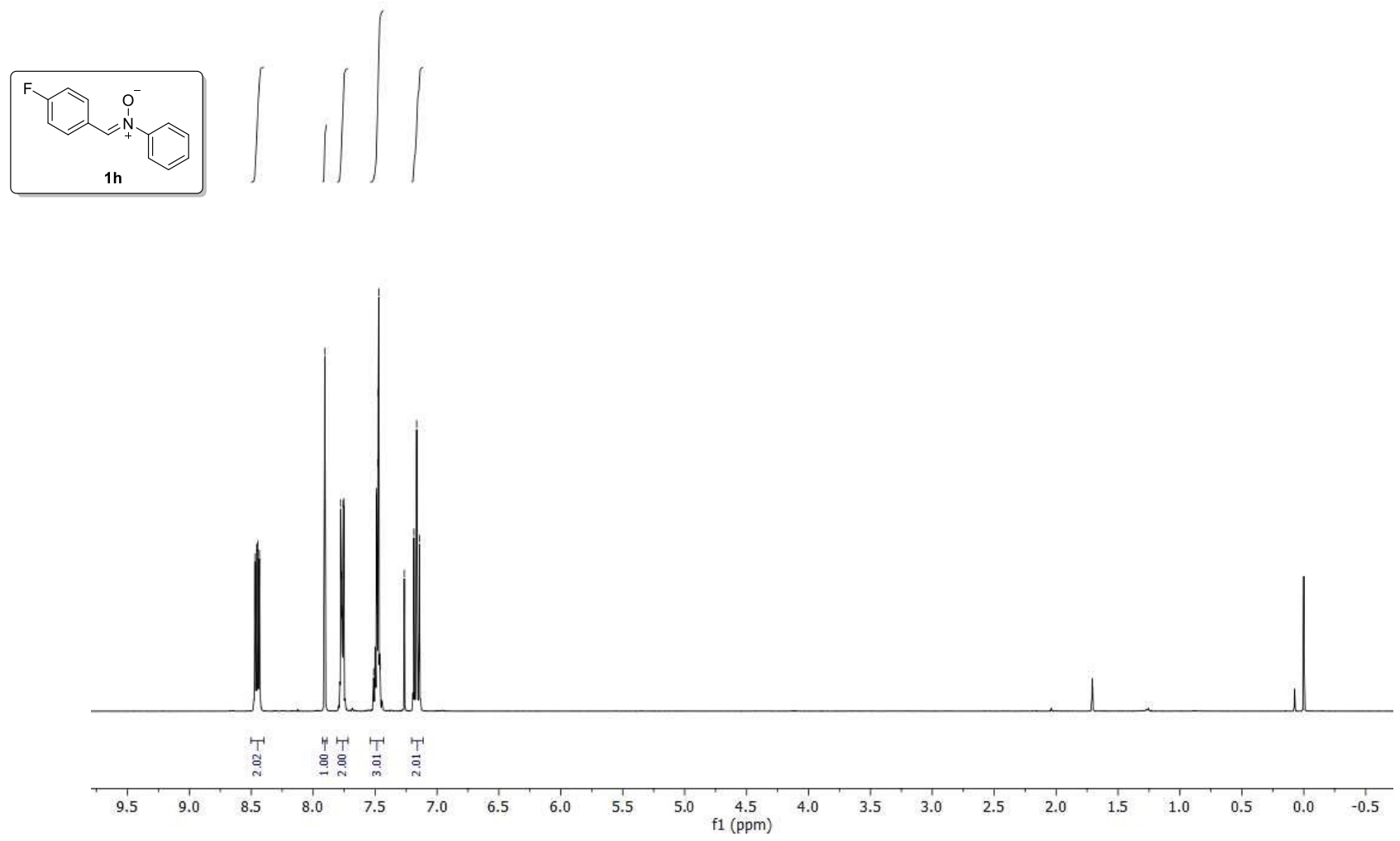

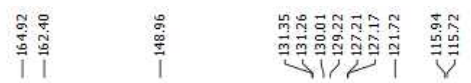
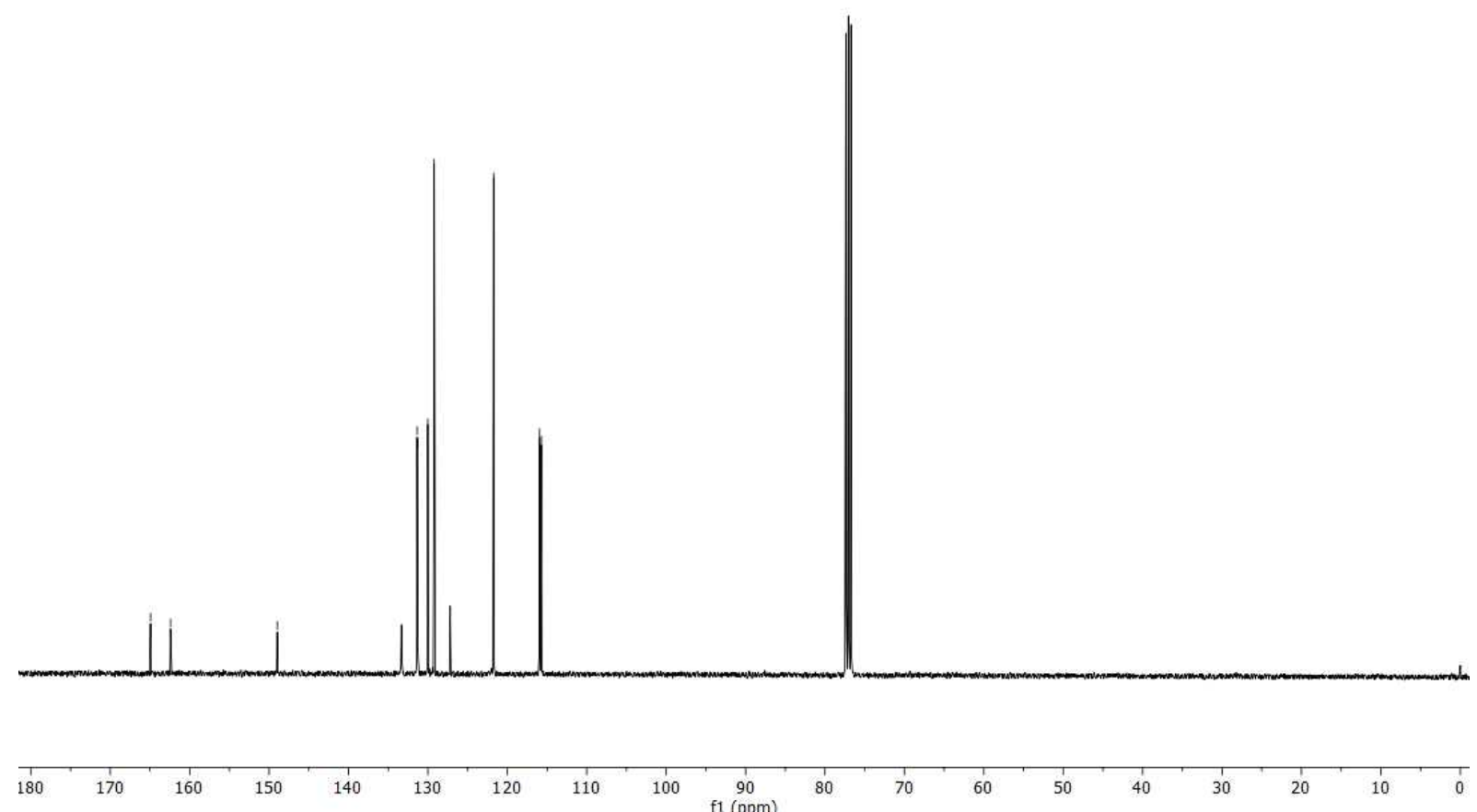


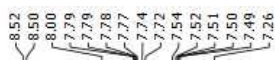
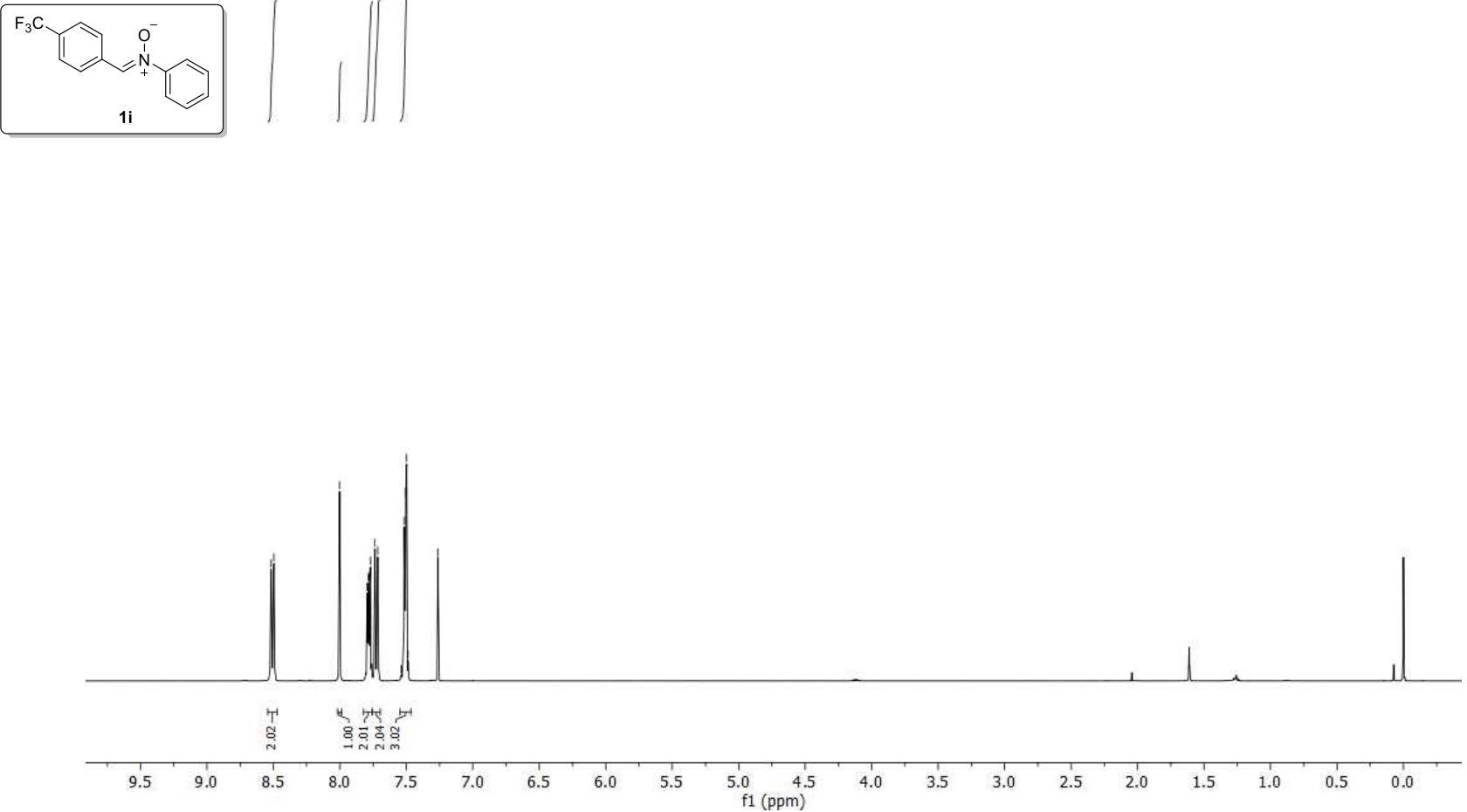

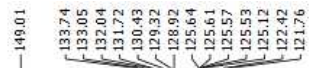

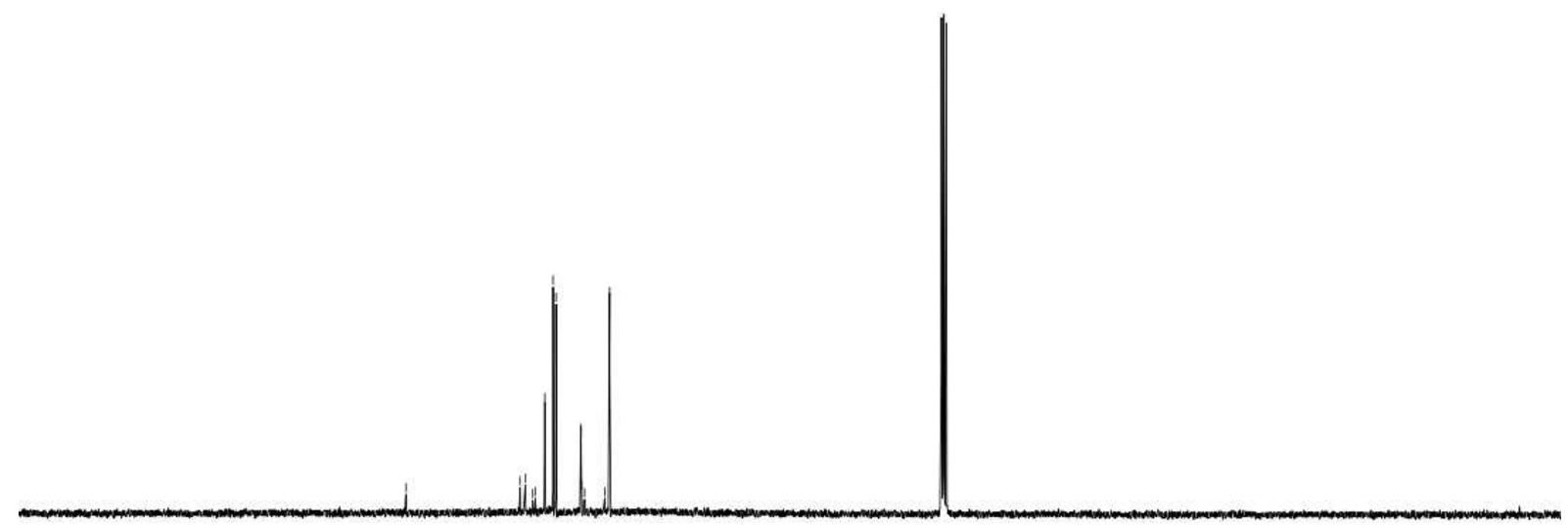

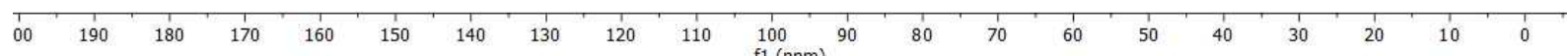



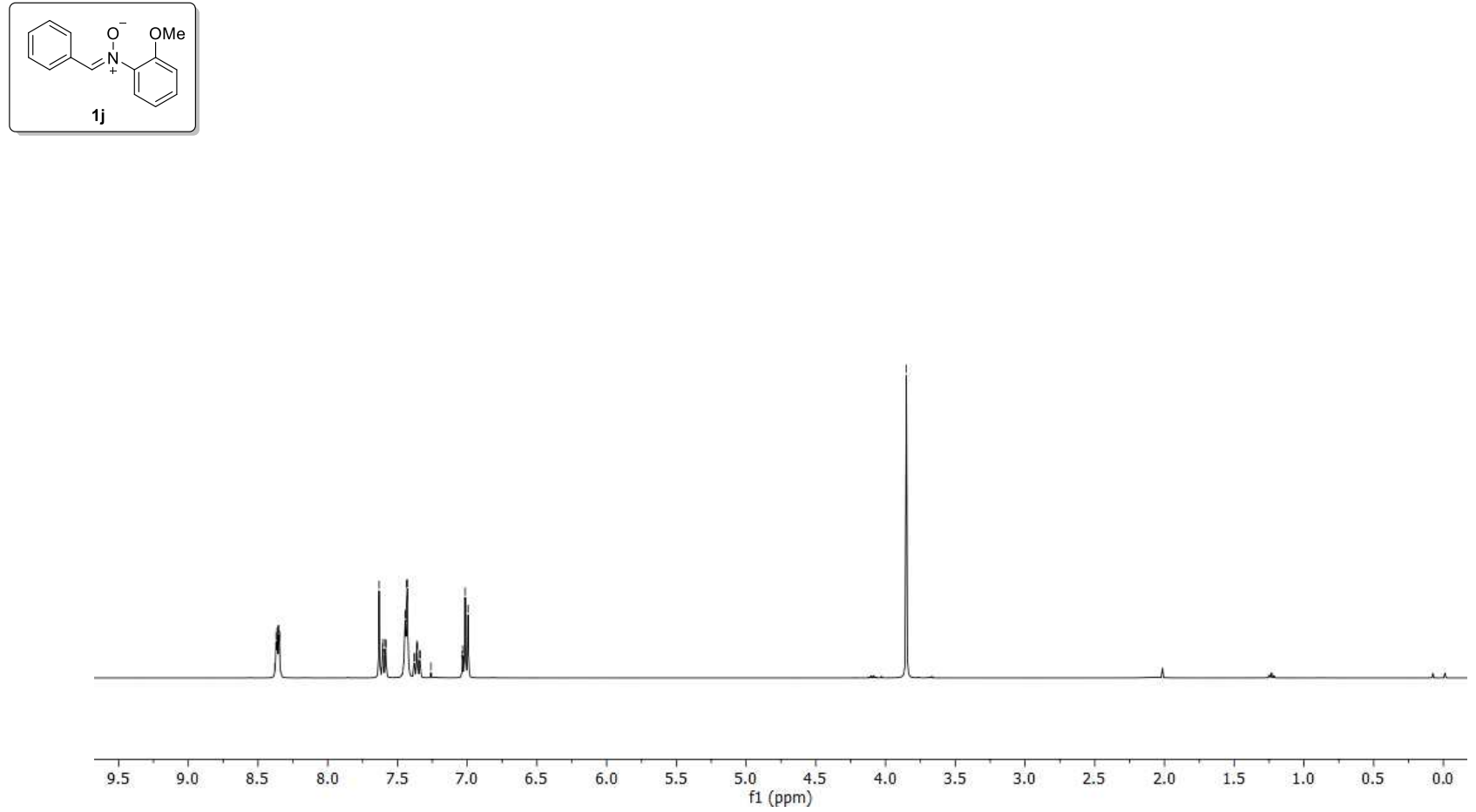

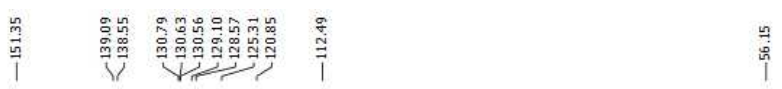

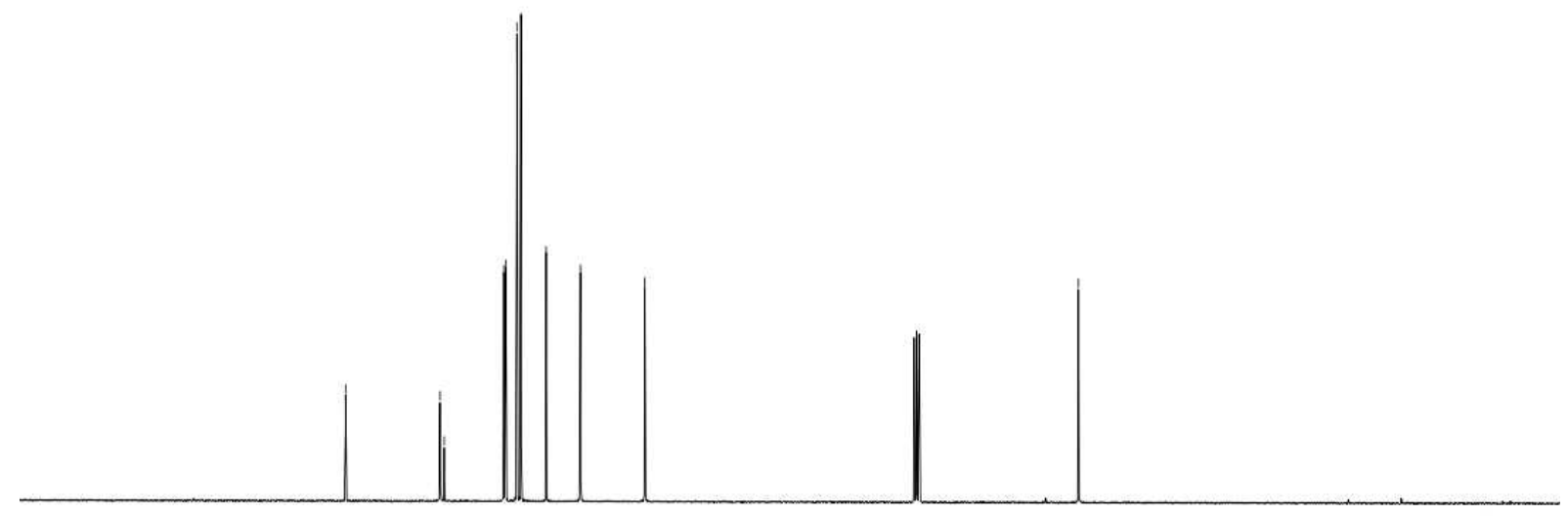

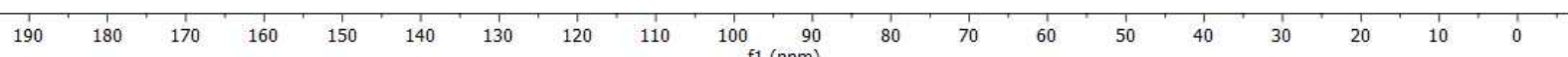




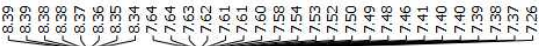
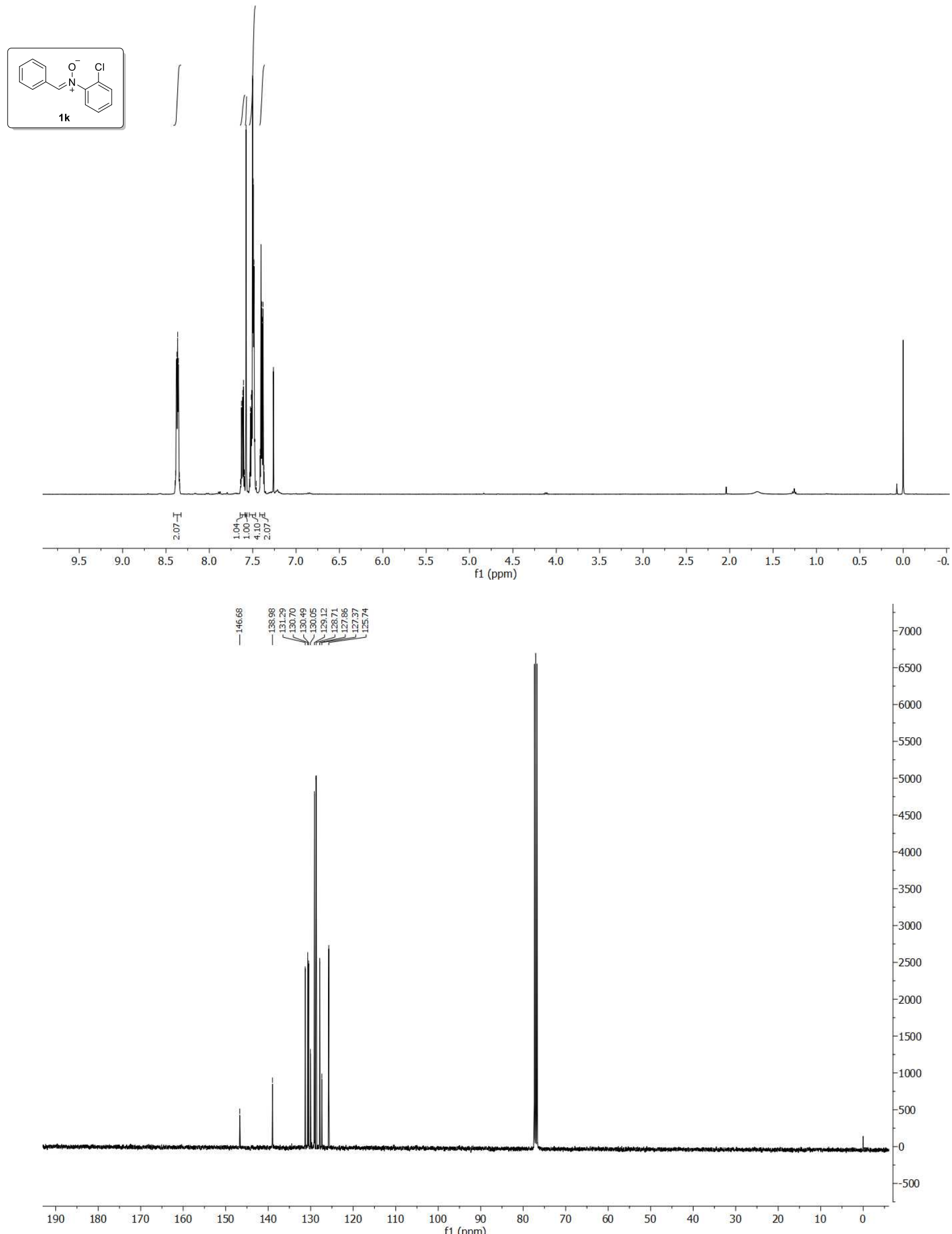


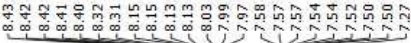
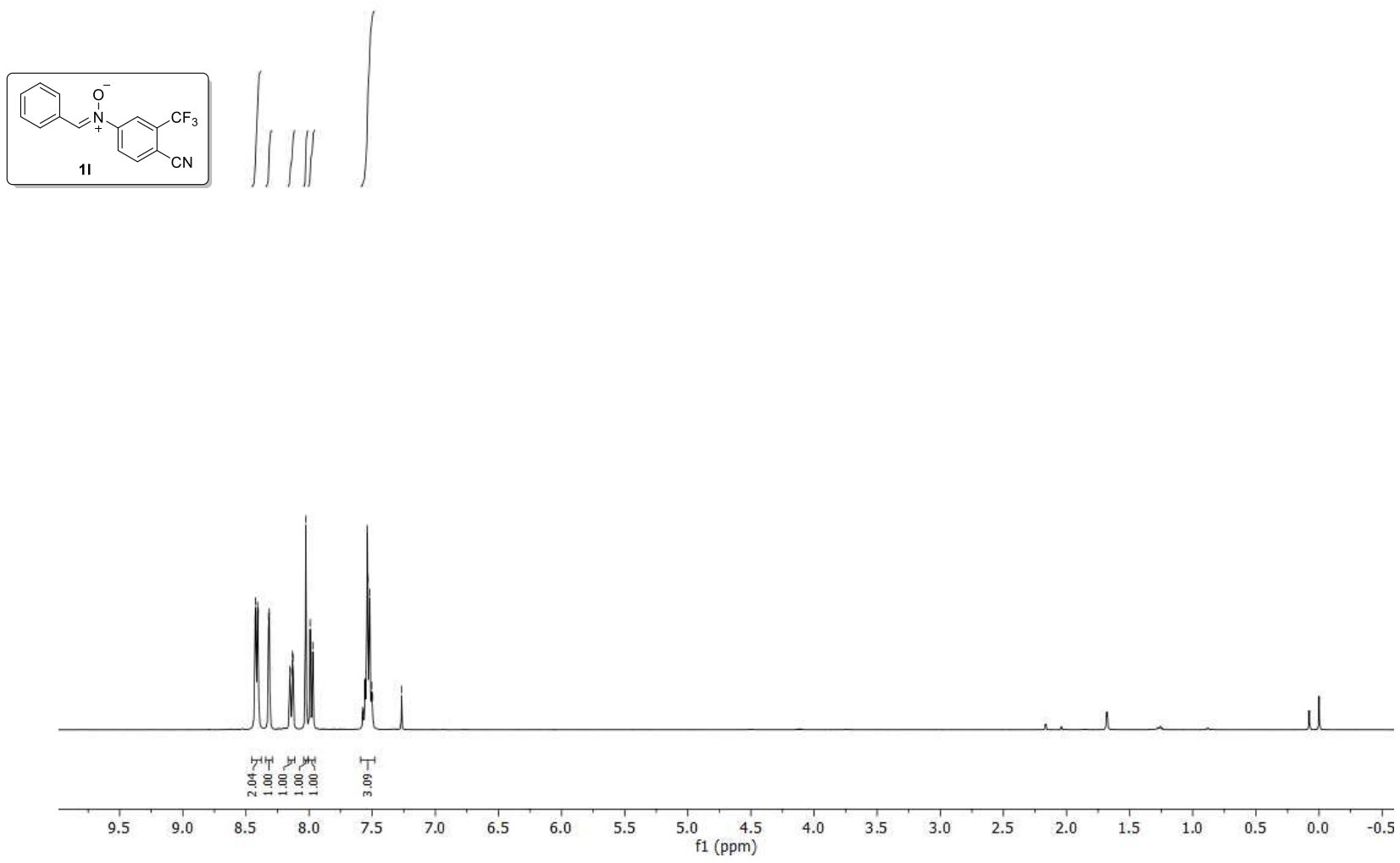

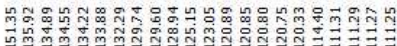

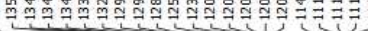
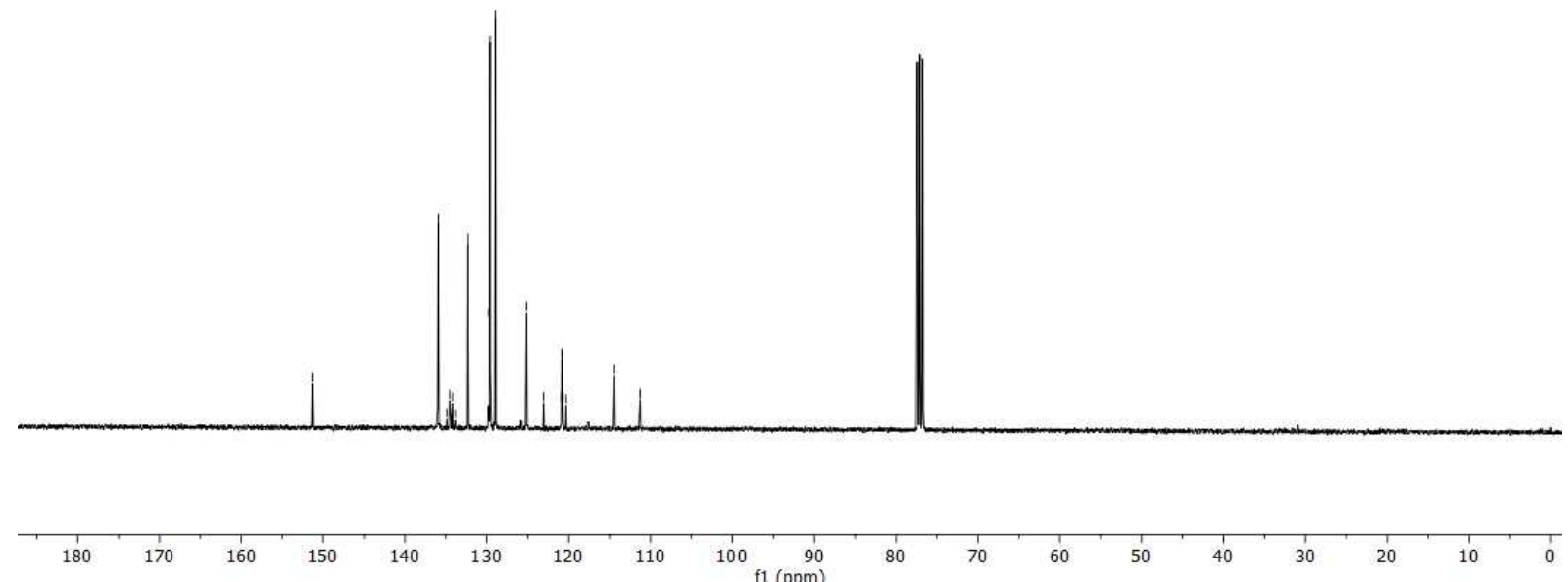


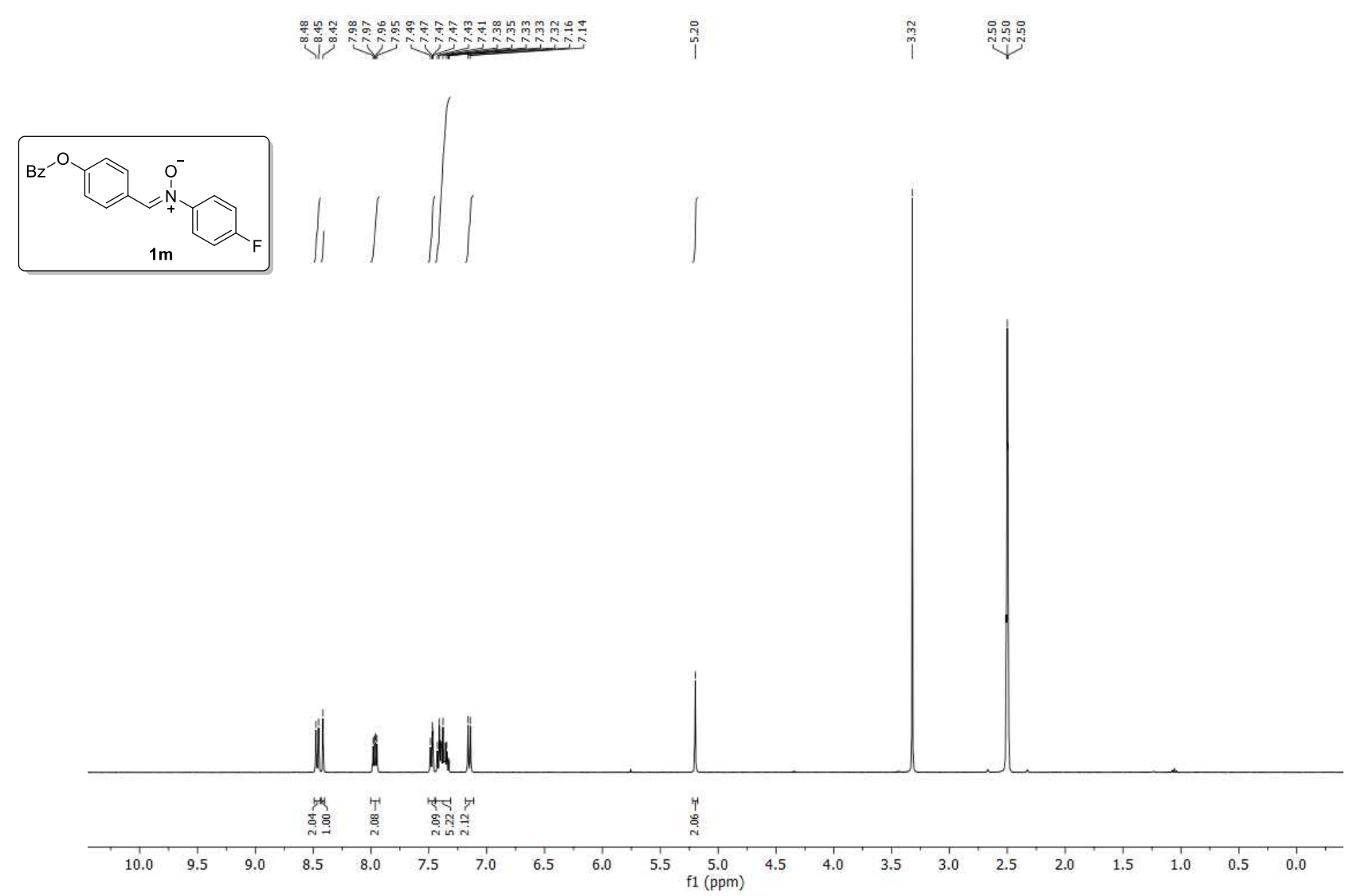

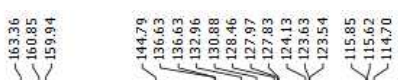

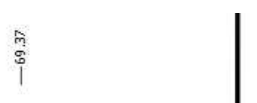

\begin{tabular}{llllllllllllllllllllll}
\hline 10 & 200 & 190 & 180 & 170 & 160 & 150 & 140 & 130 & 120 & 110 & 100 & 90 & 80 & 70 & 60 & 50 & 40 & 30 & 20 & 10 & 0
\end{tabular} $1(\mathrm{ppm})$ 


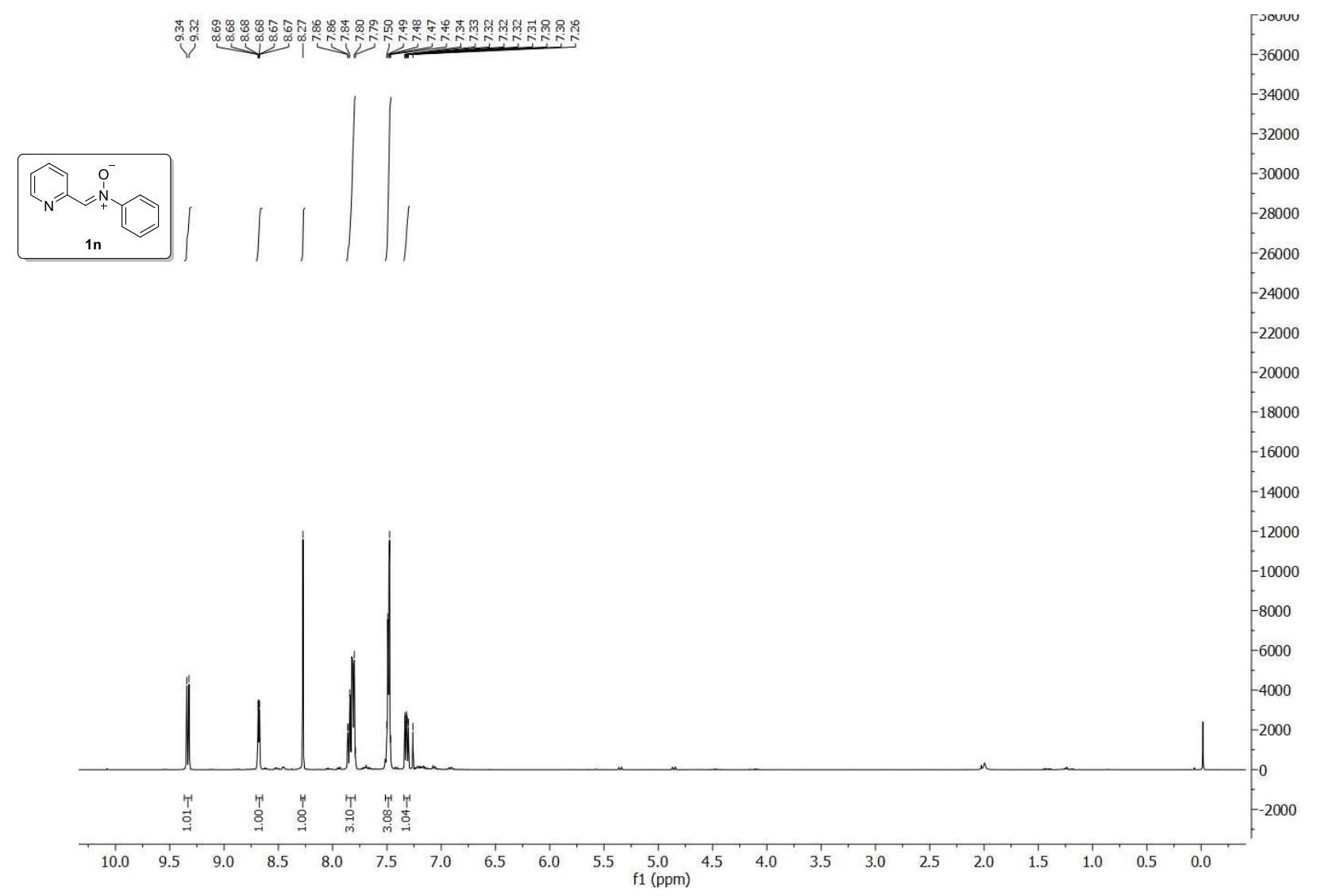

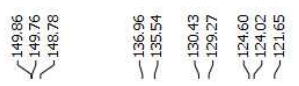
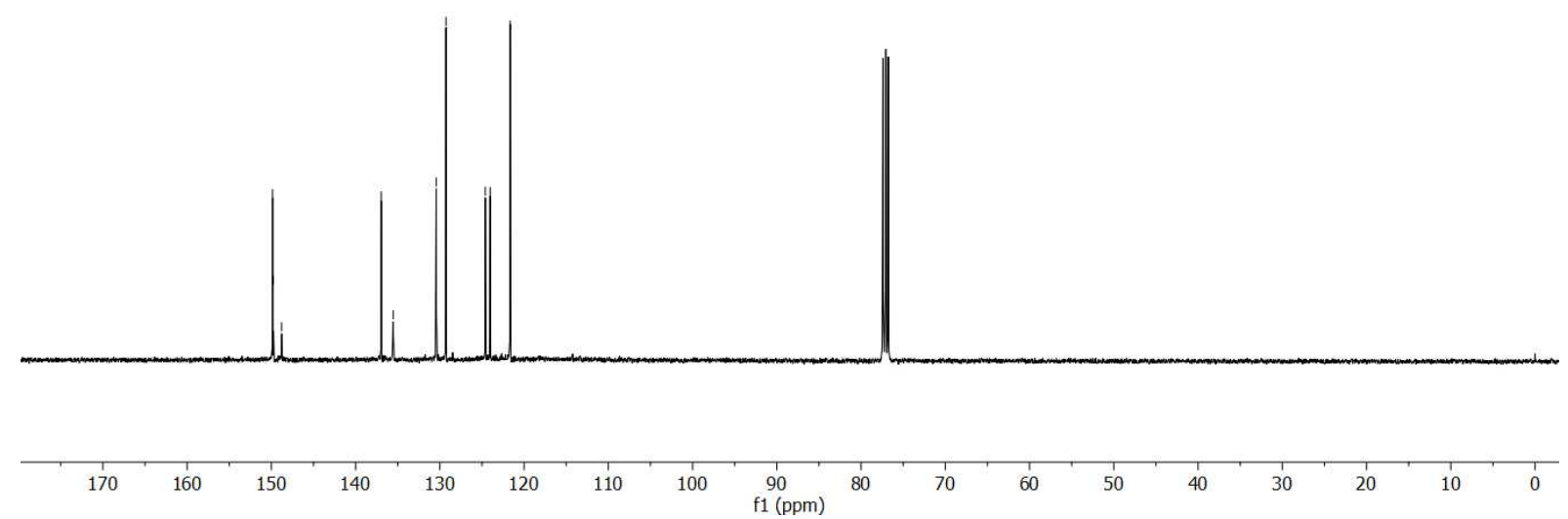


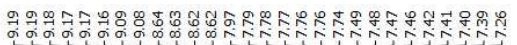
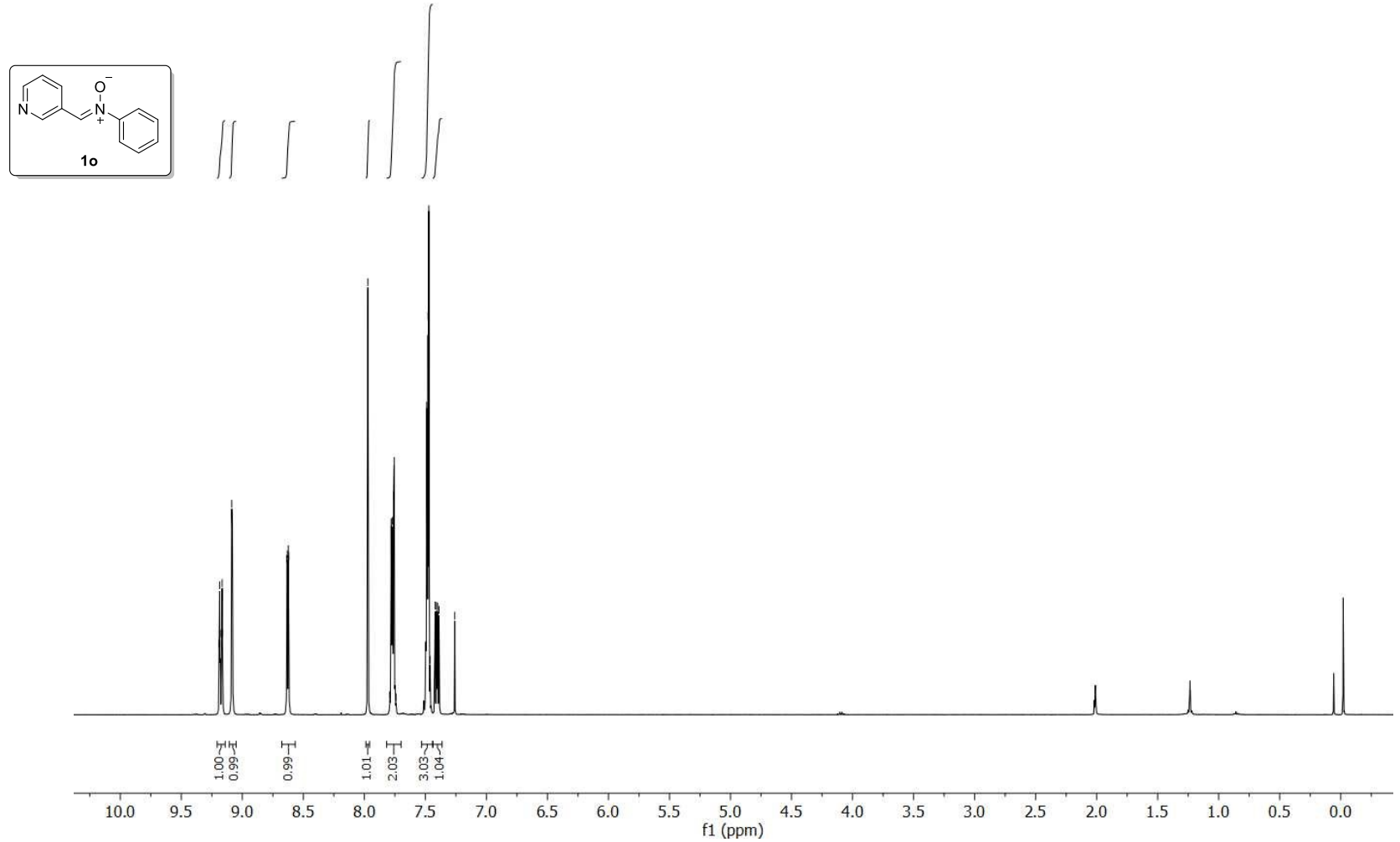

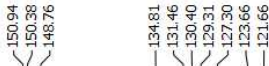
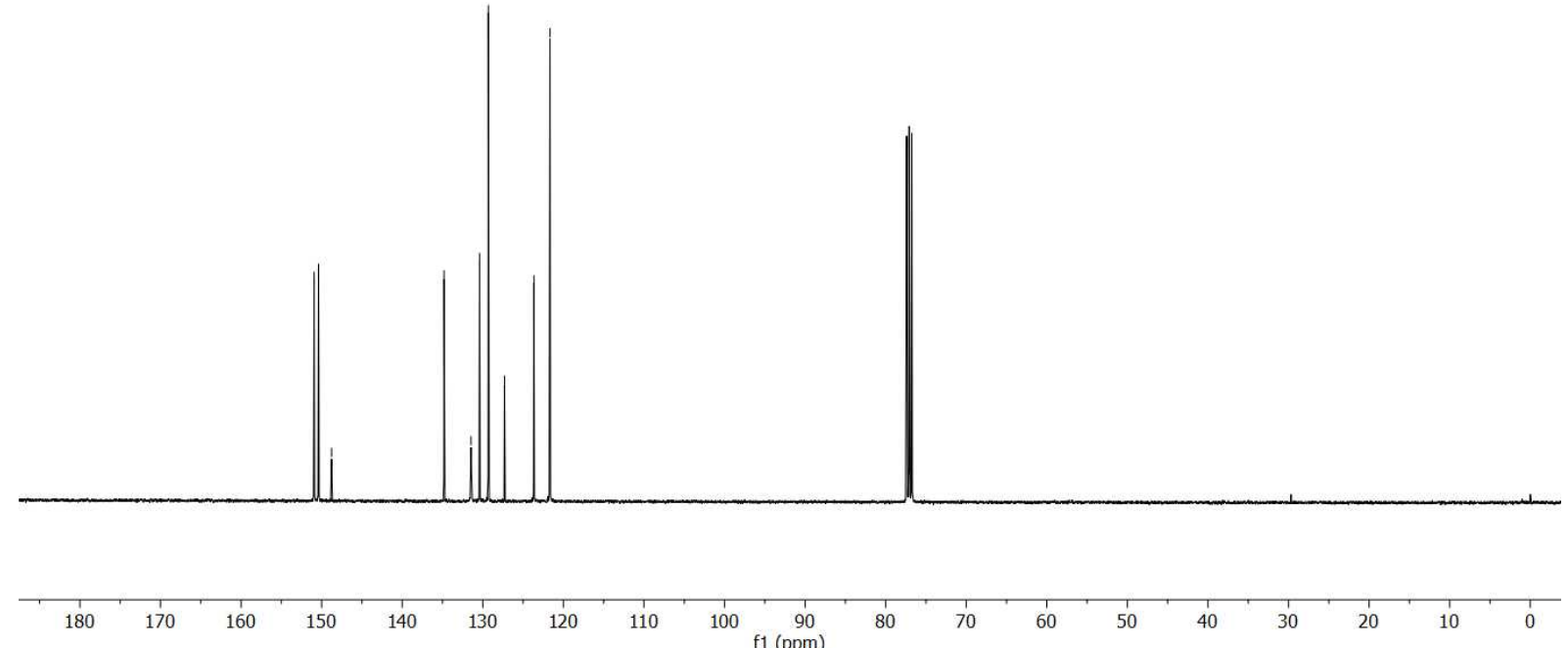


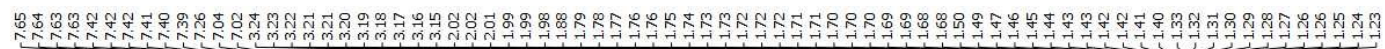
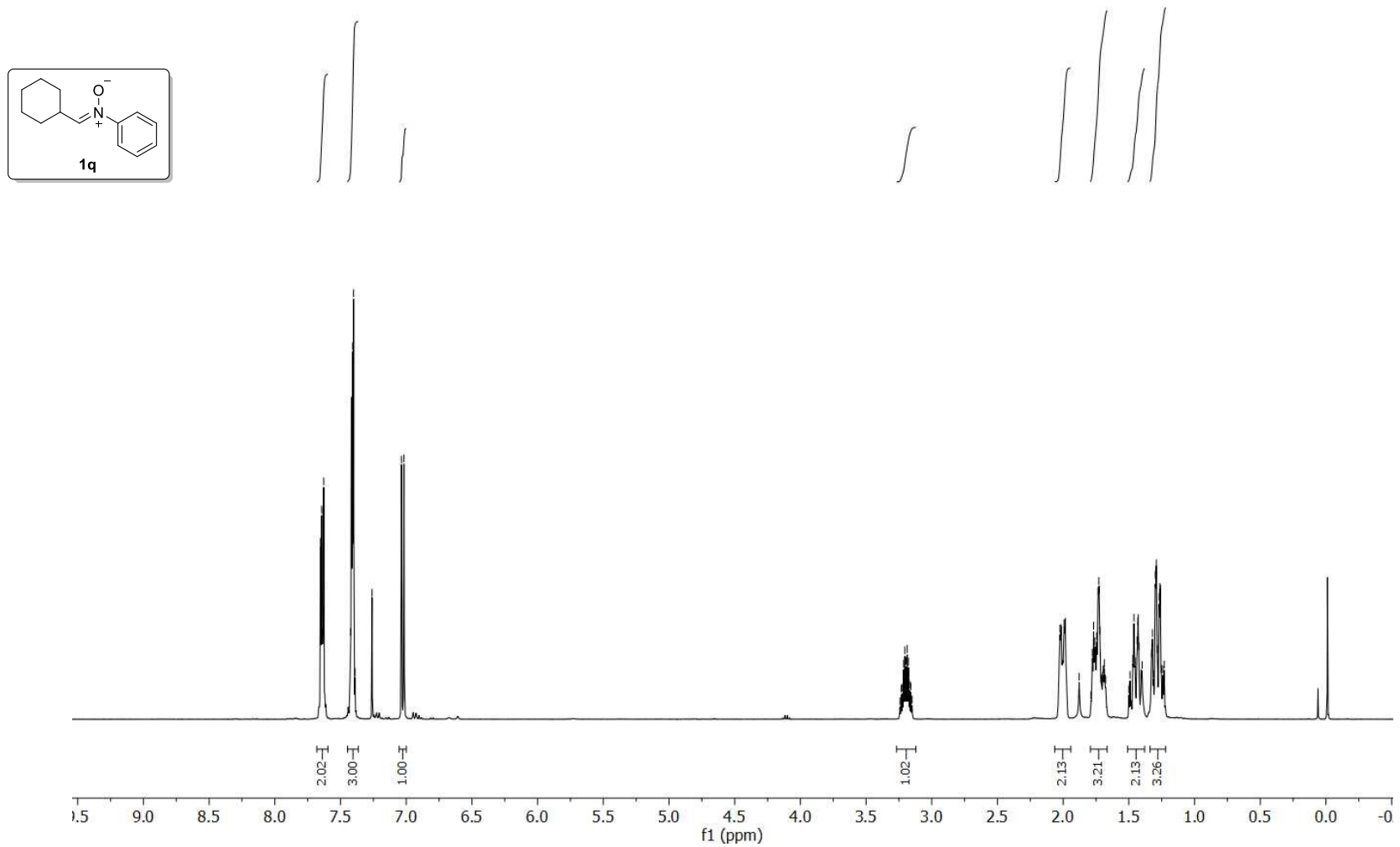

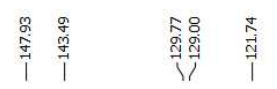

ำ
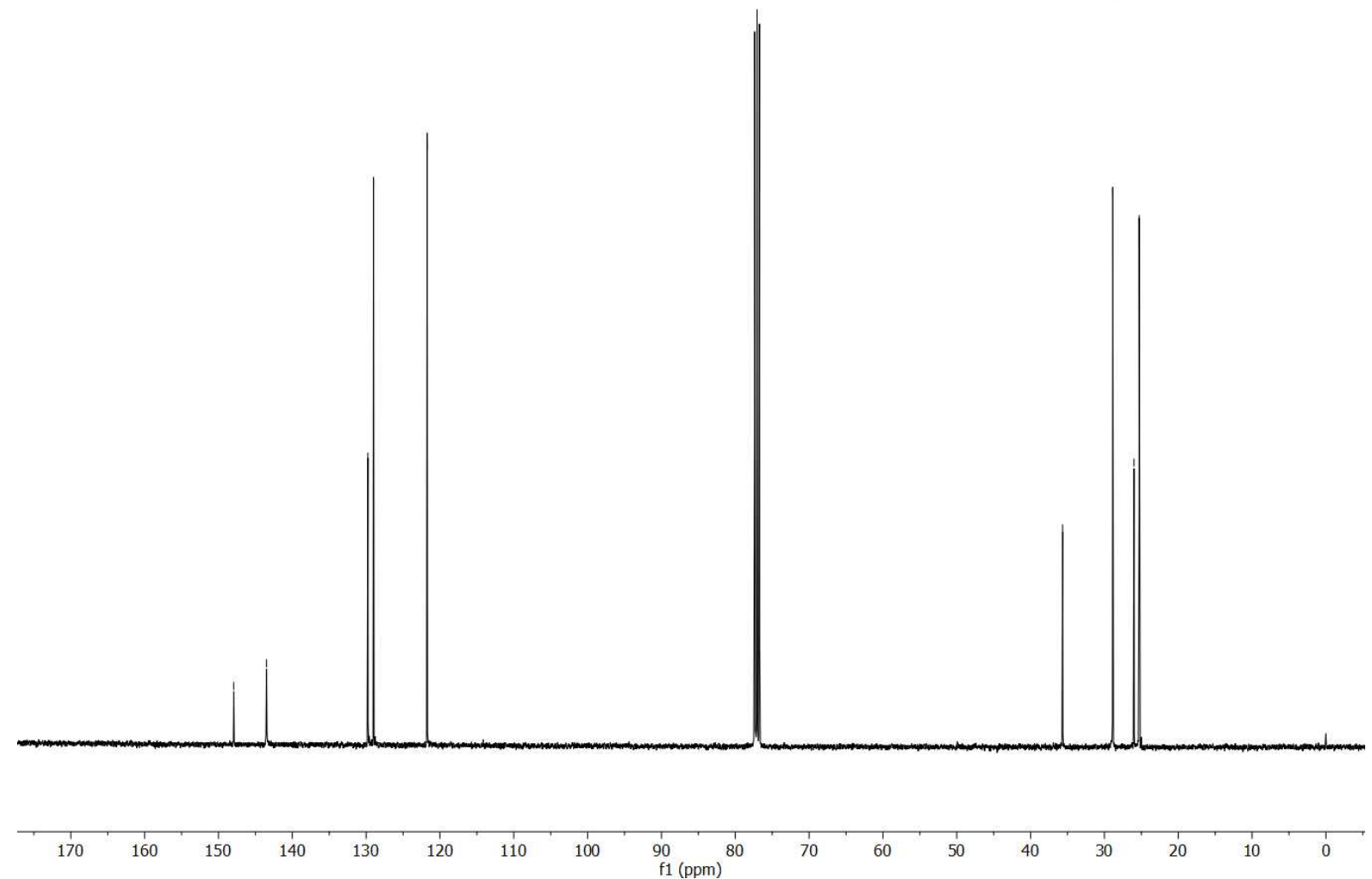

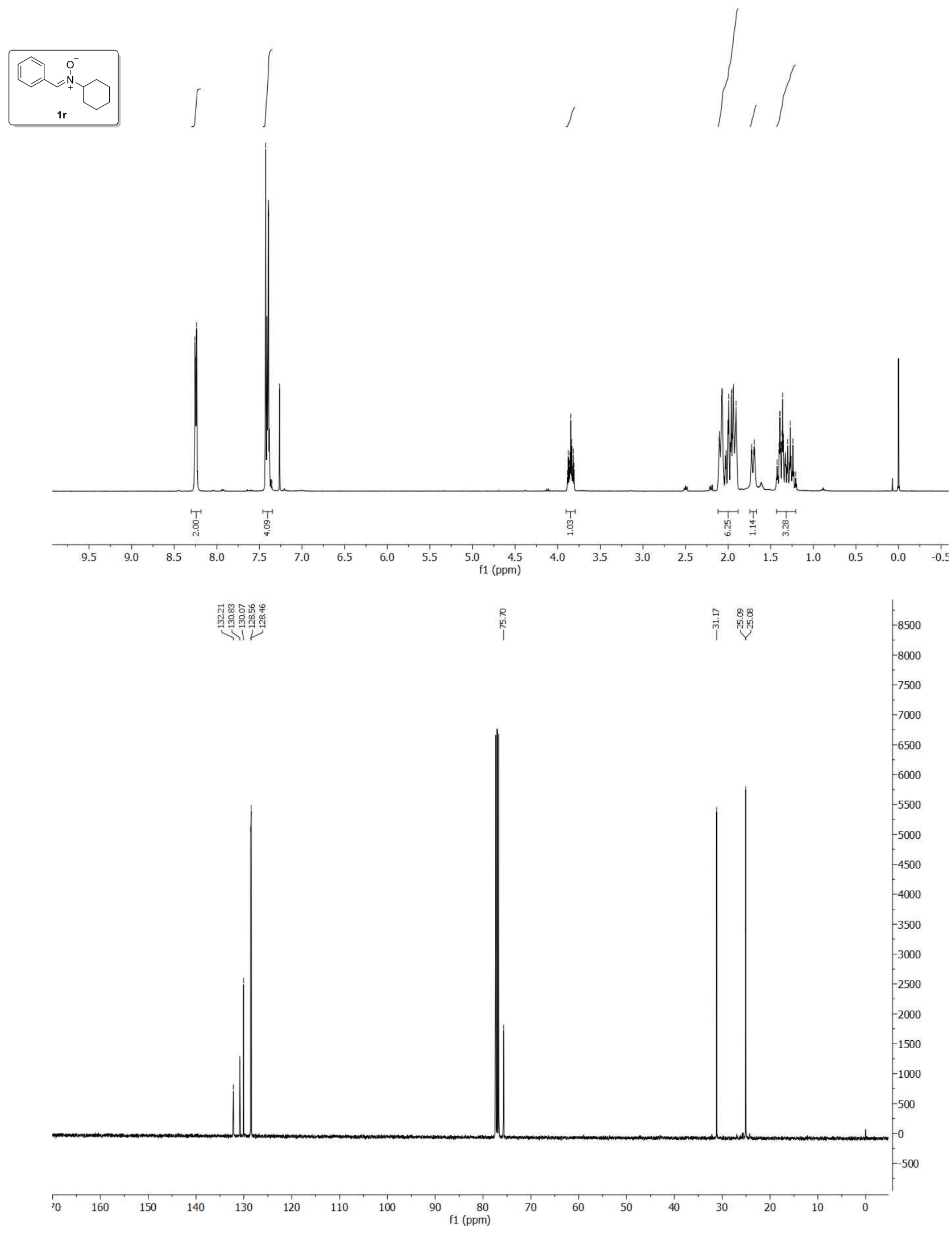

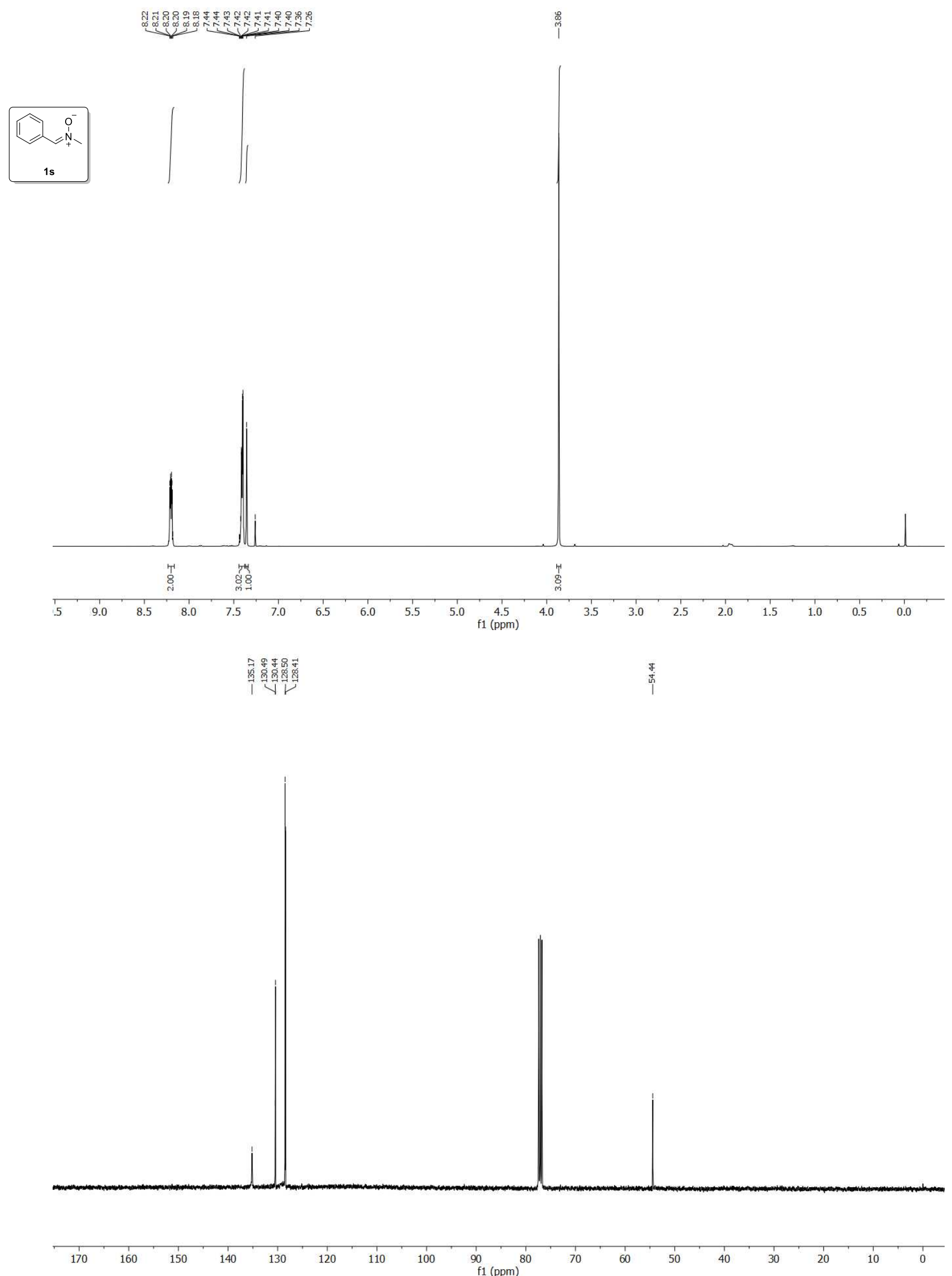

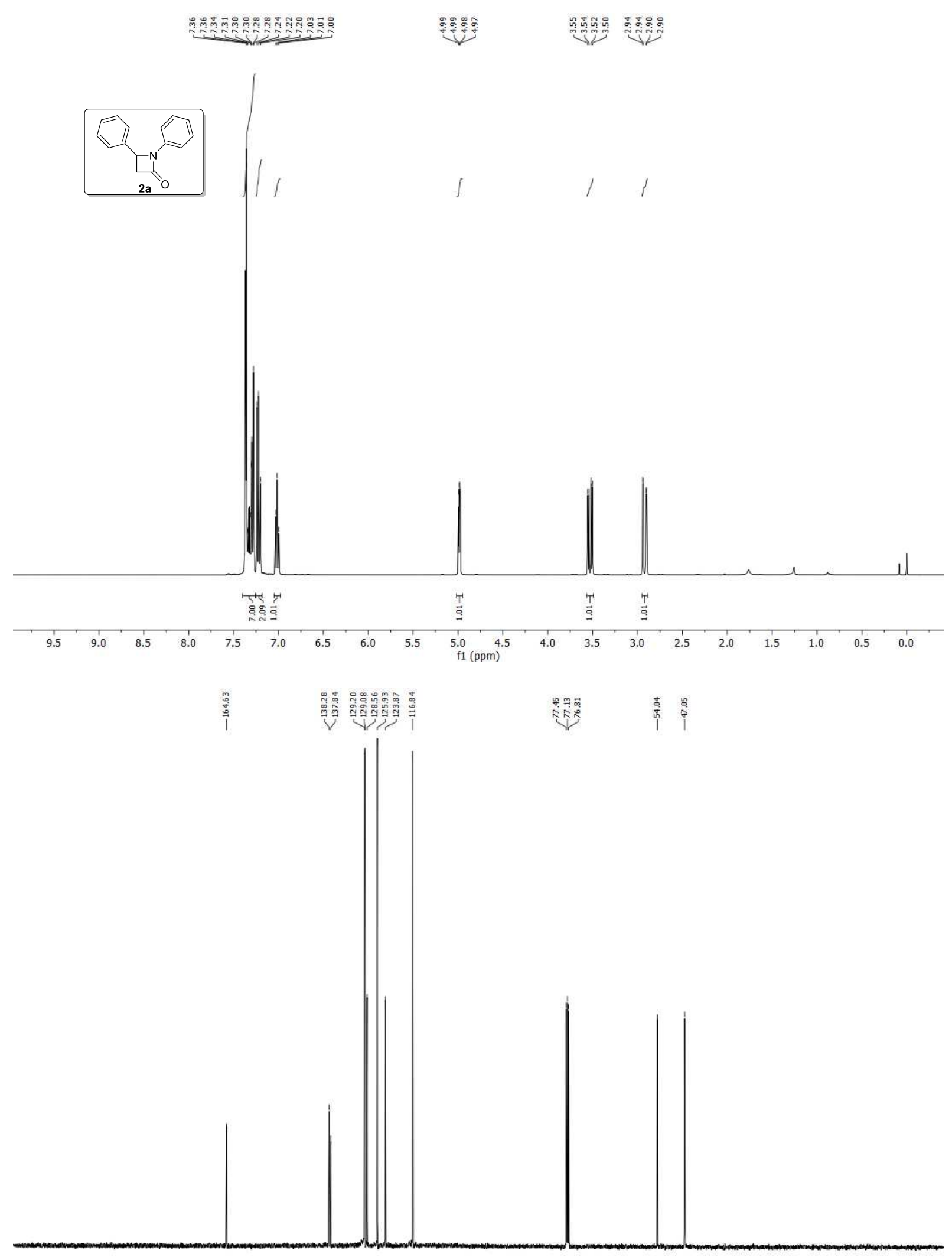

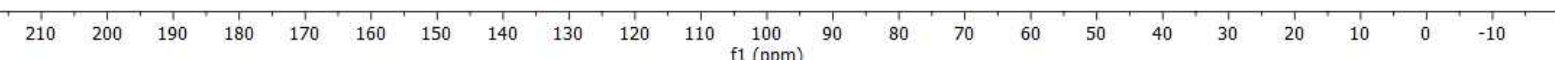



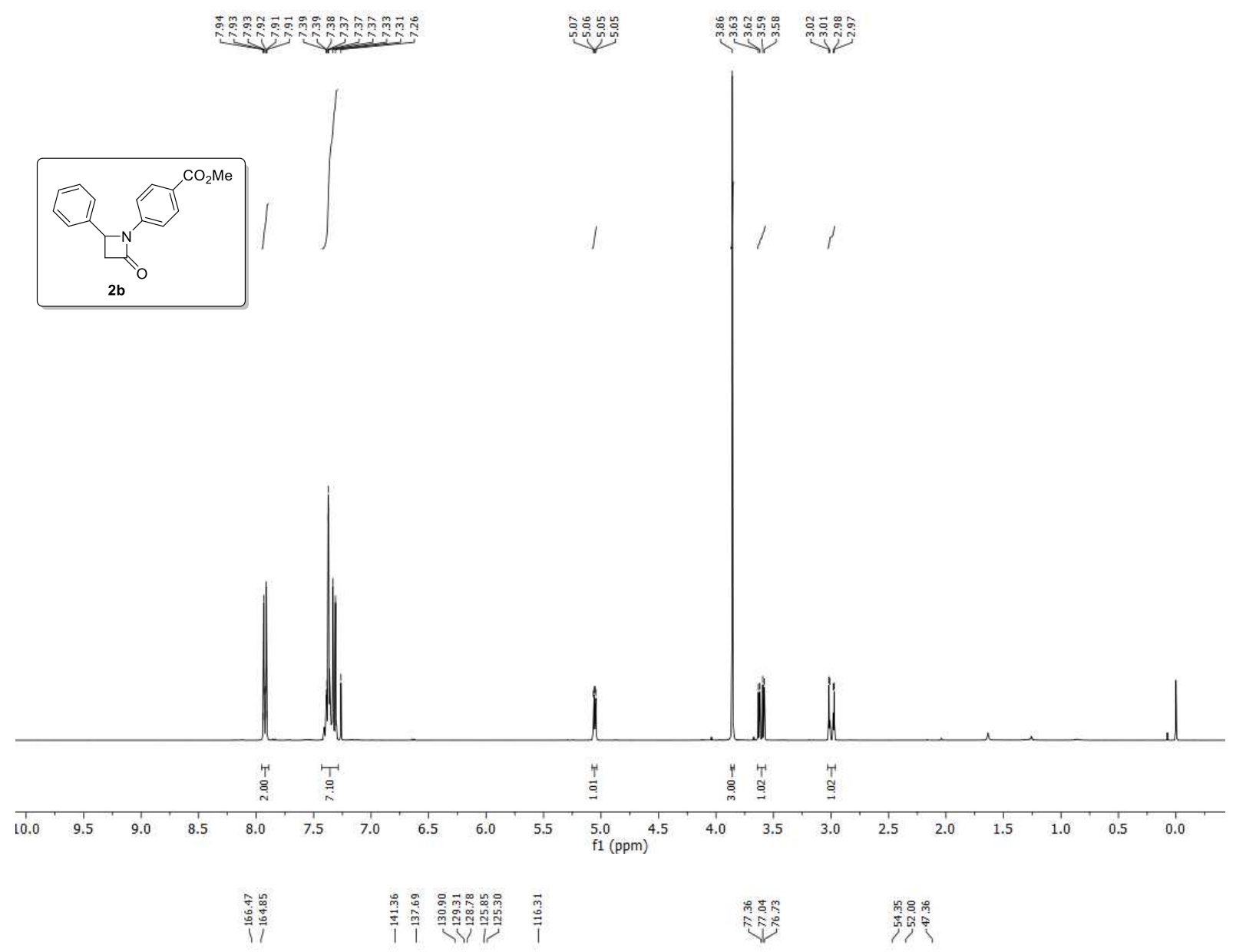

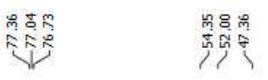
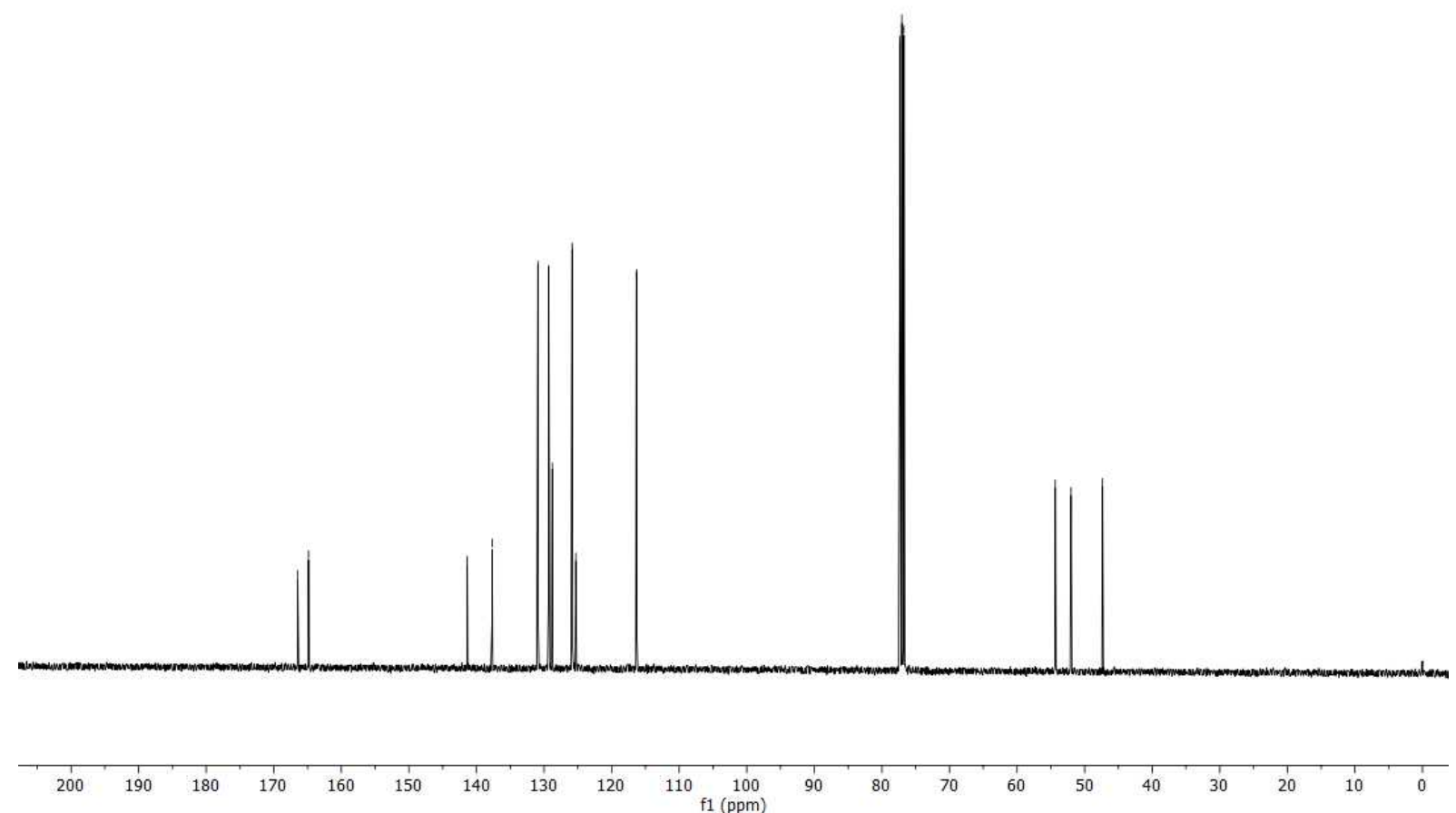


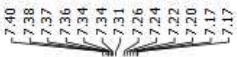

sog

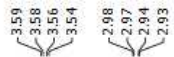

(1)
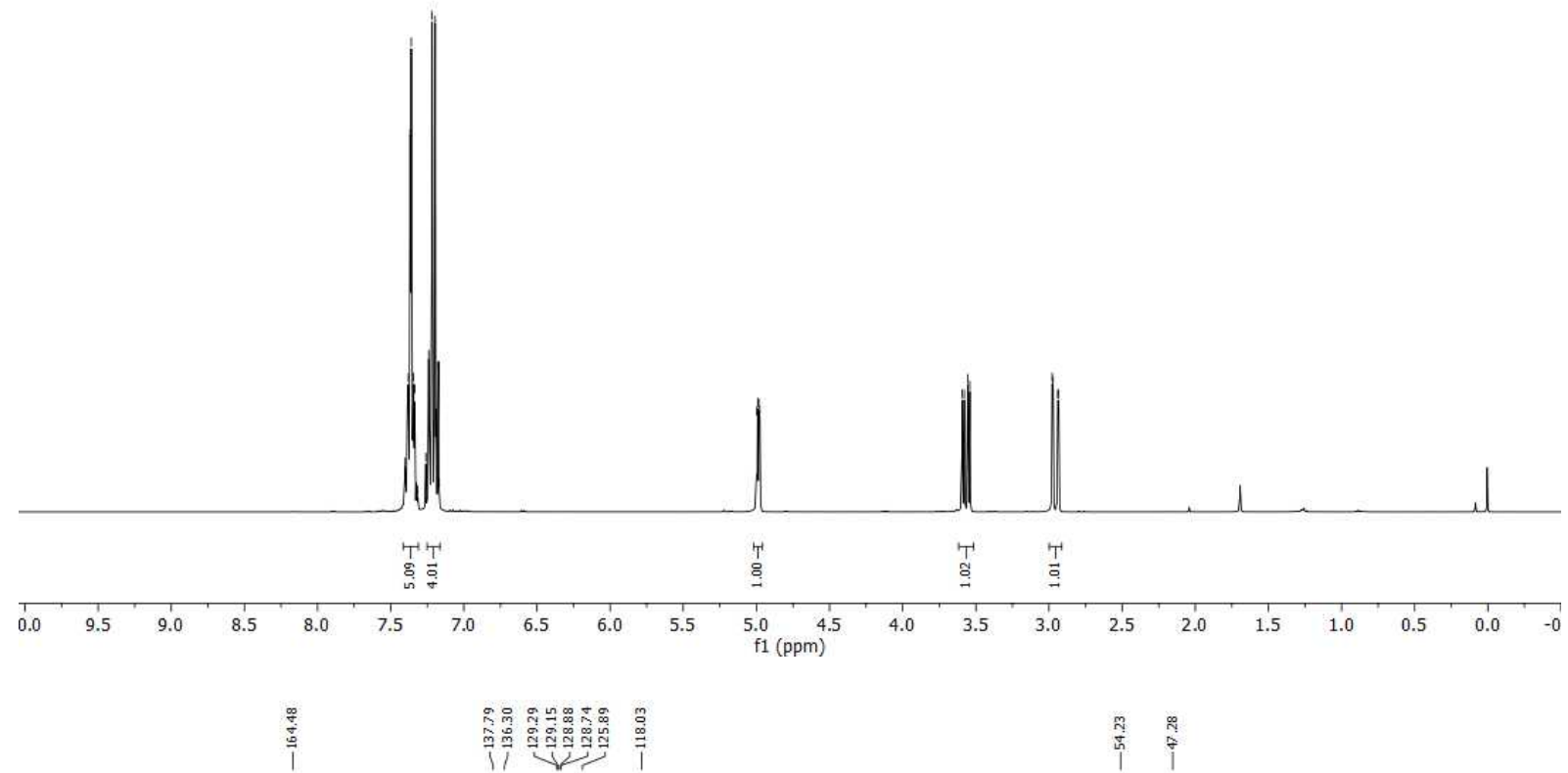

漓

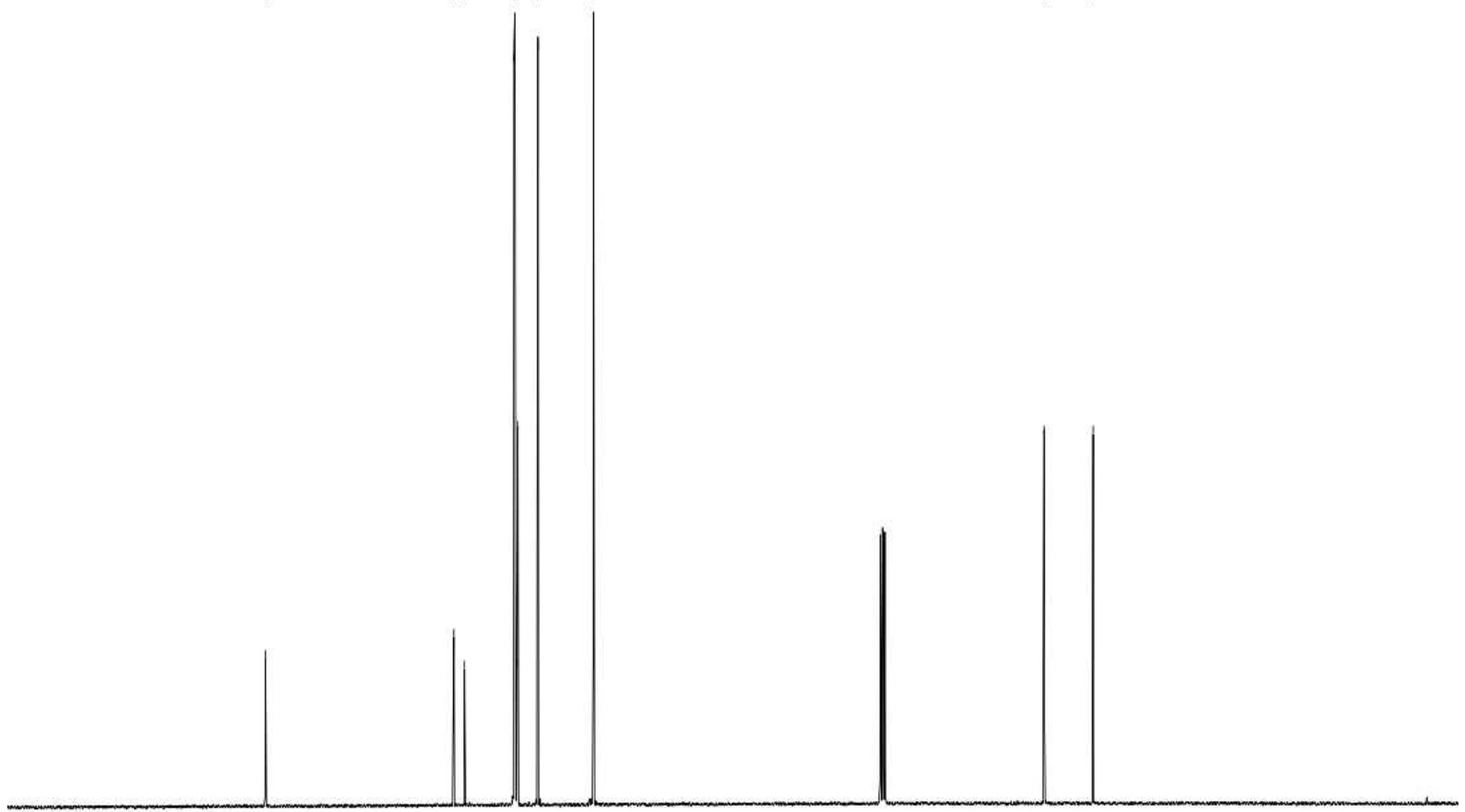

00
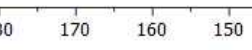

100 f1 

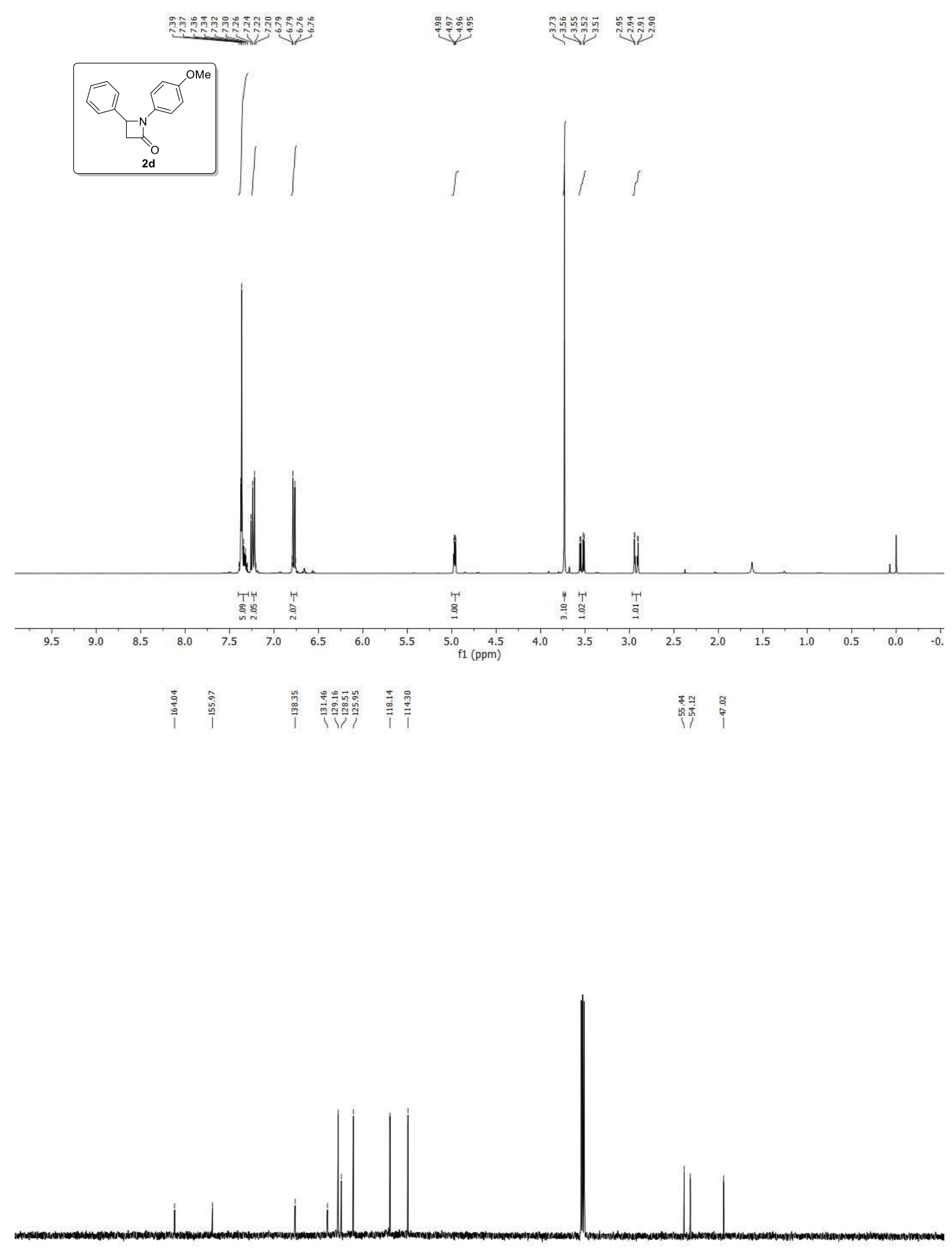

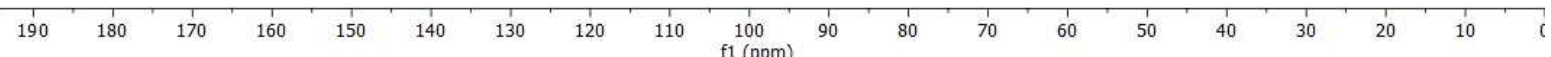



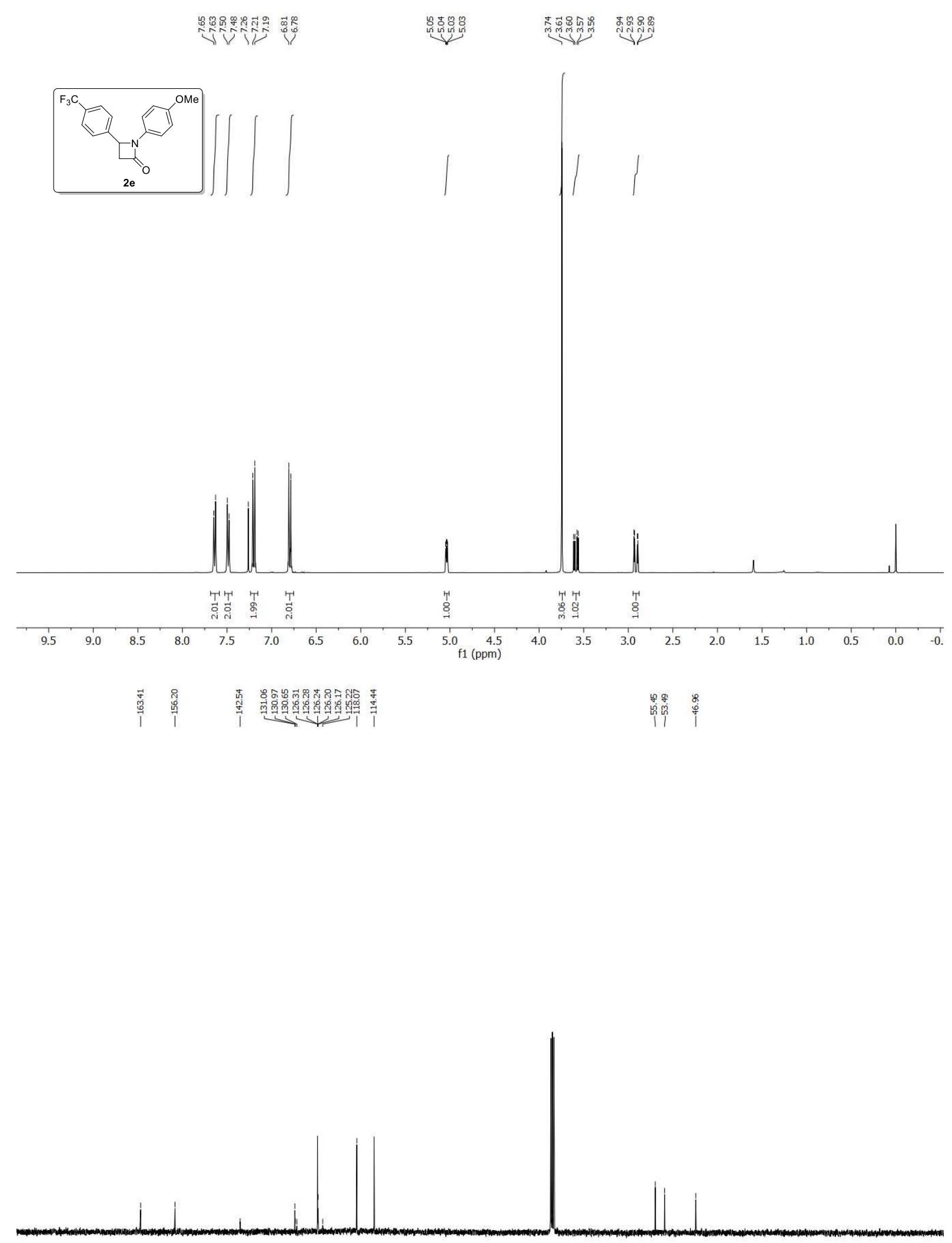

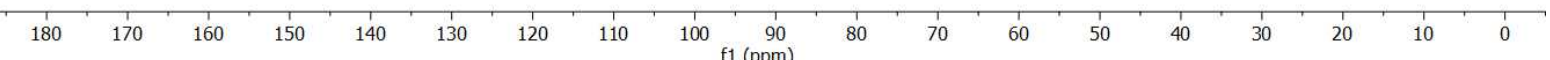




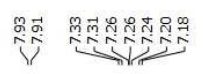

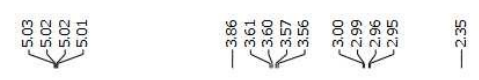
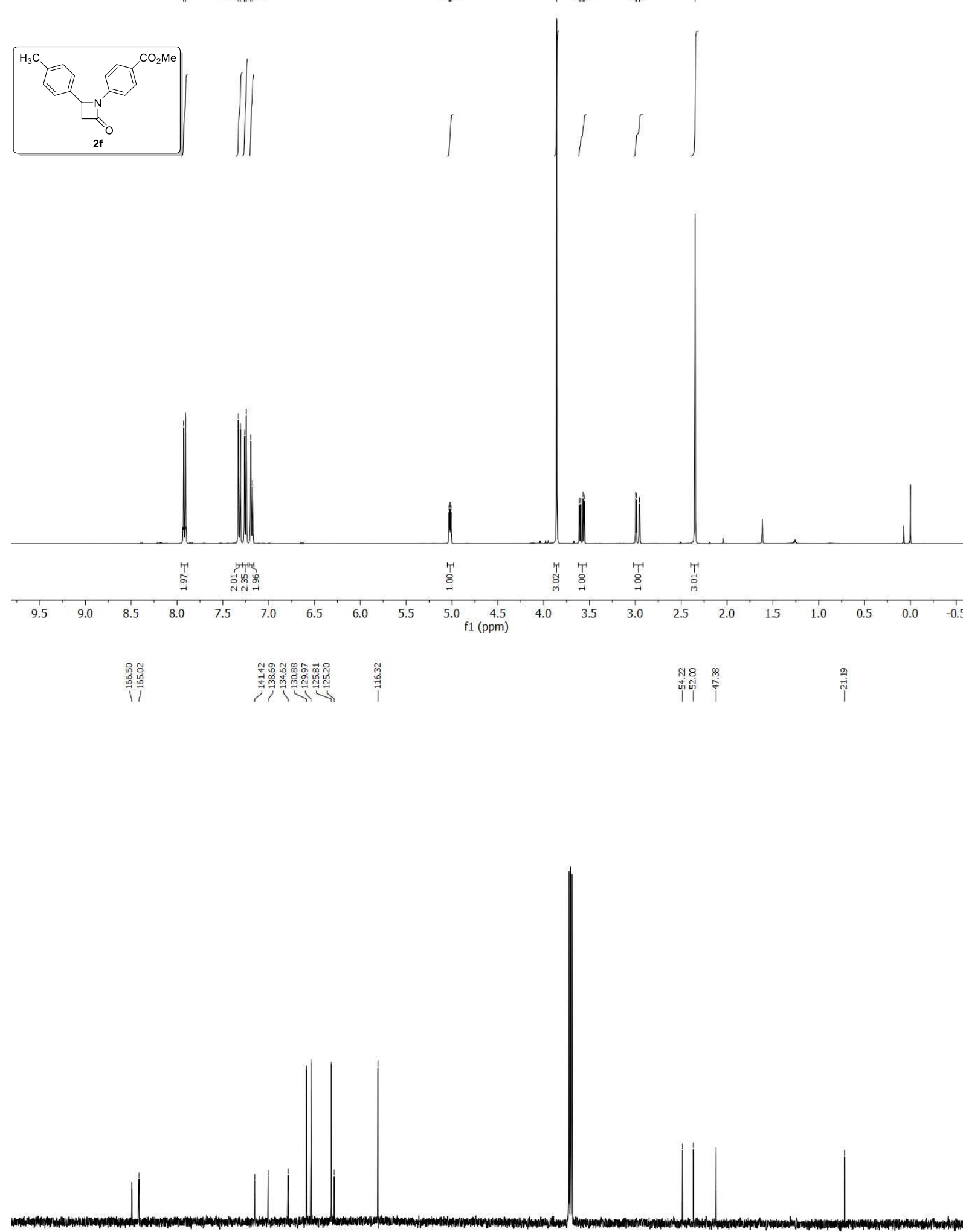

90
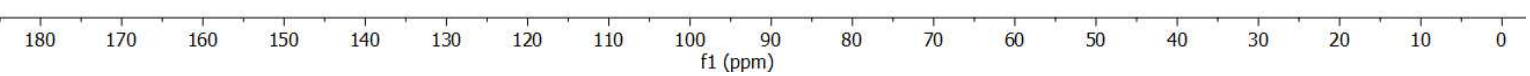


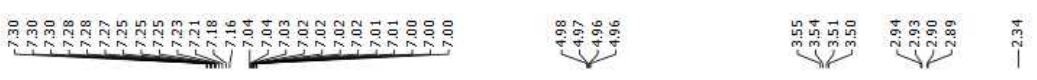
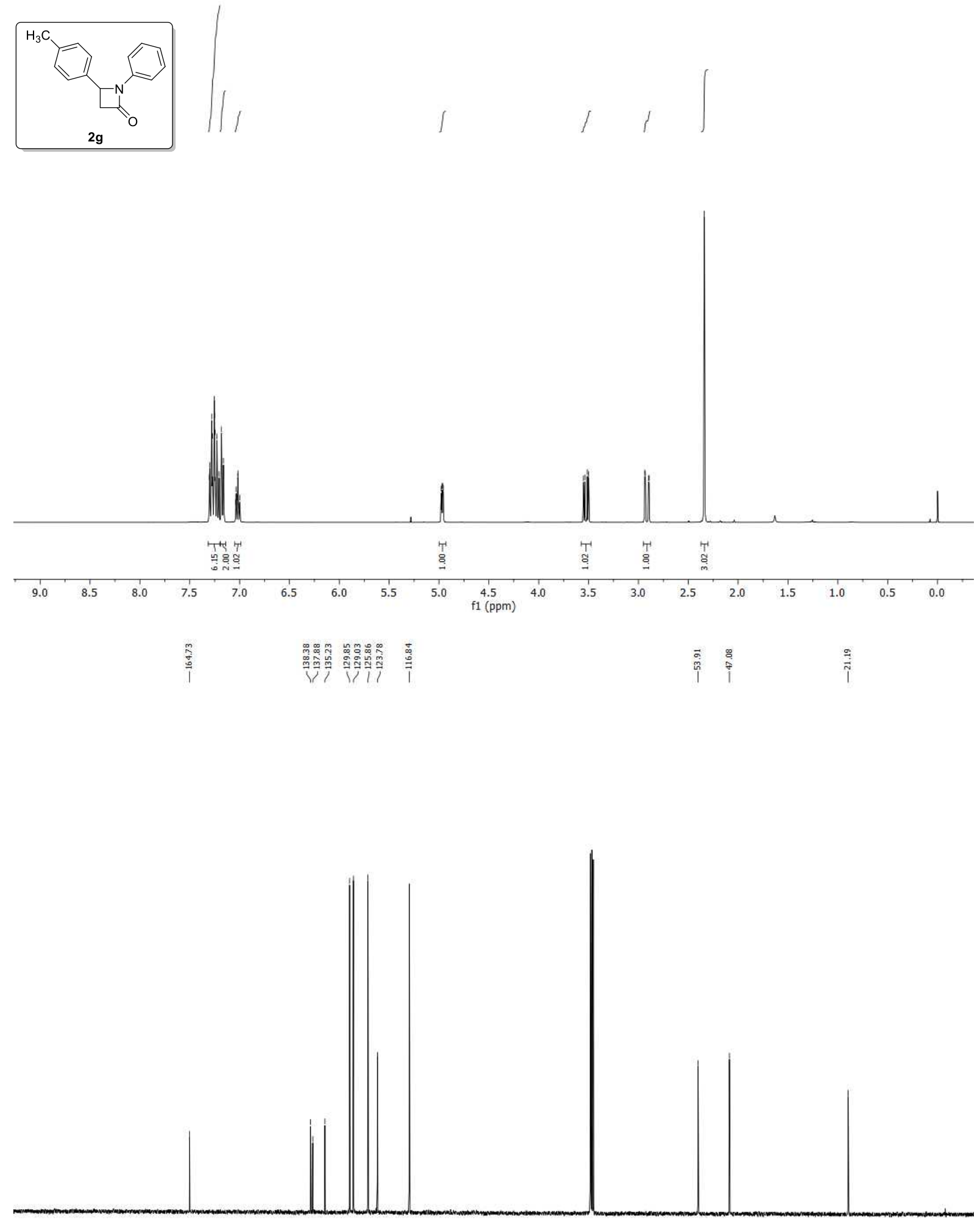

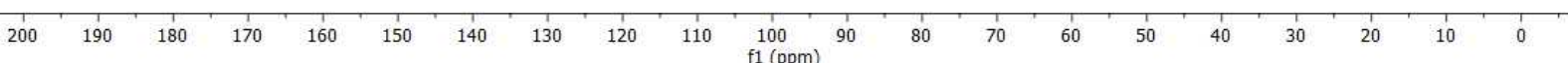




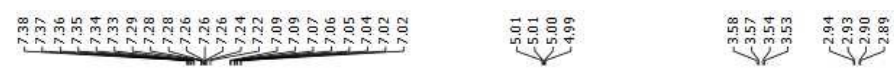
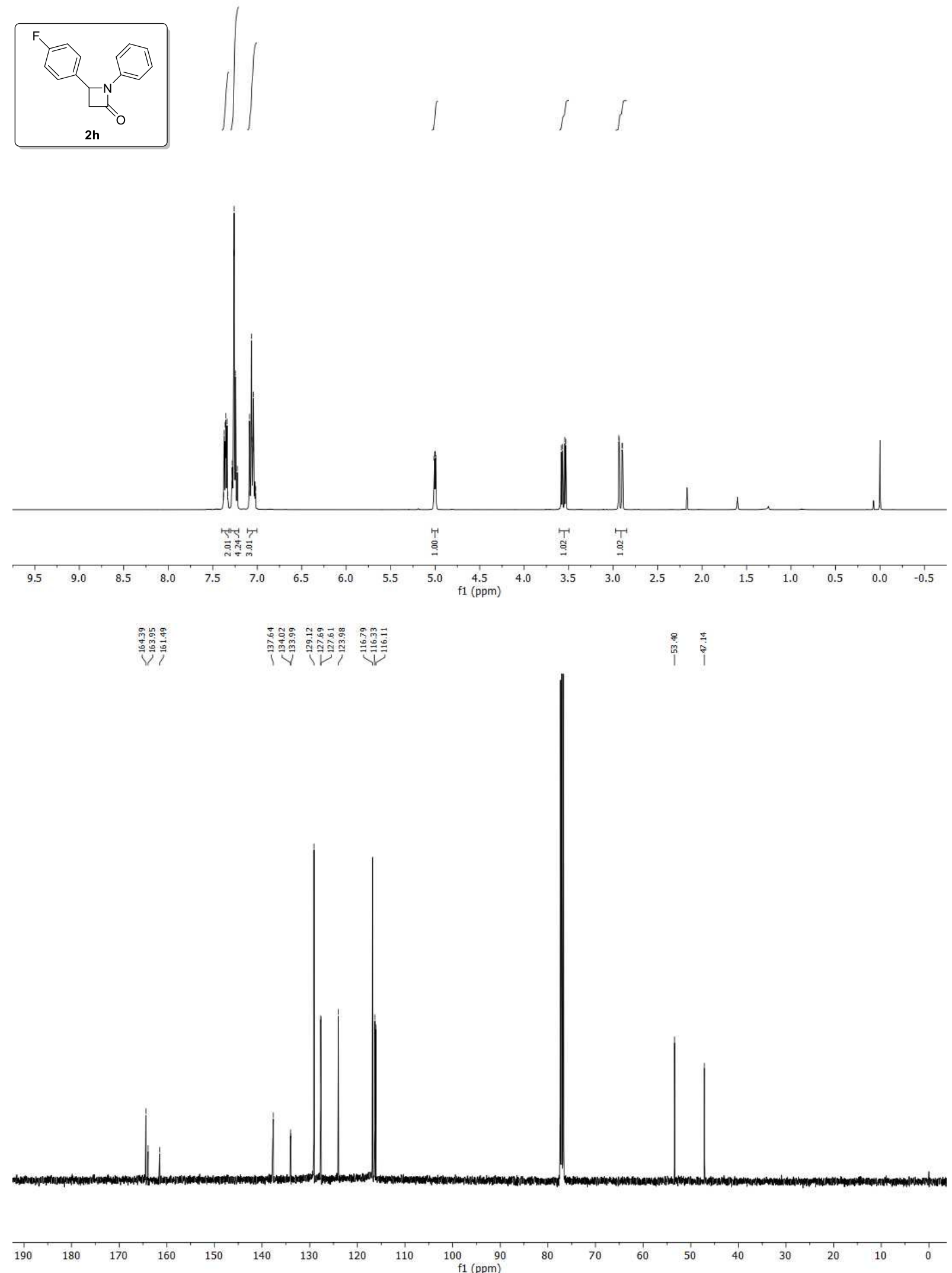

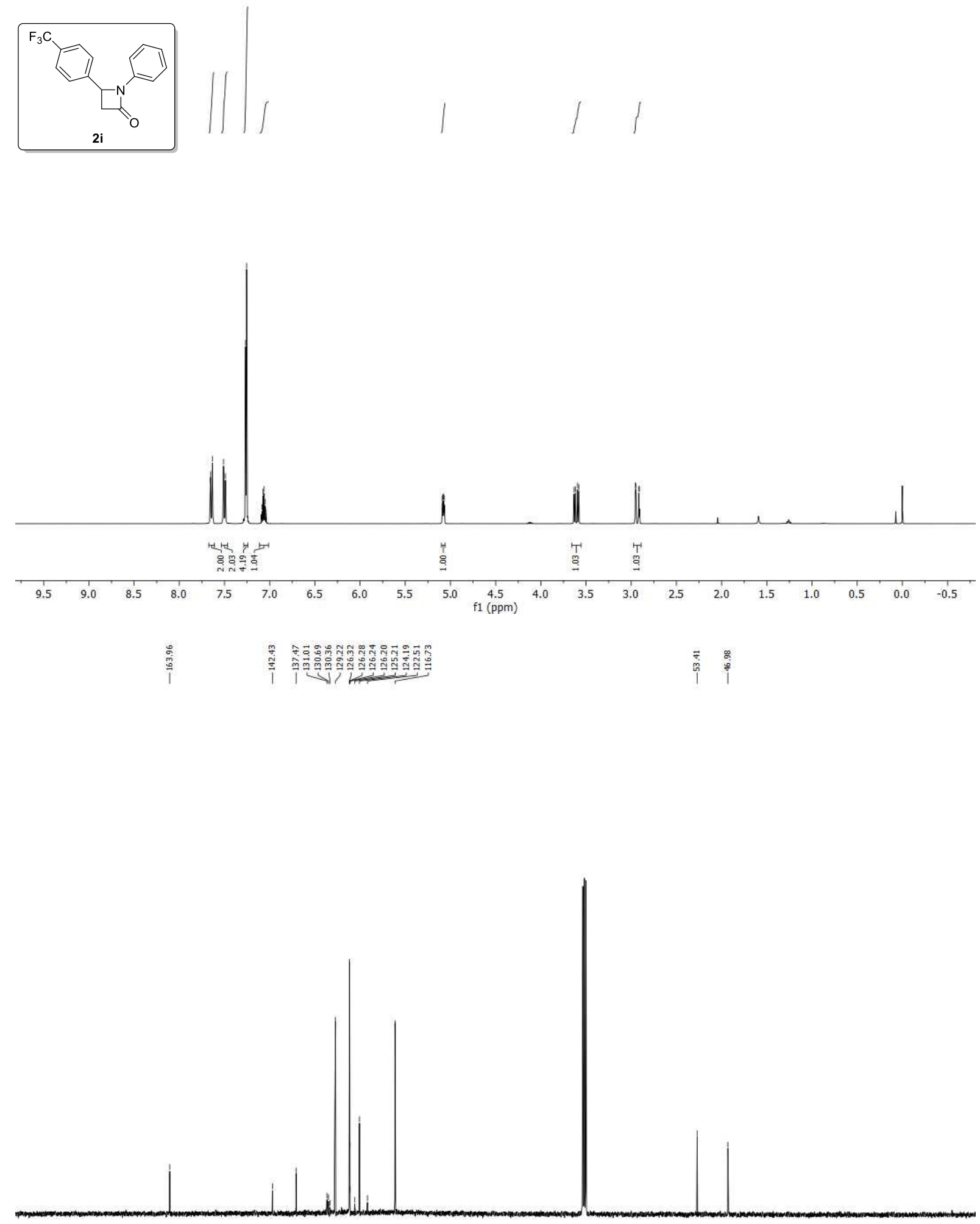

\begin{tabular}{llllllllll}
190 & 180 & 170 & 160 & 150 & 140 & 130 & 120 & 110 & 100 \\
\hline 100
\end{tabular}
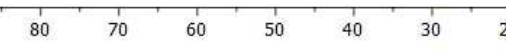

$1 0 \longdiv { 1 }, 0$ 


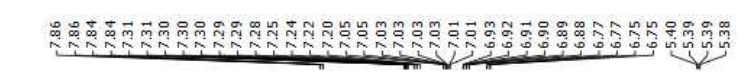

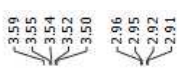
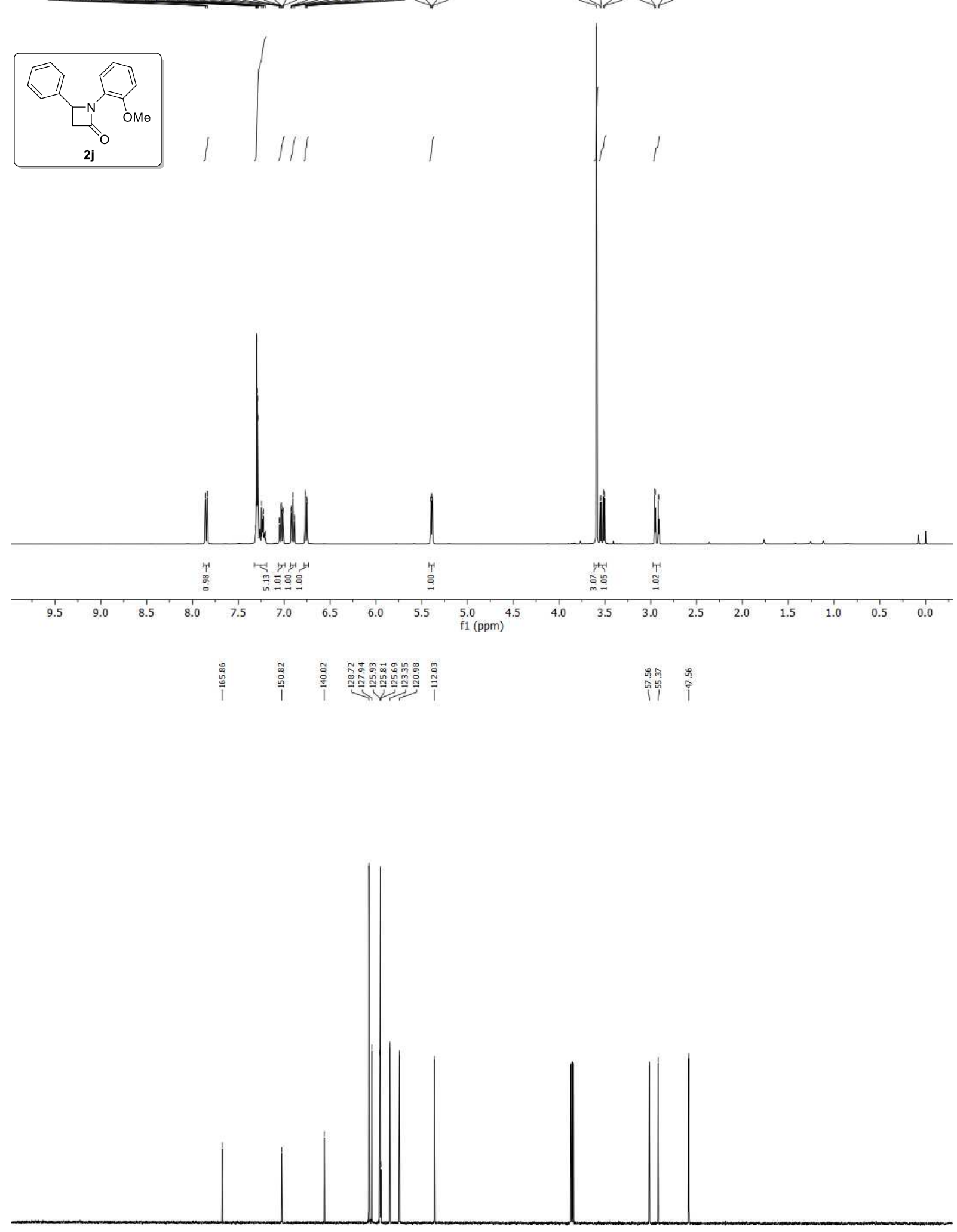

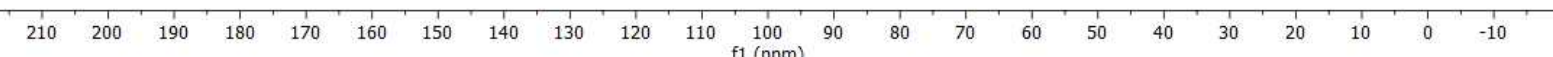



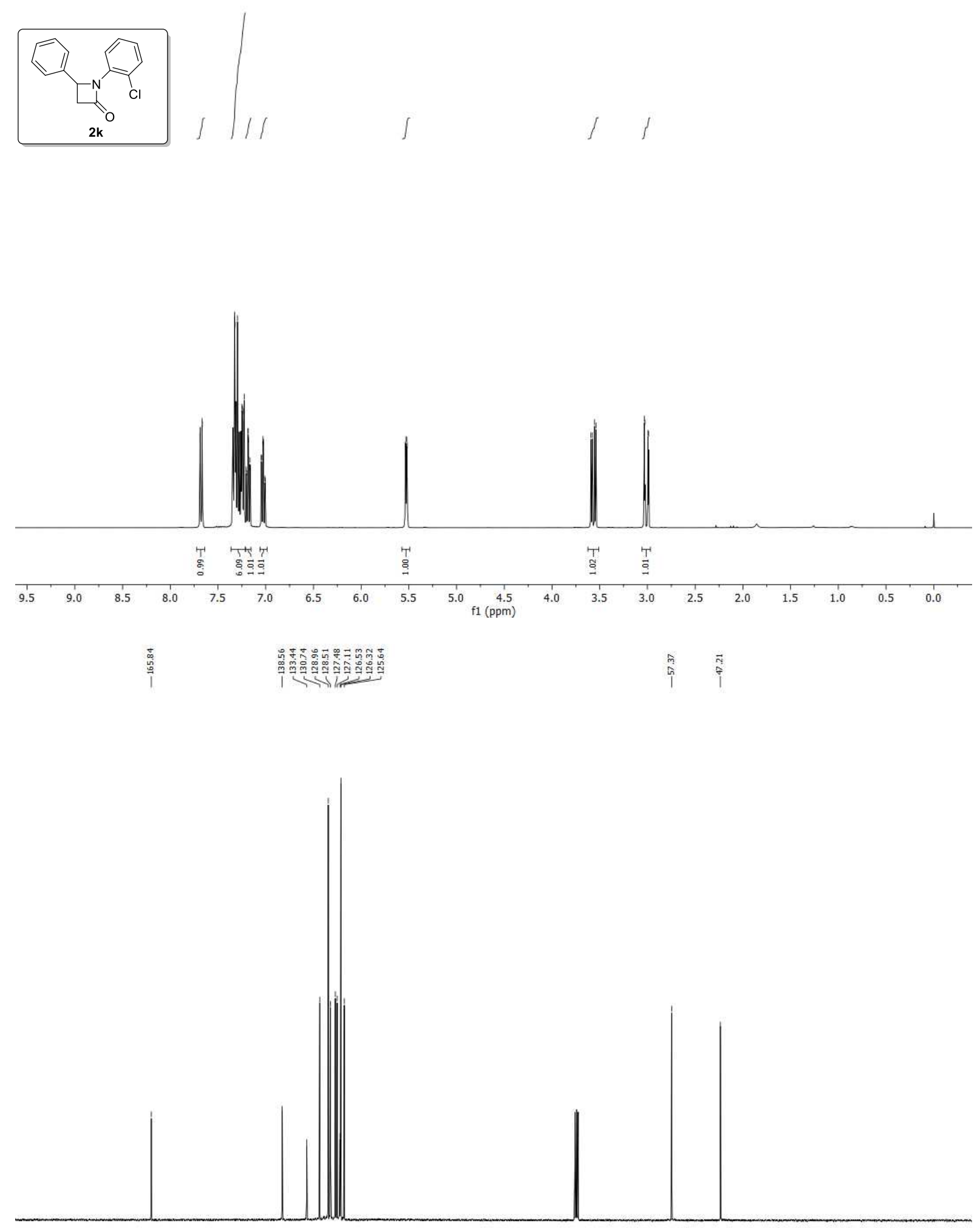

$\begin{array}{rrrrrrrrrr}190 & 180 & 170 & 160 & 150 & 140 & 130 & 120 & 110 & 100 \\ \mathrm{f} 1(\mathrm{ppm})\end{array}$ 


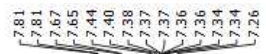

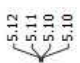

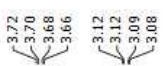

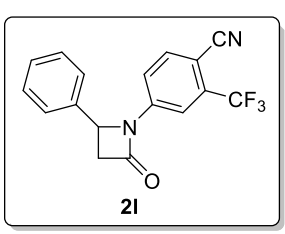

11

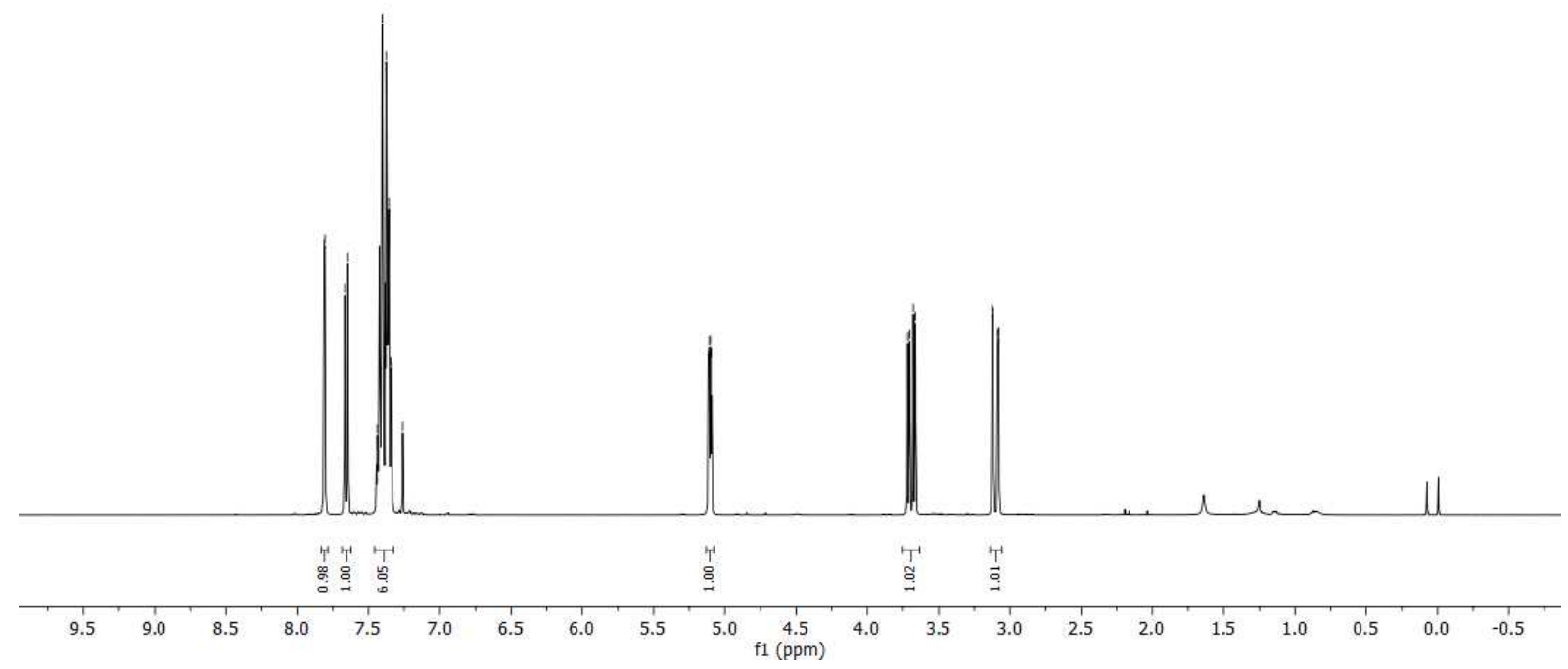

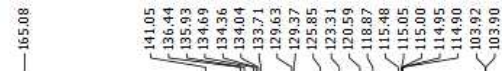

草

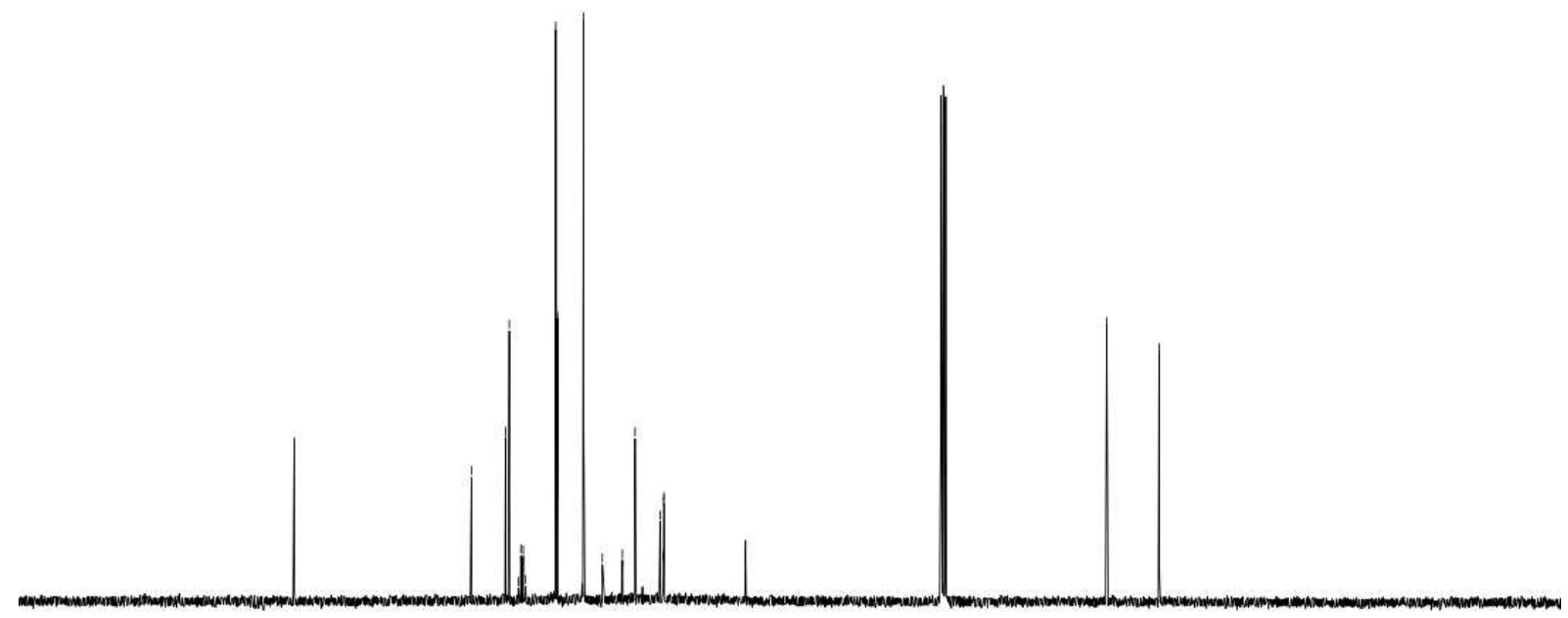

200

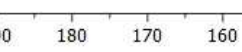

$\begin{array}{lll}1 & 150 & 140\end{array}$

$130 \quad 12$

100
$\mathrm{f} 1(\mathrm{ppm})$ 

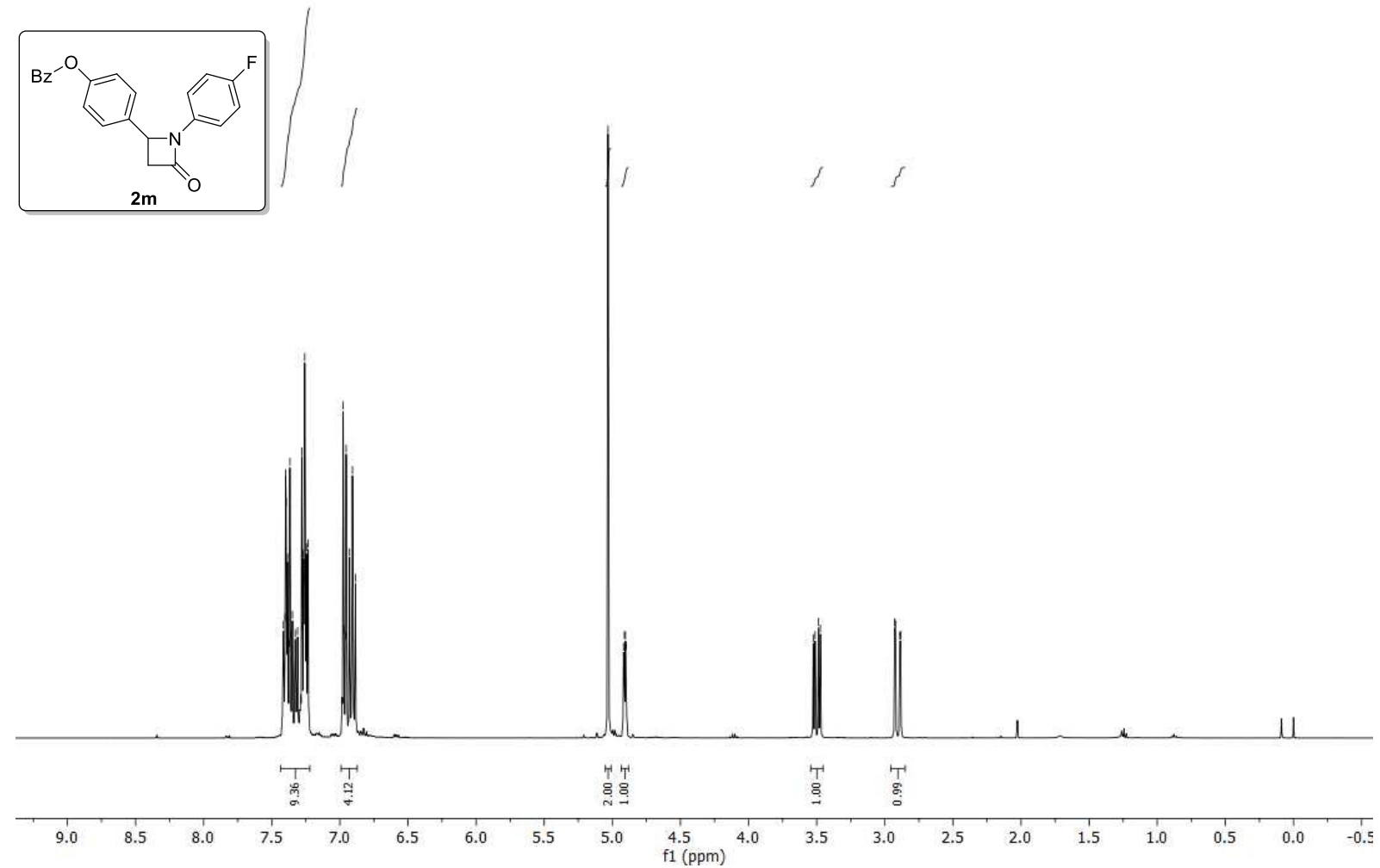

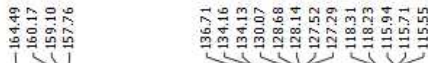

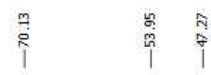

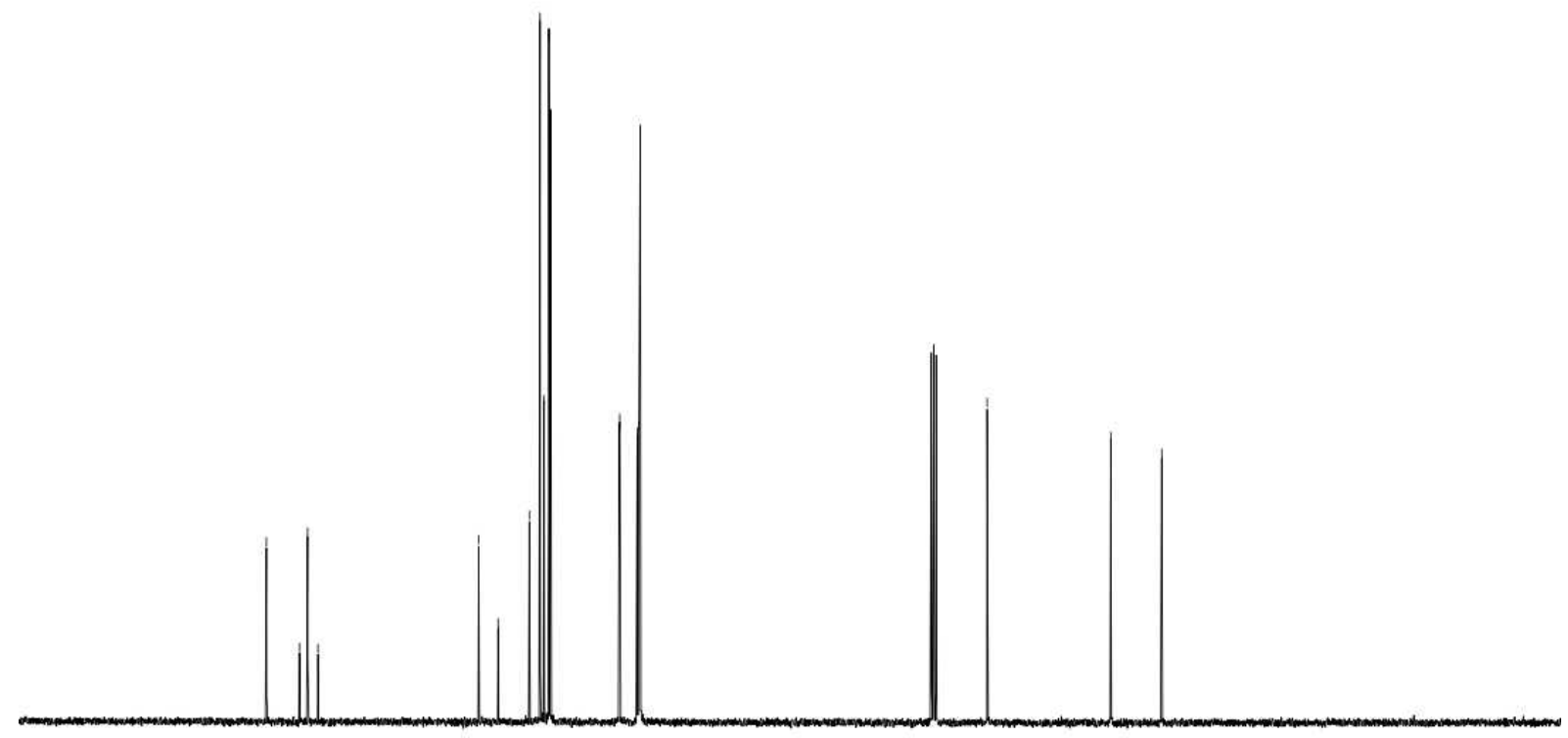

$\begin{array}{llllllllll}190 & 180 & 170 & 160 & 150 & 140 & 130 & 120 & 110 & 100 \\ 90\end{array}$
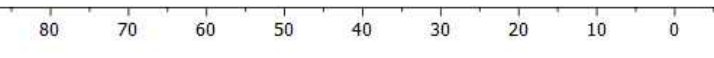


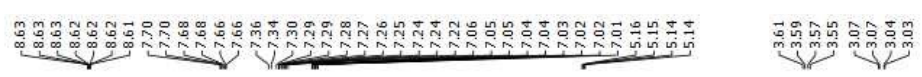
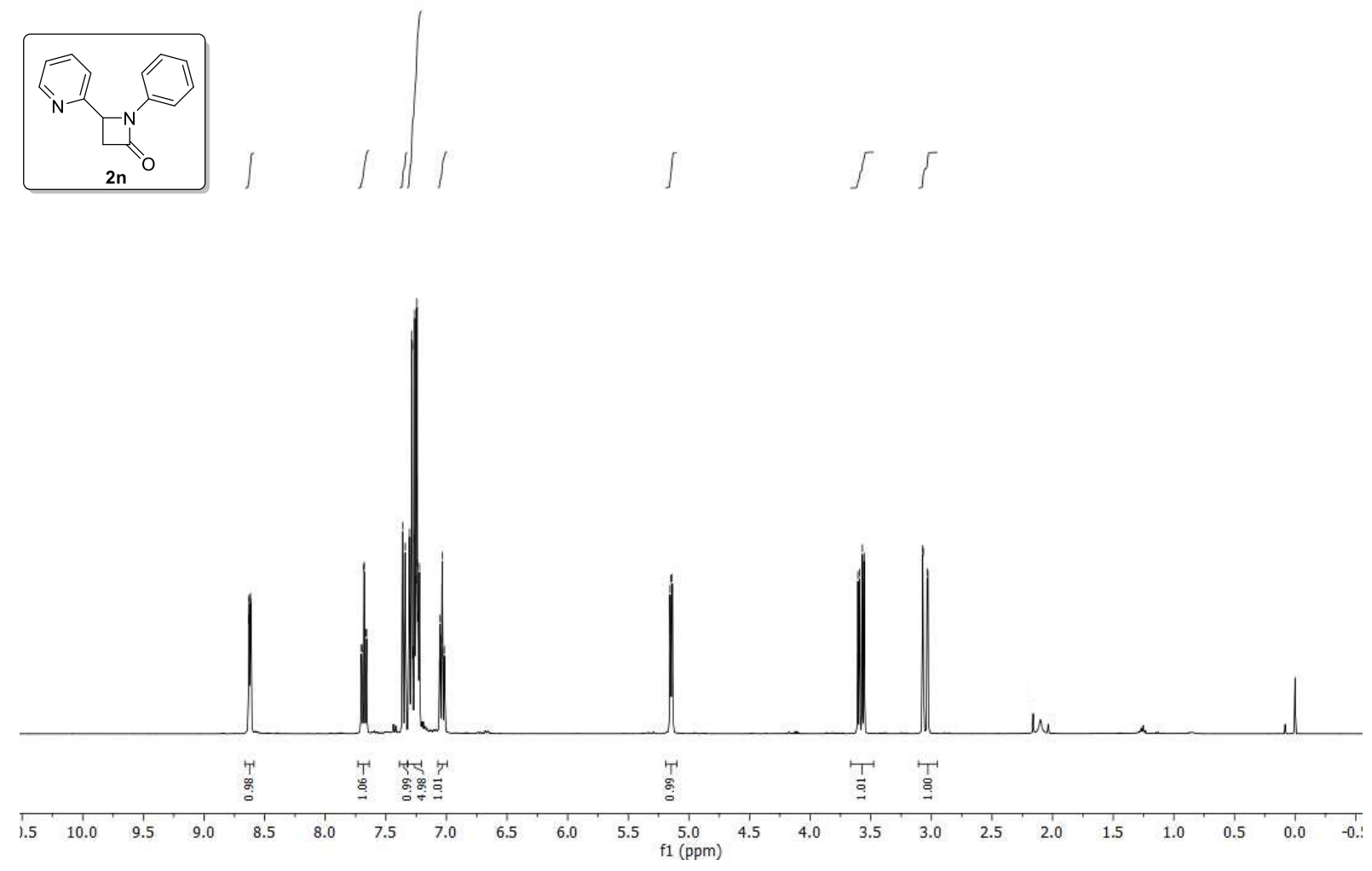

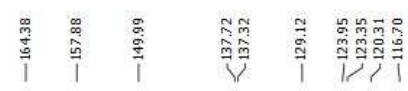

离证
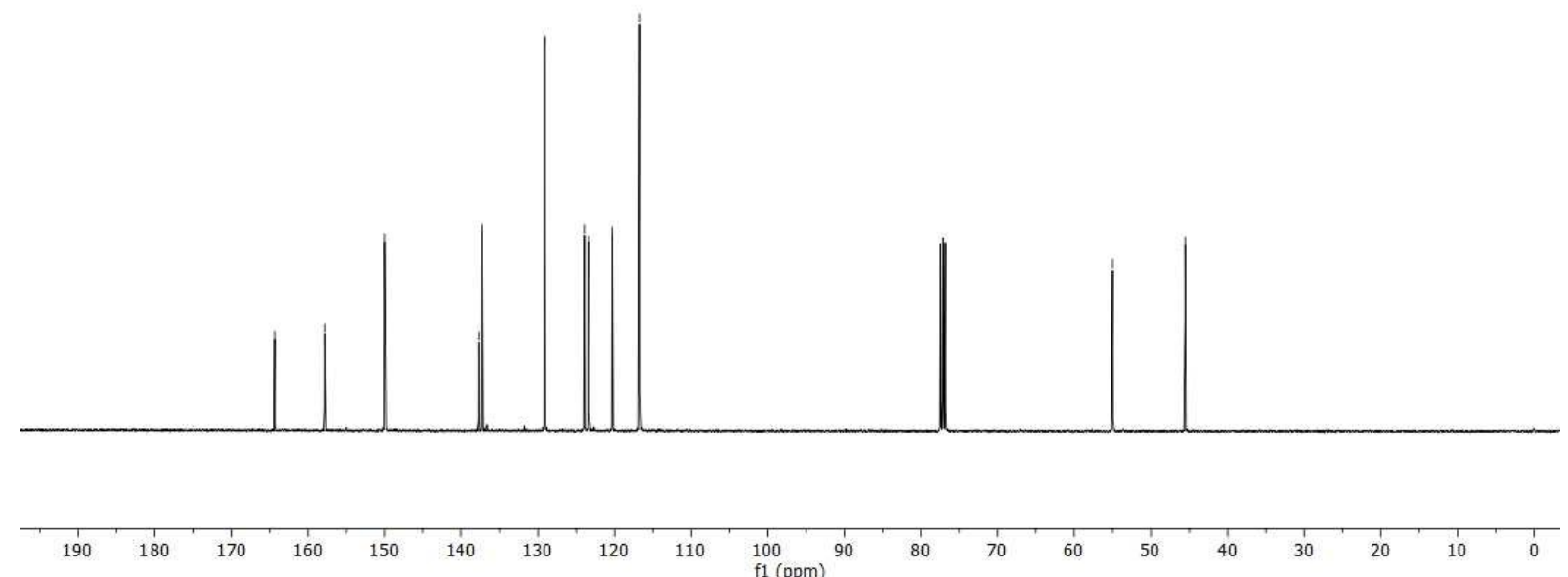

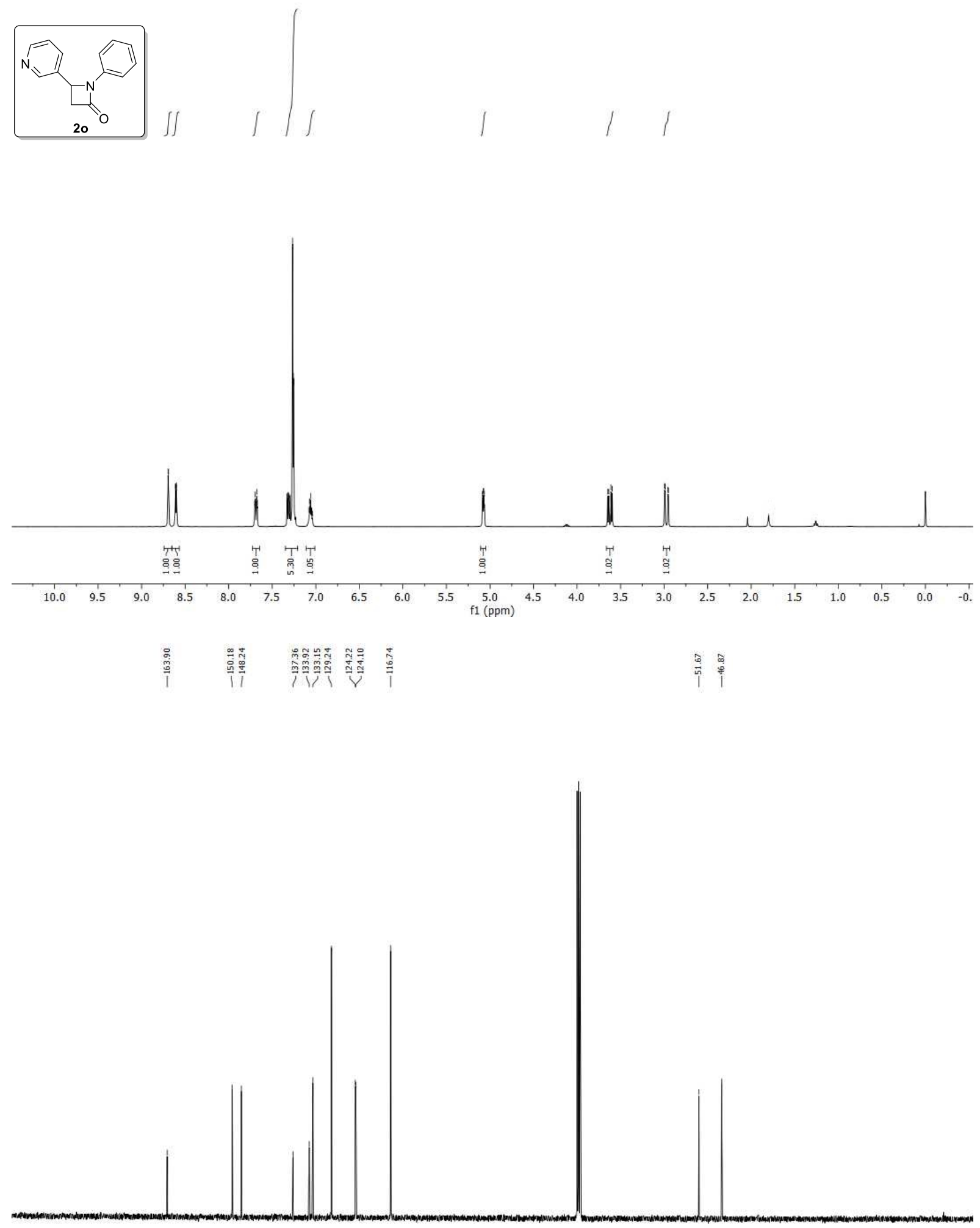

$\begin{array}{llllllllll}190 & 180 & 170 & 160 & 150 & 140 & 130 & 120 & 110 & 100 \\ 90\end{array}$ 


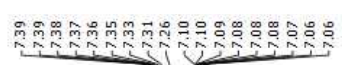

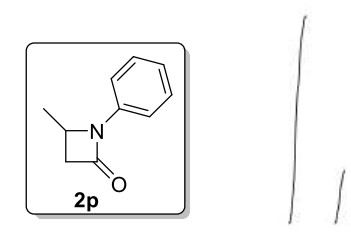

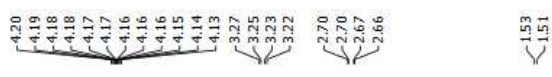

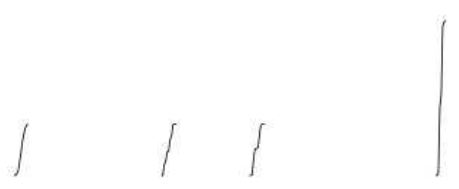

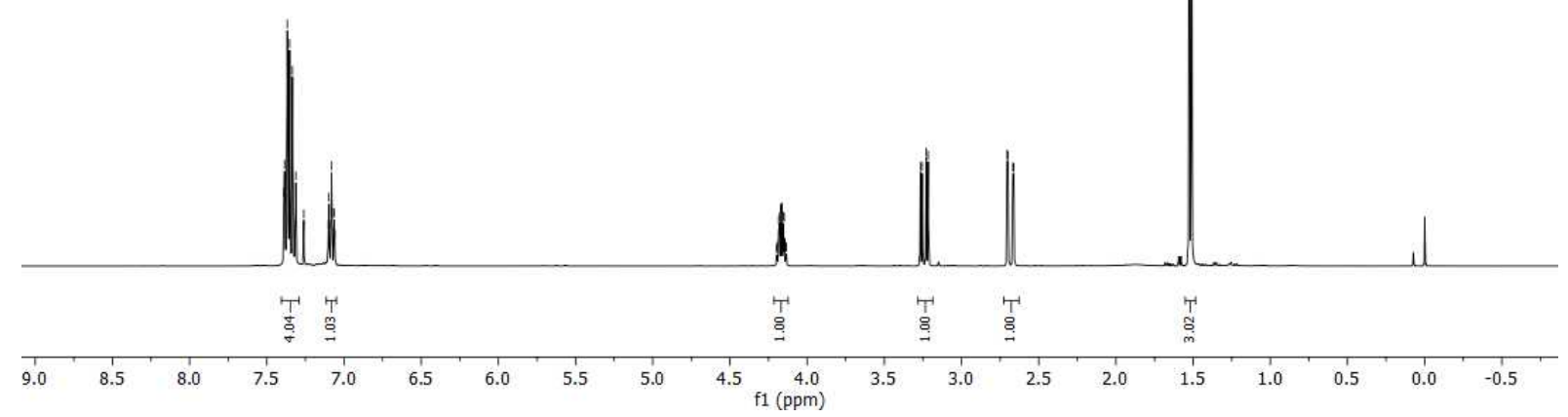

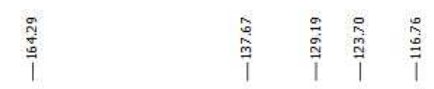

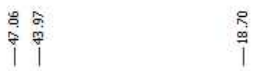

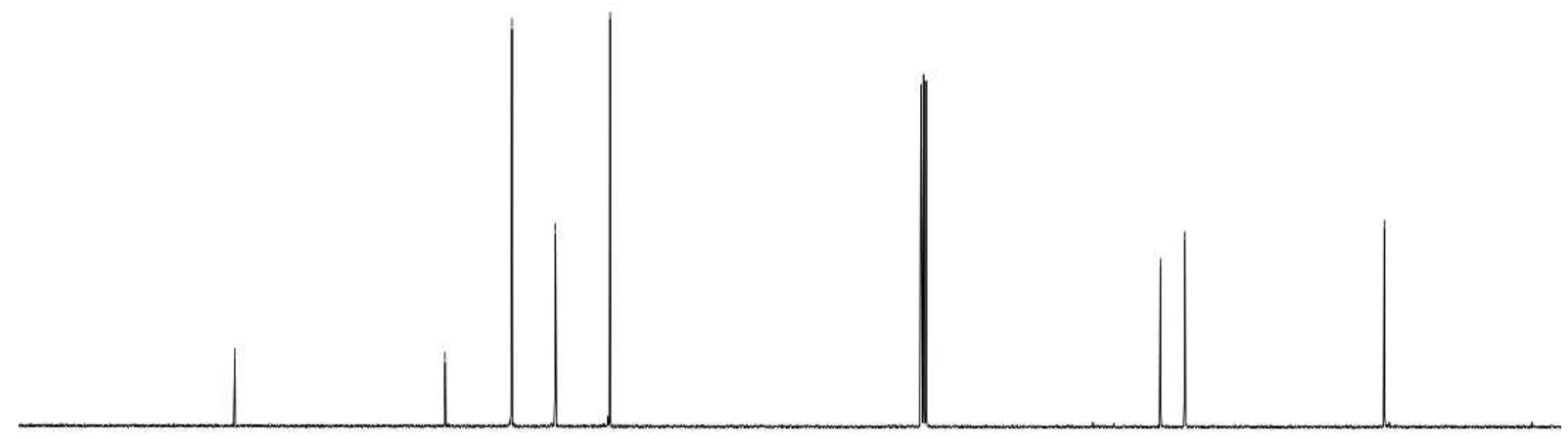

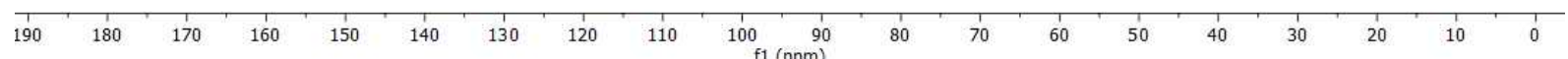




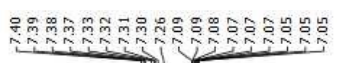

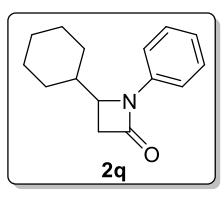

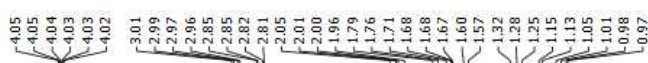

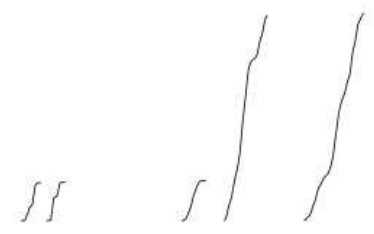

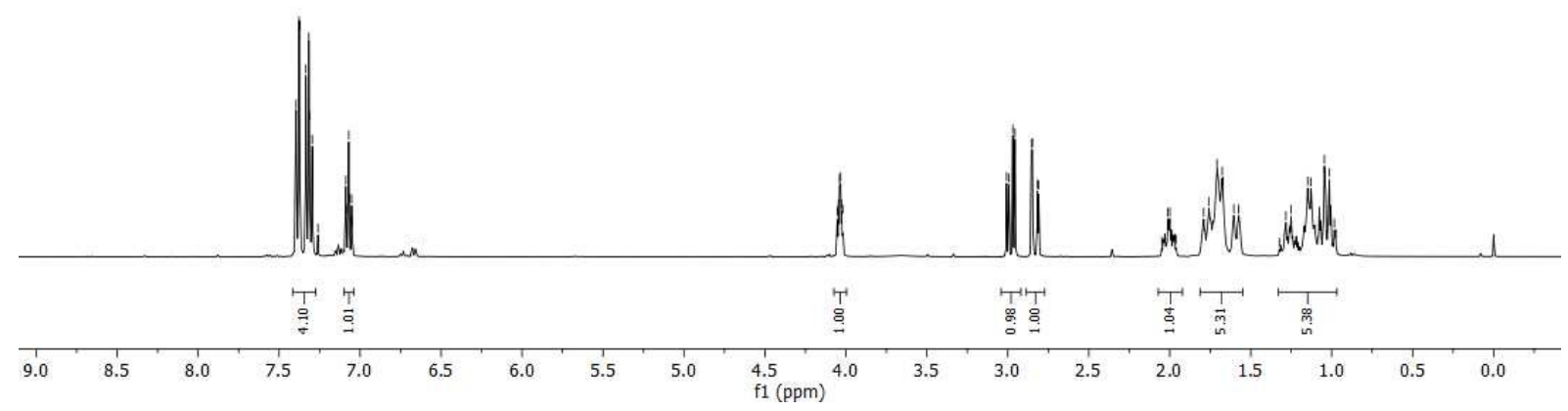

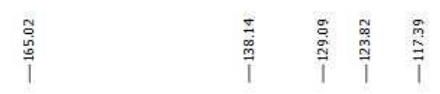

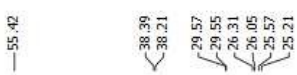

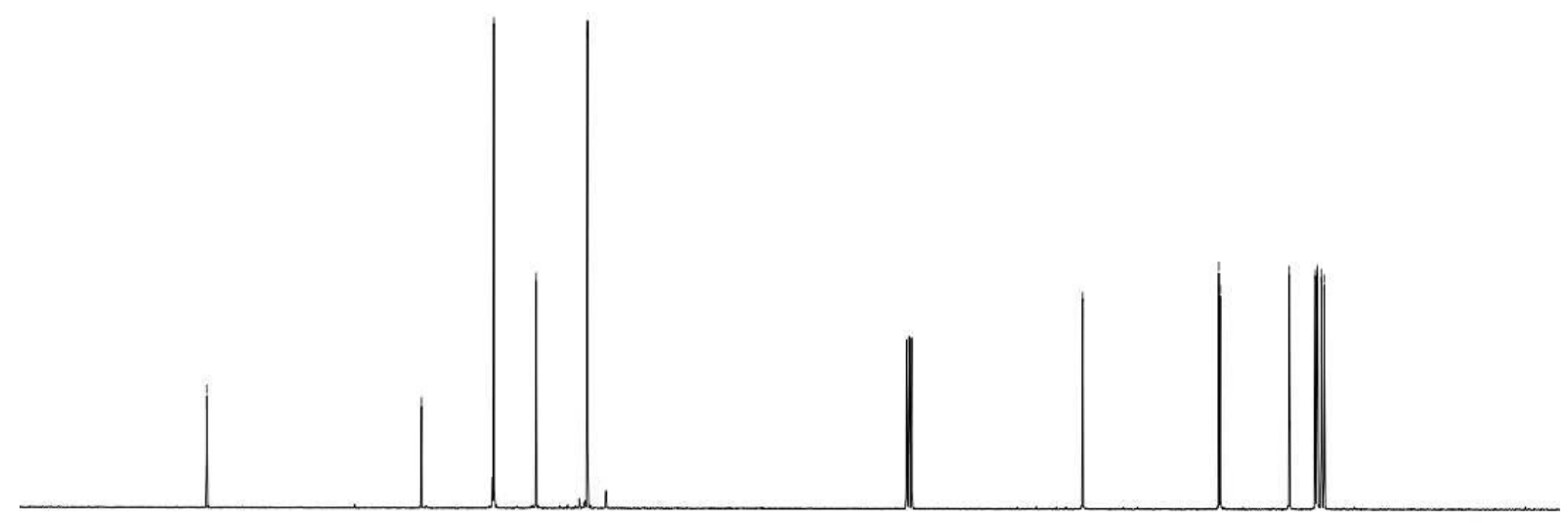

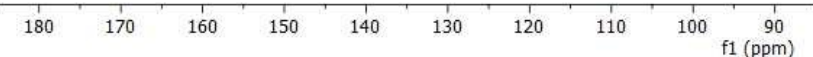




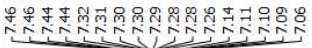

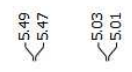

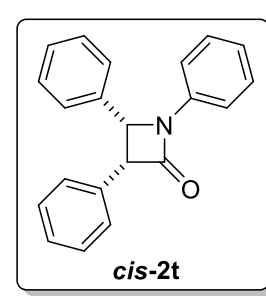

$1 / 1$

4.5
f1 $($ ppm)

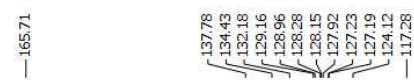

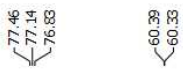

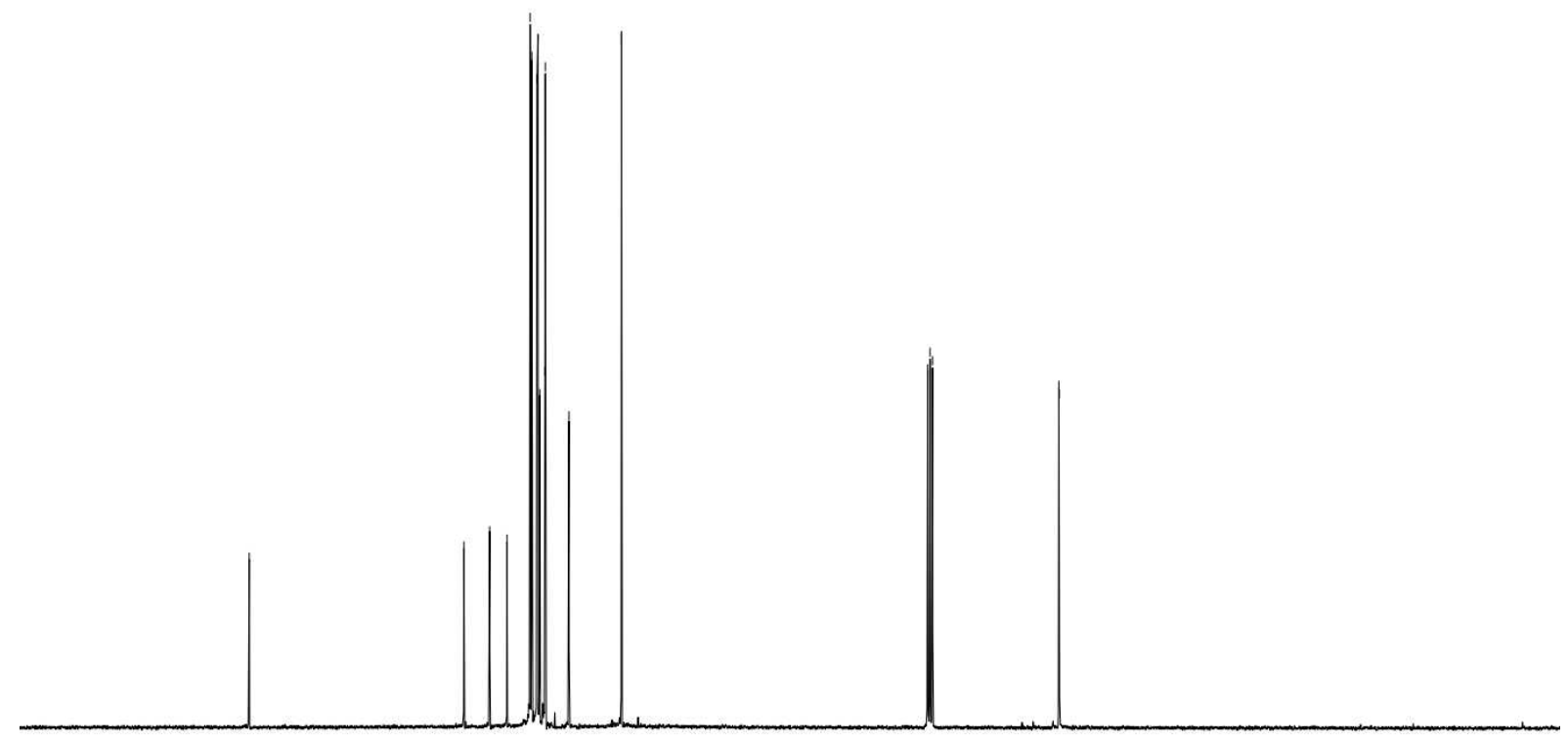

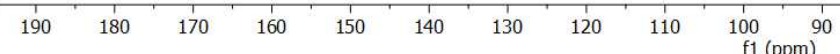

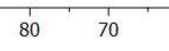

50

$\begin{array}{lllll}1 & 1 & 1 & 1 \\ 30 & 20 & 10 & 0\end{array}$ 


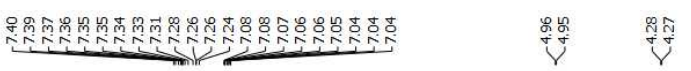
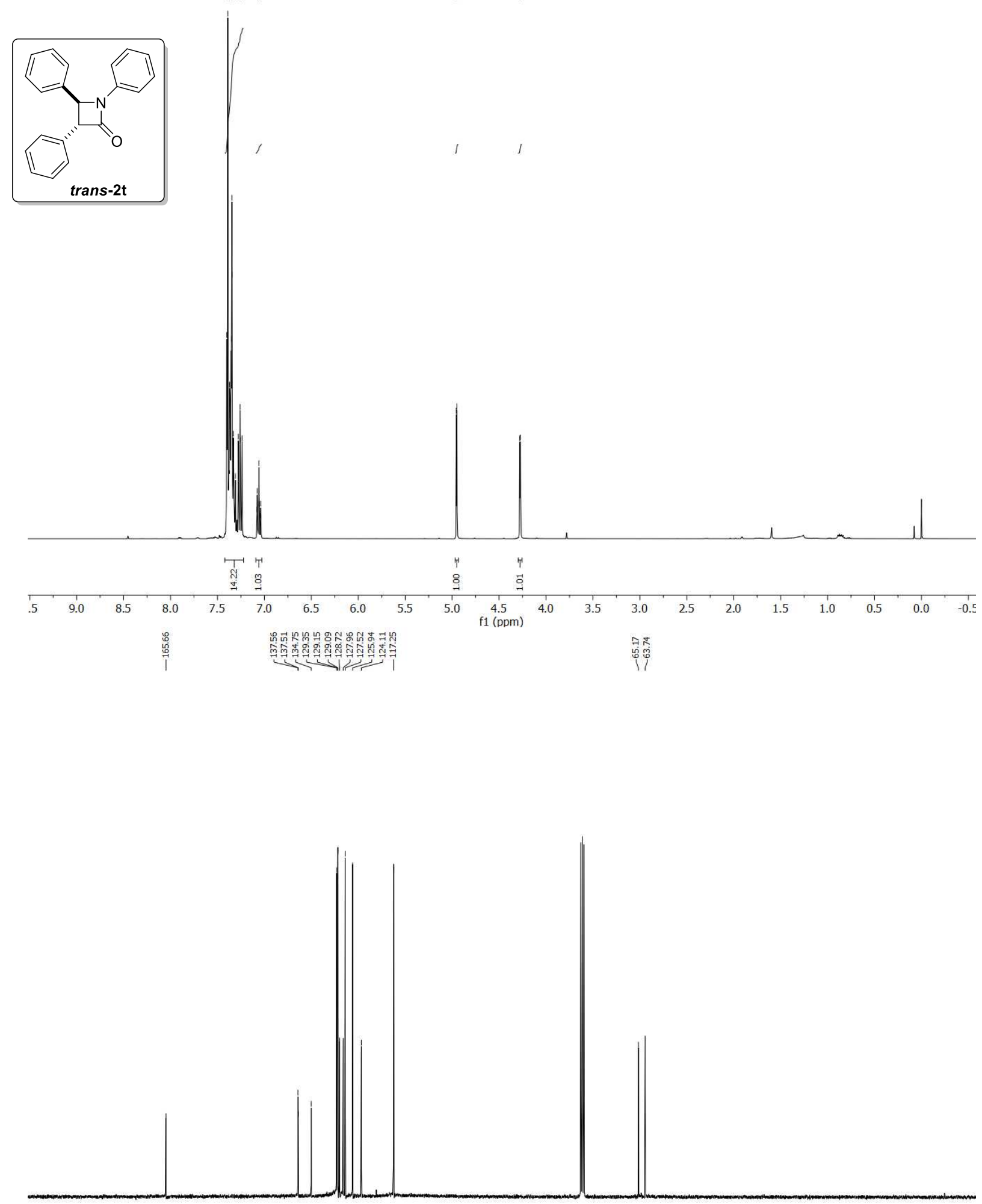

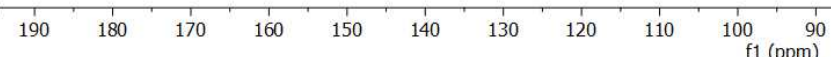




\section{References}

(1) Śnieżek, M.; Stecko, S.; Panfil, I.; Furman, B.; Chmielewski, M. J. Org. Chem. 2013, 78, 7048.

(2) Tian, Z.; Xu, J.; Liu, B.; Tan, Q.; Xu, B. Org. Lett. 2018, 20, 2603.

(3) (a) Bandar, J. S.; Sauer, G. S.; Wulff, W. D.; Lambert, T. H.; Vetticatt, M. J. J. Am. Chem. Soc. 2014, 136, 10700. (b) Murahashi, S.; Mitsui, H.; Shiota, T.; Tsuda, T.; Watanabe, S. J. Org. Chem. 1990, 55, 1736.

(4) Tyrrell, E.; Allen, J.; Jones, K.; Beauchet, R. Synthesis 2005, 2005, 2393.

(5) (a) Lyapkalo, I. M.; loffe, S. L.; Strelenko, Y. A.; Tartakovsky, V. A. Russ. Chem. Bull. 1996, 45, 856.

(b) Zelenin, K. N.; Motorina, I. A.; Sviridova, L. A.; Bezhan, I. P.; Ershov, A. Y.; Golubeva, G. A.; Bundel', Y. G. Chem. Heterocycl. Compd. 1987, 23, 1018.

(6) Wang, L.; Xie, X.; Liu, Y. Org. Lett. 2012, 14, 5848.

(7) Jiao, P.; Nakashima, D.; Yamamoto, H. Angew. Chem. Int. Ed. 2008, 47, 2411.

(8) Luo, Q.; Cao, C.-T.; Cao, Z.; Cao, C. J. Phys. Org. Chem. 2016, 29, 406.

(9) Lo, M. M. C.; Fu, G. C. J. Am. Chem. Soc. 2002, 124, 4572.

(10) Duguet, N.; Slawin, A. M. Z.; Smith, A. D. Org. Lett. 2009, 11, 3858.

(11) Ram, R. N.; Soni, V. K. J. Org. Chem. 2013, 78, 11935.

(12) Dooley, B. M.; Bowles, S. E.; Storr, T.; Frank, N. L. Org. Lett. 2007, 9, 4781.

(13) Singh, G.; Ishar, M. P. S.; Girdhar, N. K.; Singh, L. J. Heterocycl. Chem. 2005, 42, 1047.

(14) Ballard, N.; Aguirre, M.; Simula, A.; Agirre, A.; Leiza, J. R.; Asua, J. M.; van Es, S. ACS Macro Letters 2016, 5, 1019.

(15) Tang, S.; He, J.; Sun, Y.; He, L.; She, X. J. Org. Chem. 2010, 75, 1961.

(16) Otto, H. H.; Mayrhofer, R.; Bergmann, H. J. Liebigs Ann. 1983, 1983, 1152.

(17) Kim, B. J.; Park, Y. S.; Beak, P. J. Org. Chem. 1999, 64, 1705.

(18) Elriati, A.; Loose, J.; Mayrhofer, R.; Bergmann, H.-J.; Otto, H.-H. Monatsh. Chem. 2008, 139, 835.

(19) Pfleger, R.; Jäger, A. Chem. Ber. 1957, 90, 2460.

(20) Goyal, S.; Thakur, A.; Sharma, R.; Gangar, M.; Patel, B.; Nair, V. A. Asian J. Org. Chem. 2016, 5, 1359.

(21) Karageorgis, G.; Warriner, S.; Nelson, A. Nat. Chem. 2014, 6, 872.

(22) Kern, N.; Hoffmann, M.; Weibel, J.-M.; Pale, P.; Blanc, A. Tetrahedron 2014, 70, 5519.

(23) Kano, S.; Shibuya, S.; Ebata, T. J. Chem. Soc., Perkin Trans. 1 1982, 257.

(24) McKay, C. S.; Kennedy, D. C.; Pezacki, J. P. Tetrahedron Lett. 2009, 50, 1893. 